\title{
Partition Coefficients Involving Fluorous
}

Solvents

\author{
J. A. Gladysz, Charlotte Emnet, and József Rábai
}

6.1

Introduction

Partition coefficients quantify the equilibrium distribution of a solute between two immiscible phases, which are most often but not necessarily liquids. They see extensive use throughout chemistry, and their thermodynamic nuances have been analyzed in detail [1].

In order to rationally extract non-fluorous products from reactions involving fluorous solvents, partition coefficients for fluorous/non-fluorous liquid/liquid biphase systems are necessary. The design and optimization of fluorous catalysts and reagents require analogous data. Such partition coefficients constitute a direct measure of fluorophilicity, a term that is used interchangeably with fluorous phase affinity.

By 1999, only a few partition coefficients involving fluorous and organic phases had been measured $[2,3]$. Now there is a wealth of data, to which sophisticated analysis and parameterization methods have been applied [4-6]. The primary aim of this chapter is to summarize the literature reports, and qualitatively interpret the principal trends. Representative procedures for the determination of partition coefficients are also given.

\section{2}

\section{Literature Data}

Some investigators prefer to express partition coefficients as ratios that have been normalized to 100 (e.g., 98.3:1.7), others as ratios with either the less populated phase or the nonfluorous phase set to 1 (e.g., 57.8:1), and still others as logarithmic values. The abbreviation $P$ indicates a concentration ratio with the non-fluorous phase in the denominator. The natural logarithm of the $\mathrm{CF}_{3} \mathrm{C}_{6} \mathrm{~F}_{11} /$ toluene concentration ratio, $\ln \left\{\left[c\left(\mathrm{CF}_{3} \mathrm{C}_{6} \mathrm{~F}_{11}\right)\right] /[c\right.$ (toluene $\left.\left.)\right]\right\}$, has been given the abbreviation $f$, for fluorophilicity [4].

All partition coefficients measured in the authors' laboratories can be found in Table 6-1. Most of these involve the solvent system $\mathrm{CF}_{3} \mathrm{C}_{6} \mathrm{~F}_{11} /$ toluene. Many data from other research groups are also included. However, there are undoubtedly inadvertent omissions, for which the authors express their regrets. Since the entries are sorted by functional group, the placement of difunctional molecules is arbitrary. At the end of some sections, cross refer-

Handbook of Fluorous Chemistry. Edited by John A. Gladysz, Dennis P. Curran, István T. Horvath Copyright (C) 2004 WILEY-VCH Verlag GmbH \& Co. KGaA, Weinheim ISBN: 3-527-30617-X 
Tab. 6-1. Fluorous/organic liquid/liquid partition coefficients ${ }^{a}$

\begin{tabular}{|c|c|c|c|c|c|}
\hline Entry & Solute ${ }^{\mathrm{b}}$ & Solvent system & $\begin{array}{l}\text { Partitioning } \\
\text { fluorous: } \\
\text { organic (P) }\end{array}$ & Method & Ref. \\
\hline I & Alkanes & & & & \\
\hline I-1 & $\mathrm{CH}_{3}\left(\mathrm{CH}_{2}\right)_{8} \mathrm{CH}_{3}$ & $\mathrm{CF}_{3} \mathrm{C}_{6} \mathrm{~F}_{11}: \mathrm{CH}_{3} \mathrm{C}_{6} \mathrm{H}_{5}$ & $\begin{array}{l}\text { 5.4:94.6 } \\
(P=0.057)\end{array}$ & GLC & 1 \\
\hline $\mathrm{I}-2$ & $\mathrm{CH}_{3}\left(\mathrm{CH}_{2}\right)_{9} \mathrm{CH}_{3}$ & $\mathrm{CF}_{3} \mathrm{C}_{6} \mathrm{~F}_{11}: \mathrm{CH}_{3} \mathrm{C}_{6} \mathrm{H}_{5}$ & $\begin{array}{l}\text { 4.2:95.8 } \\
(P=0.044)\end{array}$ & GLC & 1 \\
\hline $\mathrm{I}-3$ & $\mathrm{CH}_{3}\left(\mathrm{CH}_{2}\right)_{10} \mathrm{CH}_{3}$ & $\mathrm{CF}_{3} \mathrm{C}_{6} \mathrm{~F}_{11}: \mathrm{CH}_{3} \mathrm{C}_{6} \mathrm{H}_{5}$ & $\begin{array}{l}\text { 3.4:96.6 } \\
(P=0.035)\end{array}$ & GLC & 1,2 \\
\hline $\mathrm{I}-4$ & $\mathrm{CH}_{3}\left(\mathrm{CH}_{2}\right)_{11} \mathrm{CH}_{3}$ & $\mathrm{CF}_{3} \mathrm{C}_{6} \mathrm{~F}_{11}: \mathrm{CH}_{3} \mathrm{C}_{6} \mathrm{H}_{5}$ & $\begin{array}{l}2.4: 97.6 \\
(P=0.025)\end{array}$ & GLC & 1 \\
\hline I-5 & $\mathrm{CH}_{3}\left(\mathrm{CH}_{2}\right)_{12} \mathrm{CH}_{3}$ & $\mathrm{CF}_{3} \mathrm{C}_{6} \mathrm{~F}_{11}: \mathrm{CH}_{3} \mathrm{C}_{6} \mathrm{H}_{5}$ & $\begin{array}{l}\text { 1.9:98.1 } \\
(P=0.019)\end{array}$ & GLC & 1 \\
\hline I-6 & $\mathrm{CH}_{3}\left(\mathrm{CH}_{2}\right)_{14} \mathrm{CH}_{3}$ & $\mathrm{CF}_{3} \mathrm{C}_{6} \mathrm{~F}_{11}: \mathrm{CH}_{3} \mathrm{C}_{6} \mathrm{H}_{5}$ & $\begin{array}{l}\text { 1.1:98.9 } \\
(P=0.011)\end{array}$ & GLC & 1 \\
\hline II & Alkenes & & & & \\
\hline II-1 & $\mathrm{CH}_{3}\left(\mathrm{CH}_{2}\right)_{7} \mathrm{CH}=\mathrm{CH}_{2}$ & $\mathrm{CF}_{3} \mathrm{C}_{6} \mathrm{~F}_{11}: \mathrm{CH}_{3} \mathrm{C}_{6} \mathrm{H}_{5}$ & $\begin{array}{l}4.8: 95.2 \\
(P=0.050)\end{array}$ & GLC & 3 \\
\hline II-2 & $\mathrm{CH}_{3}\left(\mathrm{CH}_{2}\right)_{8} \mathrm{CH}=\mathrm{CH}_{2}$ & $\mathrm{CF}_{3} \mathrm{C}_{6} \mathrm{~F}_{11}: \mathrm{CH}_{3} \mathrm{C}_{6} \mathrm{H}_{5}$ & $\begin{array}{l}3.7: 96.3 \\
(P=0.038)\end{array}$ & GLC & 3 \\
\hline II-3 & $\mathrm{CH}_{3}\left(\mathrm{CH}_{2}\right)_{9} \mathrm{CH}=\mathrm{CH}_{2}$ & $\mathrm{CF}_{3} \mathrm{C}_{6} \mathrm{~F}_{11}: \mathrm{CH}_{3} \mathrm{C}_{6} \mathrm{H}_{5}$ & $\begin{array}{l}2.5: 97.5 \\
(P=0.026)\end{array}$ & GLC & 2 \\
\hline II-4 & $\mathrm{CH}_{3}\left(\mathrm{CH}_{2}\right)_{10} \mathrm{CH}=\mathrm{CH}_{2}$ & $\mathrm{CF}_{3} \mathrm{C}_{6} \mathrm{~F}_{11}: \mathrm{CH}_{3} \mathrm{C}_{6} \mathrm{H}_{5}$ & $\begin{array}{l}\text { 1.9:98.1 } \\
(P=0.019)\end{array}$ & GLC & 3 \\
\hline II-5 & $\mathrm{CH}_{3}\left(\mathrm{CH}_{2}\right)_{11} \mathrm{CH}=\mathrm{CH}_{2}$ & $\mathrm{CF}_{3} \mathrm{C}_{6} \mathrm{~F}_{11}: \mathrm{CH}_{3} \mathrm{C}_{6} \mathrm{H}_{5}$ & $\begin{array}{l}\text { 1.6:98.4 } \\
(P=0.016)\end{array}$ & GLC & 3 \\
\hline II-6 & $\mathrm{CH}_{3}\left(\mathrm{CH}_{2}\right)_{13} \mathrm{CH}=\mathrm{CH}_{2}$ & $\mathrm{CF}_{3} \mathrm{C}_{6} \mathrm{~F}_{11}: \mathrm{CH}_{3} \mathrm{C}_{6} \mathrm{H}_{5}$ & $\begin{array}{l}\text { 0.9:99.1 } \\
(P=0.009)\end{array}$ & GLC & 3 \\
\hline II-7c & $\mathrm{R}_{\mathrm{f} 8} \mathrm{CH}=\mathrm{CH}_{2}$ & $\mathrm{CF}_{3} \mathrm{C}_{6} \mathrm{~F}_{11}: \mathrm{CH}_{3} \mathrm{C}_{6} \mathrm{H}_{5}$ & $\begin{array}{l}93.5: 6.5 \\
(P=14.4)^{\mathrm{d}}\end{array}$ & GLC & $4 a$ \\
\hline II--8 & $E-\mathrm{R}_{\mathrm{f} 6} \mathrm{CH}=\mathrm{CHC}_{6} \mathrm{H}_{13}$ & $\mathrm{C}_{10} \mathrm{~F}_{18} \mathrm{e}^{\mathrm{e}} \mathrm{C}_{16} \mathrm{H}_{34}$ & $\begin{array}{l}\text { 63.4:36.6 } \\
(P=1.73)\end{array}$ & GLC & 5 \\
\hline II-9c & & $\left(\mathrm{C}_{4} \mathrm{~F}_{9} \mathrm{CH}=\right)_{2}: \mathrm{C}_{16} \mathrm{H}_{34}$ & $\begin{array}{l}72.5: 27.5 \\
(P=2.63)\end{array}$ & GLC & 5 \\
\hline $\mathrm{II}-10^{\mathrm{c}}$ & & $\mathrm{C}_{8} \mathrm{~F}_{17} \mathrm{Br}: \mathrm{C}_{16} \mathrm{H}_{34}$ & $\begin{array}{l}74.9: 25.1 \\
(P=2.98)\end{array}$ & GLC & 5 \\
\hline II-11 ${ }^{\mathrm{c}}$ & $Z-\mathrm{R}_{\mathrm{f} 6} \mathrm{CH}=\mathrm{CHC}_{6} \mathrm{H}_{13}$ & $\mathrm{C}_{10} \mathrm{~F}_{18}: \mathrm{C}_{16} \mathrm{H}_{34}$ & $\begin{array}{l}\text { 62.1:37.9 } \\
(P=1.64)\end{array}$ & GLC & 5 \\
\hline $\mathrm{II}-12^{\mathrm{c}}$ & & $\left(\mathrm{C}_{4} \mathrm{~F}_{9} \mathrm{CH}=\right)_{2}: \mathrm{C}_{16} \mathrm{H}_{34}$ & $\begin{array}{l}67.5: 32.5 \\
(P=2.08)\end{array}$ & GLC & 5 \\
\hline $\mathrm{II}-13^{\mathrm{c}}$ & & $\mathrm{C}_{8} \mathrm{~F}_{17} \mathrm{Br}: \mathrm{C}_{16} \mathrm{H}_{34}$ & $\begin{array}{l}72.6: 27.4 \\
(P=2.65)\end{array}$ & GLC & 5 \\
\hline II-14 & $E-\mathrm{R}_{\mathrm{f}} \mathrm{CH}=\mathrm{CHC}_{8} \mathrm{H}_{17}$ & $\mathrm{C}_{10} \mathrm{~F}_{18} \mathrm{e}^{\mathrm{e}} \mathrm{C}_{16} \mathrm{H}_{34}$ & $\begin{array}{l}42: 58 \\
(P=0.72)\end{array}$ & GLC & 5 \\
\hline $\mathrm{II}-15^{\mathrm{c}}$ & & $\left(\mathrm{C}_{4} \mathrm{~F}_{9} \mathrm{CH}=\right)_{2}: \mathrm{C}_{16} \mathrm{H}_{34}$ & $\begin{array}{l}49: 51 \\
(P=0.95)\end{array}$ & GLC & 5 \\
\hline II-16c & & $\mathrm{C}_{8} \mathrm{~F}_{17} \mathrm{Br}: \mathrm{C}_{16} \mathrm{H}_{34}$ & $\begin{array}{l}53.1: 46.9 \\
(P=1.13)\end{array}$ & GLC & 5 \\
\hline
\end{tabular}


$58 \mid 6$ Partition Coefficients Involving Fluorous Solvents

Tab. 6-1. (continued)

\begin{tabular}{|c|c|c|c|c|c|}
\hline Entry & Solute & Solvent system & $\begin{array}{l}\text { Partitioning } \\
\text { fluorous: } \\
\text { organic }(P)\end{array}$ & Method & Ref. \\
\hline II-17c & $Z-\mathrm{R}_{\mathrm{f} 6} \mathrm{CH}=\mathrm{CHC}_{8} \mathrm{H}_{17}$ & $\mathrm{C}_{10} \mathrm{~F}_{18} \mathrm{e}: \mathrm{C}_{16} \mathrm{H}_{34}$ & $\begin{array}{l}50.0: 50.0 \\
(P=1.00)\end{array}$ & GLC & 5 \\
\hline II-18c & & $\left(\mathrm{C}_{4} \mathrm{~F}_{9} \mathrm{CH}=\right)_{2}: \mathrm{C}_{16} \mathrm{H}_{34}$ & $\begin{array}{l}53.1: 46.9 \\
(P=1.13)\end{array}$ & GLC & 5 \\
\hline $\mathrm{II}-19^{\mathrm{c}}$ & & $\mathrm{C}_{8} \mathrm{~F}_{17} \mathrm{Br}: \mathrm{C}_{16} \mathrm{H}_{34}$ & $\begin{array}{l}60.0: 40.0 \\
(P=1.50)\end{array}$ & GLC & 5 \\
\hline II-20 & $E-\mathrm{R}_{\mathrm{f} 6} \mathrm{CH}=\mathrm{CHC}_{10} \mathrm{H}_{21}$ & $\mathrm{C}_{10} \mathrm{~F}_{18} \mathrm{e}^{\mathrm{e}} \mathrm{C}_{16} \mathrm{H}_{34}$ & $\begin{array}{l}20: 80 \\
(P=0.25)\end{array}$ & GLC & 5 \\
\hline II-21 ${ }^{\mathrm{c}}$ & & $\left(\mathrm{C}_{4} \mathrm{~F}_{9} \mathrm{CH}=\right)_{2}: \mathrm{C}_{16} \mathrm{H}_{34}$ & $\begin{array}{l}43: 57 \\
(P=0.74)\end{array}$ & GLC & 5 \\
\hline II-22c & & $\mathrm{C}_{8} \mathrm{~F}_{17} \mathrm{Br}: \mathrm{C}_{16} \mathrm{H}_{34}$ & $\begin{array}{l}44: 56 \\
(P=0.80)\end{array}$ & GLC & 5 \\
\hline II-23c & $Z-\mathrm{R}_{\mathrm{f} 6} \mathrm{CH}=\mathrm{CHC}_{10} \mathrm{H}_{21}$ & $\mathrm{C}_{10} \mathrm{~F}_{18} \mathrm{e}: \mathrm{C}_{16} \mathrm{H}_{34}$ & $\begin{array}{l}28: 72 \\
(P=0.38)\end{array}$ & GLC & 5 \\
\hline II-24c & & $\left(\mathrm{C}_{4} \mathrm{~F}_{9} \mathrm{CH}=\right)_{2}: \mathrm{C}_{16} \mathrm{H}_{34}$ & $\begin{array}{l}40: 60 \\
(P=0.68)\end{array}$ & GLC & 5 \\
\hline II-25c & & $\mathrm{C}_{8} \mathrm{~F}_{17} \mathrm{Br}: \mathrm{C}_{16} \mathrm{H}_{34}$ & $\begin{array}{l}47: 53 \\
(P=0.88)\end{array}$ & GLC & 5 \\
\hline II-26c & $E-\mathrm{R}_{\mathrm{f} 8} \mathrm{CH}=\mathrm{CHC}_{6} \mathrm{H}_{13}$ & $\mathrm{C}_{10} \mathrm{~F}_{18} \mathrm{e}^{\mathrm{e}} \mathrm{C}_{16} \mathrm{H}_{34}$ & $\begin{array}{l}79.8: 20.2 \\
(\boldsymbol{P}=3.95)\end{array}$ & GLC & 5 \\
\hline II-27c & & $\left(\mathrm{C}_{4} \mathrm{~F}_{9} \mathrm{CH}=\right)_{2}: \mathrm{C}_{16} \mathrm{H}_{34}$ & $\begin{array}{l}86.0: 14.0 \\
(P=6.13)\end{array}$ & GLC & 5 \\
\hline II-28c & & $\mathrm{C}_{8} \mathrm{~F}_{17} \mathrm{Br}: \mathrm{C}_{16} \mathrm{H}_{34}$ & $\begin{array}{l}86.4: 13.6 \\
(P=6.38)\end{array}$ & GLC & 5 \\
\hline II-29c & $Z-\mathrm{R}_{\mathrm{f} 8} \mathrm{CH}=\mathrm{CHC}_{6} \mathrm{H}_{13}$ & $\mathrm{C}_{10} \mathrm{~F}_{18} \mathrm{e}: \mathrm{C}_{16} \mathrm{H}_{34}$ & $\begin{array}{l}77.2: 22.8 \\
(P=3.38)\end{array}$ & GLC & 5 \\
\hline $\mathrm{II}-30^{\mathrm{c}}$ & & $\left(\mathrm{C}_{4} \mathrm{~F}_{9} \mathrm{CH}=\right)_{2}: \mathrm{C}_{16} \mathrm{H}_{34}$ & $\begin{array}{l}82.0: 18.0 \\
(P=4.55)\end{array}$ & GLC & 5 \\
\hline II-31 ${ }^{\mathrm{c}}$ & & $\mathrm{C}_{8} \mathrm{~F}_{17} \mathrm{Br}: \mathrm{C}_{16} \mathrm{H}_{34}$ & $\begin{array}{l}83.6: 16.4 \\
(P=5.11)\end{array}$ & GLC & 5 \\
\hline $\mathrm{II}-32^{\mathrm{c}}$ & $E-\mathrm{R}_{\mathrm{f} 8} \mathrm{CH}=\mathrm{CHC}_{8} \mathrm{H}_{17}$ & $\mathrm{C}_{10} \mathrm{~F}_{18} \mathrm{e}^{\mathrm{e}} \mathrm{C}_{16} \mathrm{H}_{34}$ & $\begin{array}{l}72.5: 27.5 \\
(P=2.63)\end{array}$ & GLC & 5 \\
\hline II-33c & & $\left(\mathrm{C}_{4} \mathrm{~F}_{9} \mathrm{CH}=\right)_{2}: \mathrm{C}_{16} \mathrm{H}_{34}$ & $\begin{array}{l}76.7: 23.3 \\
(P=3.30)\end{array}$ & GLC & 5 \\
\hline II-34 & & $\mathrm{C}_{8} \mathrm{~F}_{17} \mathrm{Br}: \mathrm{C}_{16} \mathrm{H}_{34}$ & $\begin{array}{l}79.2: 20.8 \\
(P=3.81)\end{array}$ & GLC & 5 \\
\hline II-35 & $Z-\mathrm{R}_{\mathrm{f} 8} \mathrm{CH}=\mathrm{CHC}_{8} \mathrm{H}_{17}$ & $\mathrm{C}_{10} \mathrm{~F}_{18} \mathrm{e}: \mathrm{C}_{16} \mathrm{H}_{34}$ & $\begin{array}{l}71.5: 28.5 \\
(P=2.51)\end{array}$ & GLC & 5 \\
\hline II-36c & & $\left(\mathrm{C}_{4} \mathrm{~F}_{9} \mathrm{CH}=\right)_{2}: \mathrm{C}_{16} \mathrm{H}_{34}$ & $\begin{array}{l}76.0: 24.0 \\
(P=3.17)\end{array}$ & GLC & 5 \\
\hline II-37c & & $\mathrm{C}_{8} \mathrm{~F}_{17} \mathrm{Br}: \mathrm{C}_{16} \mathrm{H}_{34}$ & $\begin{array}{l}78.6: 21.4 \\
(P=3.67)\end{array}$ & GLC & 5 \\
\hline II-38 & $E-\mathrm{R}_{\mathrm{f} 8} \mathrm{CH}=\mathrm{CHC}_{10} \mathrm{H}_{21}$ & $\mathrm{C}_{10} \mathrm{~F}_{18} \mathrm{e}^{\mathrm{e}} \mathrm{C}_{16} \mathrm{H}_{34}$ & $\begin{array}{l}54.8: 45.2 \\
(P=1.21)\end{array}$ & GLC & 5 \\
\hline II-39c & & $\left(\mathrm{C}_{4} \mathrm{~F}_{9} \mathrm{CH}=\right)_{2}: \mathrm{C}_{16} \mathrm{H}_{34}$ & $\begin{array}{l}63.1: 36.9 \\
(P=1.71)\end{array}$ & GLC & 5 \\
\hline
\end{tabular}


Tab. 6-1. (continued)

\begin{tabular}{|c|c|c|c|c|c|}
\hline Entry & Solute $^{\mathrm{b}}$ & Solvent system & $\begin{array}{l}\text { Partitioning } \\
\text { fluorous: } \\
\text { organic (P) }\end{array}$ & Method & Ref. \\
\hline II $-40^{c}$ & & $\mathrm{C}_{8} \mathrm{~F}_{17} \mathrm{Br}: \mathrm{C}_{16} \mathrm{H}_{34}$ & $\begin{array}{l}70.8: 29.2 \\
(P=2.43)\end{array}$ & GLC & 5 \\
\hline II-41c & $Z-\mathrm{R}_{\mathrm{f} 8} \mathrm{CH}=\mathrm{CHC}_{10} \mathrm{H}_{21}$ & $\mathrm{C}_{10} \mathrm{~F}_{18}{ }^{\mathrm{e}}: \mathrm{C}_{16} \mathrm{H}_{34}$ & $\begin{array}{l}54.1: 45.9 \\
(P=1.18)\end{array}$ & GLC & 5 \\
\hline II $-42^{c}$ & & $\left(\mathrm{C}_{4} \mathrm{~F}_{9} \mathrm{CH}=\right)_{2}: \mathrm{C}_{16} \mathrm{H}_{34}$ & $\begin{array}{l}56.7: 43.3 \\
(P=1.31)\end{array}$ & GLC & 5 \\
\hline II $-43^{c}$ & & $\mathrm{C}_{8} \mathrm{~F}_{17} \mathrm{Br}: \mathrm{C}_{16} \mathrm{H}_{34}$ & $\begin{array}{l}\text { 65.5:34.5 } \\
(P=1.90)\end{array}$ & GLC & 5 \\
\hline II $-44^{c}$ & $c-\mathrm{C}_{5} \mathrm{H}_{4}\left[\left(\mathrm{CH}_{2}\right)_{2} \mathrm{R}_{\mathrm{f} 4}\right]_{2}{ }^{\mathrm{f}}$ & $\mathrm{CF}_{3} \mathrm{C}_{6} \mathrm{~F}_{11}: \mathrm{CH}_{3} \mathrm{C}_{6} \mathrm{H}_{5}$ & $\begin{array}{l}55: 45 \\
(P=1.2)\end{array}$ & GLC & 6 \\
\hline II $-45^{c}$ & $c-\mathrm{C}_{5} \mathrm{H}_{4}\left[\left(\mathrm{CH}_{2}\right)_{2} \mathrm{R}_{\mathrm{f} 6}\right]_{2}{ }^{\mathrm{f}}$ & $\mathrm{CF}_{3} \mathrm{C}_{6} \mathrm{~F}_{11}: \mathrm{CH}_{3} \mathrm{C}_{6} \mathrm{H}_{5}$ & $\begin{array}{l}83: 17 \\
(P=4.9)\end{array}$ & GLC & 6 \\
\hline II-46 & $c-\mathrm{C}_{5} \mathrm{H}_{4}\left[\left(\mathrm{CH}_{2}\right)_{2} \mathrm{R}_{\mathrm{f} 8}\right]_{2}{ }^{\mathrm{f}}$ & $\mathrm{CF}_{3} \mathrm{C}_{6} \mathrm{~F}_{11}: \mathrm{CH}_{3} \mathrm{C}_{6} \mathrm{H}_{5}$ & $\begin{array}{l}90: 10 \\
(P=8.8)\end{array}$ & GLC & 6 \\
\hline II-47c & $c-\mathrm{C}_{5} \mathrm{H}_{4}\left[\left(\mathrm{CH}_{2}\right)_{2} \mathrm{R}_{\mathrm{f} 4}\right]\left[\left(\mathrm{CH}_{2}\right)_{2} \mathrm{R}_{\mathrm{f} 6}\right]^{\mathrm{f}}$ & $\mathrm{CF}_{3} \mathrm{C}_{6} \mathrm{~F}_{11}: \mathrm{CH}_{3} \mathrm{C}_{6} \mathrm{H}_{5}$ & $\begin{array}{l}75: 25 \\
(P=3.0)\end{array}$ & GLC & 6 \\
\hline II $-48^{c}$ & $c-\mathrm{C}_{5} \mathrm{H}_{4}\left[\left(\mathrm{CH}_{2}\right)_{2} \mathrm{R}_{\mathrm{f} 4}\right]\left[\left(\mathrm{CH}_{2}\right)_{2} \mathrm{R}_{\mathrm{f} 8}\right]^{\mathrm{f}}$ & $\mathrm{CF}_{3} \mathrm{C}_{6} \mathrm{~F}_{11}: \mathrm{CH}_{3} \mathrm{C}_{6} \mathrm{H}_{5}$ & $\begin{array}{l}82: 18 \\
(P=4.6)\end{array}$ & GLC & 6 \\
\hline II $-49^{c}$ & $\begin{array}{l}c-\mathrm{C}_{5} \mathrm{H}_{4}\left[\left(\mathrm{CH}_{2}\right)_{2} \mathrm{R}_{\mathrm{f} 6}\right]\left[\left(\mathrm{CH}_{2}\right)_{2} \mathrm{R}_{\mathrm{f} 8}\right]^{\mathrm{f}} \\
\text { for further compounds see XII and XIII }\end{array}$ & $\mathrm{CF}_{3} \mathrm{C}_{6} \mathrm{~F}_{11}: \mathrm{CH}_{3} \mathrm{C}_{6} \mathrm{H}_{5}$ & $\begin{array}{l}86: 14 \\
(P=6.4)\end{array}$ & GLC & 6 \\
\hline III & Ketones and Aldehydes & & & & \\
\hline III-1 & cyclohexanone & $\mathrm{CF}_{3} \mathrm{C}_{6} \mathrm{~F}_{11}: \mathrm{CH}_{3} \mathrm{C}_{6} \mathrm{H}_{5}$ & $\begin{array}{l}2.2: 97.8 \\
(P=0.022)\end{array}$ & GLC & 2 \\
\hline III-2 & 2-cyclohexen-1-one & $\mathrm{CF}_{3} \mathrm{C}_{6} \mathrm{~F}_{11}: \mathrm{CH}_{3} \mathrm{C}_{6} \mathrm{H}_{5}$ & $\begin{array}{l}1.7: 98.3 \\
(P=0.017)\end{array}$ & GLC & 2 \\
\hline III-3 & $\mathrm{R}_{\mathrm{f} 8}\left(\mathrm{CH}_{2}\right)_{3}-\mathrm{C}(\mathrm{O})\left(\mathrm{CH}_{2}\right)_{2} \mathrm{R}_{\mathrm{f} 8}$ & $\mathrm{CF}_{3} \mathrm{C}_{6} \mathrm{~F}_{11}: \mathrm{CH}_{3} \mathrm{C}_{6} \mathrm{H}_{5}$ & $\begin{array}{l}84.6: 15.4 \\
(P=5.49)\end{array}$ & GLC & 7 \\
\hline & $\mathrm{R}_{\mathrm{f} 8}$ & & & & \\
\hline III- $4^{c}$ & $\mathrm{R}_{\mathrm{f} 8}=\mathrm{CHO}$ & $\mathrm{CF}_{3} \mathrm{C}_{6} \mathrm{~F}_{11}: \mathrm{CH}_{3} \mathrm{C}_{6} \mathrm{H}_{5}$ & $\begin{array}{l}98.6: 1.4 \\
(P=73.0)^{d}\end{array}$ & GLC & $4 \mathrm{a}$ \\
\hline IV & Alcohols & & & & \\
\hline IV-1 & cyclohexanol & $\mathrm{CF}_{3} \mathrm{C}_{6} \mathrm{~F}_{11}: \mathrm{CH}_{3} \mathrm{C}_{6} \mathrm{H}_{5}$ & $\begin{array}{l}\text { 1.6:98.4 } \\
(P=0.016)\end{array}$ & GLC & 2 \\
\hline IV-2c & $\mathrm{CF}_{3} \mathrm{CH}_{2} \mathrm{OH}$ & $\mathrm{CF}_{3} \mathrm{C}_{6} \mathrm{~F}_{11}: \mathrm{CH}_{3} \mathrm{C}_{6} \mathrm{H}_{5}$ & $\begin{array}{l}14.5: 85.5 \\
(P=0.170)^{d}\end{array}$ & GLC & 8 \\
\hline IV-3c & $\left(\mathrm{CF}_{3}\right)_{2} \mathrm{CHOH}$ & $\mathrm{CF}_{3} \mathrm{C}_{6} \mathrm{~F}_{11}: \mathrm{CH}_{3} \mathrm{C}_{6} \mathrm{H}_{5}$ & $\begin{array}{l}26.7: 73.3 \\
(P=0.364)^{d}\end{array}$ & GLC & 8 \\
\hline IV-4c & $\mathrm{R}_{\mathrm{f} 6}\left(\mathrm{CH}_{2}\right)_{2} \mathrm{OH}$ & $\mathrm{CF}_{3} \mathrm{C}_{6} \mathrm{~F}_{11}: \mathrm{CH}_{3} \mathrm{C}_{6} \mathrm{H}_{5}$ & $\begin{array}{l}52: 48 \\
(P=1.1)^{\mathrm{d}}\end{array}$ & GLC & 8 \\
\hline IV-5c & $\mathrm{R}_{\mathrm{f} 6}\left(\mathrm{CH}_{2}\right)_{3} \mathrm{OH}$ & $\mathrm{CF}_{3} \mathrm{C}_{6} \mathrm{~F}_{11}: \mathrm{CH}_{3} \mathrm{C}_{6} \mathrm{H}_{5}$ & $\begin{array}{l}44: 56 \\
(P=0.79)^{\mathrm{d}}\end{array}$ & GLC & 8 \\
\hline
\end{tabular}


$60 \mid 6$ Partition Coefficients Involving Fluorous Solvents

Tab. 6-1. (continued)

\begin{tabular}{|c|c|c|c|c|c|}
\hline Entry & Solute ${ }^{\mathrm{b}}$ & Solvent system & $\begin{array}{l}\text { Partitioning } \\
\text { fluorous: } \\
\text { organic (P) }\end{array}$ & Method & Ref. \\
\hline IV-6c & $\mathrm{R}_{\mathrm{f} 8}\left(\mathrm{CH}_{2}\right)_{2} \mathrm{OH}$ & $\mathrm{CF}_{3} \mathrm{C}_{6} \mathrm{~F}_{11}: \mathrm{CH}_{3} \mathrm{C}_{6} \mathrm{H}_{5}$ & $\begin{array}{l}73.5: 26.5 \\
(P=2.77)^{d}\end{array}$ & GLC & 8 \\
\hline IV-7c & $\mathrm{R}_{\mathrm{f8}}\left(\mathrm{CH}_{2}\right)_{3} \mathrm{OH}$ & $\mathrm{CF}_{3} \mathrm{C}_{6} \mathrm{~F}_{11}: \mathrm{CH}_{3} \mathrm{C}_{6} \mathrm{H}_{5}$ & $\begin{array}{l}\text { 64:36 } \\
(P=1.8)^{\mathrm{d}}\end{array}$ & GLC & 8 \\
\hline IV-8c & $\mathrm{R}_{\mathrm{f} 10}\left(\mathrm{CH}_{2}\right)_{3} \mathrm{OH}$ & $\mathrm{CF}_{3} \mathrm{C}_{6} \mathrm{~F}_{11}: \mathrm{CH}_{3} \mathrm{C}_{6} \mathrm{H}_{5}$ & $\begin{array}{l}80.5: 19.5 \\
(P=4.14)^{d}\end{array}$ & GLC & 8 \\
\hline IV-9g & {$\left[\mathrm{R}_{\mathrm{f} 6}\left(\mathrm{CH}_{2}\right)_{2}\right]_{3} \mathrm{COH}$} & $\mathrm{C}_{6} \mathrm{~F}_{14}: \mathrm{THF}$ & $\begin{array}{l}90.9: 9.1 \\
(P=10.0)\end{array}$ & - & 9 \\
\hline IV-10 ${ }^{\mathrm{h}}$ & & $\mathrm{C}_{6} \mathrm{~F}_{14}: \mathrm{THF}$ & $\begin{array}{l}95.8: 4.2 \\
(\boldsymbol{P}=23.0)\end{array}$ & - & 9 \\
\hline & $\mathrm{R}_{\mathrm{f8}}$ & & & & \\
\hline IV-11 ${ }^{c}$ & $\mathrm{R}_{\mathrm{f8}}$ & $\mathrm{CF}_{3} \mathrm{C}_{6} \mathrm{~F}_{11}: \mathrm{CH}_{3} \mathrm{C}_{6} \mathrm{H}_{5}$ & $\begin{array}{l}97.4: 2.6 \\
(P=38.1)^{\mathrm{d}}\end{array}$ & GLC & $4 a$ \\
\hline IV-12g & {$\left[\mathrm{R}_{\mathrm{f} 6}\left(\mathrm{CH}_{2}\right)_{2}\right]_{2} \mathrm{C}\left(\mathrm{OCH}_{3}\right) \mathrm{CH}(\mathrm{OH}) \mathrm{C}_{6} \mathrm{H}_{5}$} & $\mathrm{C}_{6} \mathrm{~F}_{14}: \mathrm{THF}$ & $\begin{array}{l}19: 81 \\
(P=0.24)\end{array}$ & - & 9 \\
\hline IV-13g & $\underset{\mathrm{HO}}{\mathrm{C}_{6} \mathrm{H}_{5}}$ & $\mathrm{C}_{6} \mathrm{~F}_{14}: \mathrm{CH}_{3} \mathrm{CN}$ & $\begin{array}{l}88: 12 \\
(P=7.3)\end{array}$ & gravimet & 10 \\
\hline IV-14g & & $\mathrm{C}_{6} \mathrm{~F}_{14}: \mathrm{CH}_{3} \mathrm{C}_{6} \mathrm{H}_{5}$ & $\begin{array}{l}59: 41 \\
(P=1.4)\end{array}$ & gravimet & 10 \\
\hline IV-15g & & $\mathrm{C}_{6} \mathrm{~F}_{14}: \mathrm{CH}_{2} \mathrm{Cl}_{2}$ & $\begin{array}{l}38: 62 \\
(P=0.61)\end{array}$ & gravimet & 10 \\
\hline IV-16g & $\mathrm{HO}$ & $\mathrm{C}_{6} \mathrm{~F}_{14}: \mathrm{CH}_{3} \mathrm{CN}$ & $\begin{array}{l}82: 18 \\
(P=4.6)\end{array}$ & gravimet & 10 \\
\hline IV-17g & & $\mathrm{C}_{6} \mathrm{~F}_{14}: \mathrm{CH}_{3} \mathrm{C}_{6} \mathrm{H}_{5}$ & $\begin{array}{l}48: 52 \\
(P=0.92)\end{array}$ & gravimet & 10 \\
\hline IV-18g & & $\mathrm{C}_{6} \mathrm{~F}_{14}: \mathrm{CH}_{2} \mathrm{Cl}_{2}$ & $\begin{array}{l}25: 75 \\
(P=0.33)\end{array}$ & gravimet & 10 \\
\hline IV-19g & $\left(\mathrm{R}_{\mathrm{f} 6}\left(\mathrm{CH}_{2}\right)_{2}\right)_{3} \mathrm{C}^{-}$ & $\mathrm{C}_{6} \mathrm{~F}_{14}: \mathrm{CH}_{3} \mathrm{CN}$ & $\begin{array}{l}\mathbf{9 7 : 3} \\
(P=32)\end{array}$ & gravimet & 10 \\
\hline IV-20g & & $\mathrm{C}_{6} \mathrm{~F}_{14}: \mathrm{CH}_{3} \mathrm{C}_{6} \mathrm{H}_{5}$ & $\begin{array}{l}\mathbf{9 7 : 3} \\
(P=32)\end{array}$ & gravimet & 10 \\
\hline IV-219 & & $\mathrm{C}_{6} \mathrm{~F}_{14}: \mathrm{CH}_{2} \mathrm{Cl}_{2}$ & $\begin{array}{l}\mathbf{9 4 : 6} \\
(P=16)\end{array}$ & gravimet & 10 \\
\hline
\end{tabular}


6.2 Literature Data $\mid 61$

Tab. 6-1. (continued)

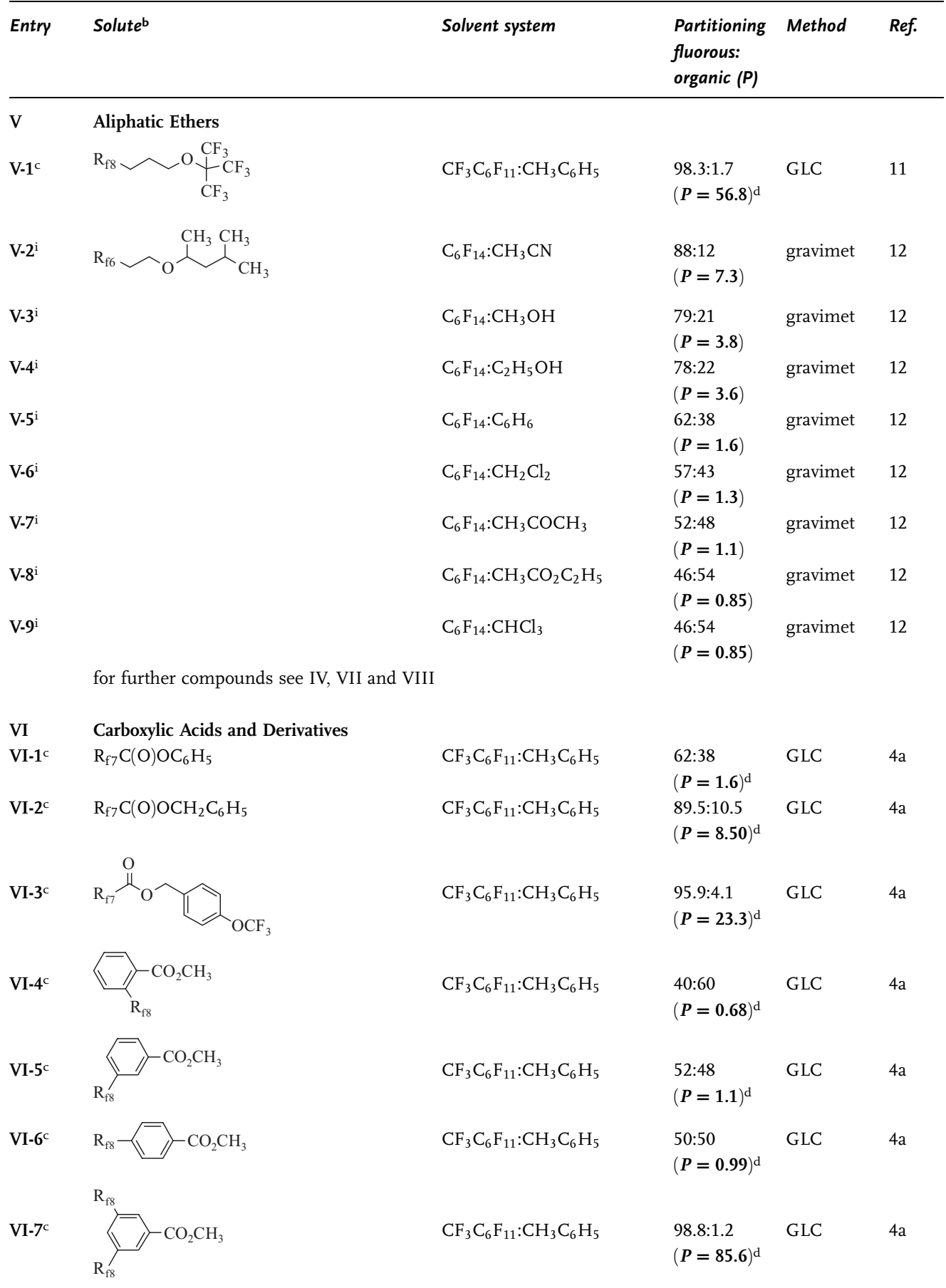


$62 \mid 6$ Partition Coefficients Involving Fluorous Solvents

Tab. 6-1. (continued)

\begin{tabular}{|c|c|c|c|c|c|}
\hline Entry & Solute & Solvent system & $\begin{array}{l}\text { Partitioning } \\
\text { fluorous: } \\
\text { organic (P) }\end{array}$ & Method & Ref. \\
\hline VI- $8^{c}$ & $\mathrm{CH}_{3} \mathrm{CH}_{2} \stackrel{\mathrm{O}}{\stackrel{\mathrm{O}}{\underset{\mathrm{O}}{\mathrm{C}}} \underset{\left(\mathrm{CH}_{2}\right)_{2} \mathrm{R}_{\mathrm{f} 8}}{\mathrm{CH}_{3}}\left(\mathrm{CH}_{2} \mathrm{R}_{\mathrm{f8}}\right.}$ & $\mathrm{C}_{6} \mathrm{~F}_{14}: c-\mathrm{C}_{6} \mathrm{H}_{12}$ & $\begin{array}{l}89.0: 11.0 \\
(P=8.10)\end{array}$ & gravimet & 13 \\
\hline VI-9c & & $\mathrm{CF}_{3} \mathrm{C}_{6} \mathrm{~F}_{11}: c-\mathrm{C}_{6} \mathrm{H}_{12}$ & $\begin{array}{l}90.5: 9.5 \\
(\boldsymbol{P}=9.58)\end{array}$ & gravimet & 13 \\
\hline VI-10c & & $\mathrm{C}_{6} \mathrm{~F}_{14}: \mathrm{CH}_{3} \mathrm{C}_{6} \mathrm{H}_{5}$ & $\begin{array}{l}88.6: 11.4 \\
(P=7.75)\end{array}$ & gravimet & 13 \\
\hline VI-11 ${ }^{c}$ & & $\mathrm{CF}_{3} \mathrm{C}_{6} \mathrm{~F}_{11}: \mathrm{CH}_{3} \mathrm{C}_{6} \mathrm{H}_{5}$ & $\begin{array}{l}86.6: 13.4 \\
(P=6.46)\end{array}$ & gravimet & 13 \\
\hline VI-12c & & $\mathrm{C}_{6} \mathrm{~F}_{14}: \mathrm{CH}_{2} \mathrm{Cl}_{2}$ & $\begin{array}{l}90.1: 9.9 \\
(P=9.08)\end{array}$ & gravimet & 13 \\
\hline VI-13c & & $\mathrm{CF}_{3} \mathrm{C}_{6} \mathrm{~F}_{11}: \mathrm{CH}_{2} \mathrm{Cl}_{2}$ & $\begin{array}{l}93.8: 6.2 \\
(P=15.4)\end{array}$ & gravimet & 13 \\
\hline VI-14c & & $\mathrm{C}_{6} \mathrm{~F}_{14}: \mathrm{CHCl}_{3}$ & $\begin{array}{l}75.4: 24.6 \\
(P=3.06)\end{array}$ & gravimet & 13 \\
\hline VI-15c & & $\mathrm{CF}_{3} \mathrm{C}_{6} \mathrm{~F}_{11}: \mathrm{CHCl}_{3}$ & $\begin{array}{l}85.6: 14.4 \\
(P=5.93)\end{array}$ & gravimet & 13 \\
\hline VI-16c & & $\mathrm{C}_{6} \mathrm{~F}_{14}: \mathrm{CH}_{3} \mathrm{CO}_{2} \mathrm{C}_{2} \mathrm{H}_{5}$ & $\begin{array}{l}72.1: 27.9 \\
(P=2.59)\end{array}$ & gravimet & 13 \\
\hline VI-17c & & $\mathrm{CF}_{3} \mathrm{C}_{6} \mathrm{~F}_{11}: \mathrm{CH}_{3} \mathrm{CO}_{2} \mathrm{C}_{2} \mathrm{H}_{5}$ & $\begin{array}{l}76.5: 23.5 \\
(P=3.25)\end{array}$ & gravimet & 13 \\
\hline VI-18c & & $\mathrm{C}_{6} \mathrm{~F}_{14}: \mathrm{THF}$ & $\begin{array}{l}73.0: 27.0 \\
(P=2.70)\end{array}$ & gravimet & 13 \\
\hline VI-19c & & $\mathrm{CF}_{3} \mathrm{C}_{6} \mathrm{~F}_{11}: \mathrm{THF}$ & $\begin{array}{l}84.0: 16.0 \\
(P=5.26)\end{array}$ & gravimet & 13 \\
\hline VI-20c & & $\mathrm{C}_{6} \mathrm{~F}_{14}: \mathrm{CH}_{3} \mathrm{COCH}_{3}$ & $\begin{array}{l}76.9: 23.1 \\
(P=3.33)\end{array}$ & gravimet & 13 \\
\hline VI-21c & & $\mathrm{CF}_{3} \mathrm{C}_{6} \mathrm{~F}_{11}: \mathrm{CH}_{3} \mathrm{COCH}_{3}$ & $\begin{array}{l}86.3: 13.7 \\
(P=6.28)\end{array}$ & gravimet & 13 \\
\hline VI-22c & & $\mathrm{C}_{6} \mathrm{~F}_{14}: \mathrm{CH}_{3} \mathrm{CN}$ & $\begin{array}{l}86.5: 13.5 \\
(P=6.42)\end{array}$ & gravimet & 13 \\
\hline VI-23c & & $\mathrm{CF}_{3} \mathrm{C}_{6} \mathrm{~F}_{11}: \mathrm{CH}_{3} \mathrm{CN}$ & $\begin{array}{l}94.4: 5.6 \\
(P=16.9)\end{array}$ & gravimet & 13 \\
\hline VI-24c & & $\mathrm{C}_{6} \mathrm{~F}_{14}: \mathrm{C}_{2} \mathrm{H}_{5} \mathrm{OH}$ & $\begin{array}{l}80.7: 19.3 \\
(P=4.19)\end{array}$ & gravimet & 13 \\
\hline VI-25c & & $\mathrm{CF}_{3} \mathrm{C}_{6} \mathrm{~F}_{11}: \mathrm{C}_{2} \mathrm{H}_{5} \mathrm{OH}$ & $\begin{array}{l}94.1: 5.9 \\
(P=16.0)\end{array}$ & gravimet & 13 \\
\hline VI-26c & & $\mathrm{C}_{6} \mathrm{~F}_{14}: \mathrm{CH}_{3} \mathrm{OH}$ & $\begin{array}{l}82.7: 17.3 \\
(P=4.78)\end{array}$ & gravimet & 13 \\
\hline VI-27c & & $\mathrm{CF}_{3} \mathrm{C}_{6} \mathrm{~F}_{11}: \mathrm{CH}_{3} \mathrm{OH}$ & $\begin{array}{l}91.5: 8.5 \\
(P=10.8)\end{array}$ & gravimet & 13 \\
\hline $\mathrm{VI}-28^{\mathrm{c}}$ & 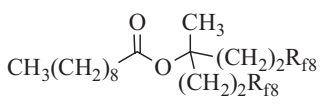 & $\mathrm{C}_{6} \mathrm{~F}_{14}: \mathrm{c}-\mathrm{C}_{6} \mathrm{H}_{12}$ & $\begin{array}{l}87.0: 13.0 \\
(P=6.72)\end{array}$ & gravimet & 13 \\
\hline VI-29c & & $\mathrm{CF}_{3} \mathrm{C}_{6} \mathrm{~F}_{11}: c-\mathrm{C}_{6} \mathrm{H}_{12}$ & $\begin{array}{l}92.5: 7.5 \\
(P=12.4)\end{array}$ & gravimet & 13 \\
\hline
\end{tabular}


6.2 Literature Data $\mid 63$

Tab. 6-1. (continued)

\begin{tabular}{|c|c|c|c|c|c|}
\hline Entry & Solute ${ }^{\mathrm{b}}$ & Solvent system & $\begin{array}{l}\text { Partitioning } \\
\text { fluorous: } \\
\text { organic (P) }\end{array}$ & Method & Ref. \\
\hline VI-30 & & $\mathrm{C}_{6} \mathrm{~F}_{14}: \mathrm{CH}_{3} \mathrm{C}_{6} \mathrm{H}_{5}$ & $\begin{array}{l}84.1: 15.9 \\
(P=5.28)\end{array}$ & gravimet & 13 \\
\hline VI-31 ${ }^{c}$ & & $\mathrm{CF}_{3} \mathrm{C}_{6} \mathrm{~F}_{11}: \mathrm{CH}_{3} \mathrm{C}_{6} \mathrm{H}_{5}$ & $\begin{array}{l}94.5: 5.5 \\
(P=17.2)\end{array}$ & gravimet & 13 \\
\hline VI-32c & & $\mathrm{C}_{6} \mathrm{~F}_{14}: \mathrm{CH}_{2} \mathrm{Cl}_{2}$ & $\begin{array}{l}81.9: 18.1 \\
(P=4.53)\end{array}$ & gravimet & 13 \\
\hline VI-33c & & $\mathrm{CF}_{3} \mathrm{C}_{6} \mathrm{~F}_{11}: \mathrm{CH}_{2} \mathrm{Cl}_{2}$ & $\begin{array}{l}93.2: 6.8 \\
(P=13.6)\end{array}$ & gravimet & 13 \\
\hline VI-34 ${ }^{c}$ & & $\mathrm{C}_{6} \mathrm{~F}_{14}: \mathrm{CHCl}_{3}$ & $\begin{array}{l}72.5: 27.5 \\
(P=2.63)\end{array}$ & gravimet & 13 \\
\hline VI-35c & & $\mathrm{CF}_{3} \mathrm{C}_{6} \mathrm{~F}_{11}: \mathrm{CHCl}_{3}$ & $\begin{array}{l}85.2: 14.8 \\
(P=5.74)\end{array}$ & gravimet & 13 \\
\hline VI-36c & & $\mathrm{C}_{6} \mathrm{~F}_{14}: \mathrm{CH}_{3} \mathrm{CO}_{2} \mathrm{C}_{2} \mathrm{H}_{5}$ & $\begin{array}{l}73.3: 26.7 \\
(P=2.74)\end{array}$ & gravimet & 13 \\
\hline VI-37c & & $\mathrm{CF}_{3} \mathrm{C}_{6} \mathrm{~F}_{11}: \mathrm{CH}_{3} \mathrm{CO}_{2} \mathrm{C}_{2} \mathrm{H}_{5}$ & $\begin{array}{l}90.8: 9.2 \\
(P=9.86)\end{array}$ & gravimet & 13 \\
\hline VI-38c & & $\mathrm{C}_{6} \mathrm{~F}_{14}: \mathrm{THF}$ & $\begin{array}{l}\text { 63.0:37.0 } \\
(P=1.70)\end{array}$ & gravimet & 13 \\
\hline VI-39c & & $\mathrm{CF}_{3} \mathrm{C}_{6} \mathrm{~F}_{11}: \mathrm{THF}$ & $\begin{array}{l}82.7: 17.3 \\
(P=4.79)\end{array}$ & gravimet & 13 \\
\hline VI-40 & & $\mathrm{C}_{6} \mathrm{~F}_{14}: \mathrm{CH}_{3} \mathrm{COCH}_{3}$ & $\begin{array}{l}74.5: 25.5 \\
(P=2.92)\end{array}$ & gravimet & 13 \\
\hline VI-41 ${ }^{c}$ & & $\mathrm{CF}_{3} \mathrm{C}_{6} \mathrm{~F}_{11}: \mathrm{CH}_{3} \mathrm{COCH}_{3}$ & $\begin{array}{l}84.7: 15.3 \\
(P=5.55)\end{array}$ & gravimet & 13 \\
\hline VI-42c & & $\mathrm{C}_{6} \mathrm{~F}_{14}: \mathrm{CH}_{3} \mathrm{CN}$ & $\begin{array}{l}91.8: 8.2 \\
(P=11.2)\end{array}$ & gravimet & 13 \\
\hline VI-43c & & $\mathrm{CF}_{3} \mathrm{C}_{6} \mathrm{~F}_{11}: \mathrm{CH}_{3} \mathrm{CN}$ & $\begin{array}{l}96.8: 3.2 \\
(P=29.9)\end{array}$ & gravimet & 13 \\
\hline VI-44c & & $\mathrm{C}_{6} \mathrm{~F}_{14}: \mathrm{C}_{2} \mathrm{H}_{5} \mathrm{OH}$ & $\begin{array}{l}85.7: 14.3 \\
(P=5.98)\end{array}$ & gravimet & 13 \\
\hline VI-45 & & $\mathrm{CF}_{3} \mathrm{C}_{6} \mathrm{~F}_{11}: \mathrm{C}_{2} \mathrm{H}_{5} \mathrm{OH}$ & $\begin{array}{l}91.7: 8.3 \\
(P=11.0)\end{array}$ & gravimet & 13 \\
\hline VI-46 & & $\mathrm{C}_{6} \mathrm{~F}_{14}: \mathrm{CH}_{3} \mathrm{OH}$ & $\begin{array}{l}89.9: 10.1 \\
(P=8.89)\end{array}$ & gravimet & 13 \\
\hline VI-47c & & $\mathrm{CF}_{3} \mathrm{C}_{6} \mathrm{~F}_{11}: \mathrm{CH}_{3} \mathrm{OH}$ & $\begin{array}{l}96.5: 3.5 \\
(P=27.3)\end{array}$ & gravimet & 13 \\
\hline VI-48c & 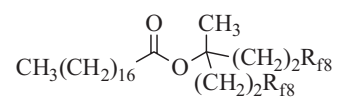 & $\mathrm{C}_{6} \mathrm{~F}_{14}: \mathrm{c}-\mathrm{C}_{6} \mathrm{H}_{12}$ & $\begin{array}{l}48: 52 \\
(P=0.91)\end{array}$ & gravimet & 13 \\
\hline VI-49c & & $\mathrm{CF}_{3} \mathrm{C}_{6} \mathrm{~F}_{11}: c-\mathrm{C}_{6} \mathrm{H}_{12}$ & $\begin{array}{l}74.1: 25.9 \\
(P=2.86)\end{array}$ & gravimet & 13 \\
\hline VI-50 & & $\mathrm{C}_{6} \mathrm{~F}_{14}: \mathrm{CH}_{3} \mathrm{C}_{6} \mathrm{H}_{5}$ & $\begin{array}{l}53.1: 46.9 \\
(P=1.13)\end{array}$ & gravimet & 13 \\
\hline VI-51 ${ }^{c}$ & & $\mathrm{CF}_{3} \mathrm{C}_{6} \mathrm{~F}_{11}: \mathrm{CH}_{3} \mathrm{C}_{6} \mathrm{H}_{5}$ & $\begin{array}{l}79.3: 20.7 \\
(\boldsymbol{P}=3.84)\end{array}$ & gravimet & 13 \\
\hline
\end{tabular}


646 Partition Coefficients Involving Fluorous Solvents

Tab. 6-1. (continued)

\begin{tabular}{|c|c|c|c|c|c|}
\hline Entry & Solute $^{\mathrm{b}}$ & Solvent system & $\begin{array}{l}\text { Partitioning } \\
\text { fluorous: } \\
\text { organic (P) }\end{array}$ & Method & Ref. \\
\hline VI-52c & & $\mathrm{C}_{6} \mathrm{~F}_{14}: \mathrm{CH}_{2} \mathrm{Cl}_{2}$ & $\begin{array}{l}50.0: 45.0 \\
(P=1.22)\end{array}$ & gravimet & 13 \\
\hline VI-53c & & $\mathrm{CF}_{3} \mathrm{C}_{6} \mathrm{~F}_{11}: \mathrm{CH}_{2} \mathrm{Cl}_{2}$ & $\begin{array}{l}82.4: 17.6 \\
(P=4.68)\end{array}$ & gravimet & 13 \\
\hline VI-54 & & $\mathrm{C}_{6} \mathrm{~F}_{14}: \mathrm{CHCl}_{3}$ & $\begin{array}{l}35: 65 \\
(P=0.53)\end{array}$ & gravimet & 13 \\
\hline VI-55 & & $\mathrm{CF}_{3} \mathrm{C}_{6} \mathrm{~F}_{11}: \mathrm{CHCl}_{3}$ & $\begin{array}{l}\text { 62.1:37.9 } \\
(P=1.64)\end{array}$ & gravimet & 13 \\
\hline VI-56 & & $\mathrm{C}_{6} \mathrm{~F}_{14}: \mathrm{CH}_{3} \mathrm{CO}_{2} \mathrm{C}_{2} \mathrm{H}_{5}$ & $\begin{array}{l}51.5: 48.5 \\
(P=1.06)\end{array}$ & gravimet & 13 \\
\hline VI-57c & & $\mathrm{CF}_{3} \mathrm{C}_{6} \mathrm{~F}_{11}: \mathrm{CH}_{3} \mathrm{CO}_{2} \mathrm{C}_{2} \mathrm{H}_{5}$ & $\begin{array}{l}67.5: 32.5 \\
(P=2.08)\end{array}$ & gravimet & 13 \\
\hline VI-58 & & $\mathrm{C}_{6} \mathrm{~F}_{14}: \mathrm{THF}$ & $\begin{array}{l}33: 67 \\
(P=0.50)\end{array}$ & gravimet & 13 \\
\hline VI-59c & & $\mathrm{CF}_{3} \mathrm{C}_{6} \mathrm{~F}_{11}: \mathrm{THF}$ & $\begin{array}{l}51.0: 49.0 \\
(P=1.04)\end{array}$ & gravimet & 13 \\
\hline VI-60c & & $\mathrm{C}_{6} \mathrm{~F}_{14}: \mathrm{CH}_{3} \mathrm{COCH}_{3}$ & $\begin{array}{l}\text { 63.9:36.1 } \\
(P=1.77)\end{array}$ & gravimet & 13 \\
\hline VI-61 ${ }^{c}$ & & $\mathrm{CF}_{3} \mathrm{C}_{6} \mathrm{~F}_{11}: \mathrm{CH}_{3} \mathrm{COCH}_{3}$ & $\begin{array}{l}72.9: 27.1 \\
(P=2.69)\end{array}$ & gravimet & 13 \\
\hline VI-62 & & $\mathrm{C}_{6} \mathrm{~F}_{14}: \mathrm{CH}_{3} \mathrm{CN}$ & $\begin{array}{l}93.5: 6.5 \\
(P=14.3)\end{array}$ & gravimet & 13 \\
\hline VI-63c & & $\mathrm{CF}_{3} \mathrm{C}_{6} \mathrm{~F}_{11}: \mathrm{CH}_{3} \mathrm{CN}$ & $\begin{array}{l}94.3: 5.7 \\
(P=16.4)\end{array}$ & gravimet & 13 \\
\hline VI-64c & & $\mathrm{C}_{6} \mathrm{~F}_{14}: \mathrm{C}_{2} \mathrm{H}_{5} \mathrm{OH}$ & $\begin{array}{l}89.3: 10.7 \\
(P=8.35)\end{array}$ & gravimet & 13 \\
\hline VI-65c & & $\mathrm{CF}_{3} \mathrm{C}_{6} \mathrm{~F}_{11}: \mathrm{C}_{2} \mathrm{H}_{5} \mathrm{OH}$ & $\begin{array}{l}91.8: 8.2 \\
(P=11.2)\end{array}$ & gravimet & 13 \\
\hline VI-66 & & $\mathrm{C}_{6} \mathrm{~F}_{14}: \mathrm{CH}_{3} \mathrm{OH}$ & $\begin{array}{l}95.1: 4.9 \\
(P=19.5)\end{array}$ & gravimet & 13 \\
\hline VI-67c & & $\mathrm{CF}_{3} \mathrm{C}_{6} \mathrm{~F}_{11}: \mathrm{CH}_{3} \mathrm{OH}$ & $\begin{array}{l}97.1: 2.9 \\
(P=33.7)\end{array}$ & gravimet & 13 \\
\hline VI-68c & & $\mathrm{C}_{6} \mathrm{~F}_{14}: \mathrm{c}-\mathrm{C}_{6} \mathrm{H}_{12}$ & $\begin{array}{l}89.2: 10.8 \\
(P=8.26)\end{array}$ & gravimet & 13 \\
\hline VI-69c & & $\mathrm{CF}_{3} \mathrm{C}_{6} \mathrm{~F}_{11}: c-\mathrm{C}_{6} \mathrm{H}_{12}$ & $\begin{array}{l}84.3: 15.7 \\
(P=5.37)\end{array}$ & gravimet & 13 \\
\hline VI-70c & & $\mathrm{C}_{6} \mathrm{~F}_{14}: \mathrm{CH}_{3} \mathrm{C}_{6} \mathrm{H}_{5}$ & $\begin{array}{l}64.0: 36.0 \\
(P=1.78)\end{array}$ & gravimet & 13 \\
\hline VI-71 ${ }^{c}$ & & $\mathrm{CF}_{3} \mathrm{C}_{6} \mathrm{~F}_{11}: \mathrm{CH}_{3} \mathrm{C}_{6} \mathrm{H}_{5}$ & $\begin{array}{l}74.2: 25.8 \\
(\boldsymbol{P}=2.87)\end{array}$ & gravimet & 13 \\
\hline VI-72 & & $\mathrm{C}_{6} \mathrm{~F}_{14}: \mathrm{CH}_{2} \mathrm{Cl}_{2}$ & $\begin{array}{l}38: 62 \\
(P=0.61)\end{array}$ & gravimet & 13 \\
\hline VI-73c & & $\mathrm{CF}_{3} \mathrm{C}_{6} \mathrm{~F}_{11}: \mathrm{CH}_{2} \mathrm{Cl}_{2}$ & $\begin{array}{l}\text { 66.2:33.8 } \\
(P=1.96)\end{array}$ & gravimet & 13 \\
\hline
\end{tabular}


6.2 Literature Data $\mid 65$

Tab. 6-1. (continued)

\begin{tabular}{|c|c|c|c|c|c|}
\hline Entry & Solute ${ }^{\mathrm{b}}$ & Solvent system & $\begin{array}{l}\text { Partitioning } \\
\text { fluorous: } \\
\text { organic (P) }\end{array}$ & Method & Ref. \\
\hline VI-74c & & $\mathrm{C}_{6} \mathrm{~F}_{14}: \mathrm{CHCl}_{3}$ & $\begin{array}{l}41: 59 \\
(P=0.69)\end{array}$ & gravimet & 13 \\
\hline VI-75c & & $\mathrm{CF}_{3} \mathrm{C}_{6} \mathrm{~F}_{11}: \mathrm{CHCl}_{3}$ & $\begin{array}{l}46: 54 \\
(P=0.86)\end{array}$ & gravimet & 13 \\
\hline VI-76c & & $\mathrm{C}_{6} \mathrm{~F}_{14}: \mathrm{CH}_{3} \mathrm{CO}_{2} \mathrm{C}_{2} \mathrm{H}_{5}$ & $\begin{array}{l}22: 78 \\
(P=0.29)\end{array}$ & gravimet & 13 \\
\hline VI-77c & & $\mathrm{CF}_{3} \mathrm{C}_{6} \mathrm{~F}_{11}: \mathrm{CH}_{3} \mathrm{CO}_{2} \mathrm{C}_{2} \mathrm{H}_{5}$ & $\begin{array}{l}37: 63 \\
(P=0.58)\end{array}$ & gravimet & 13 \\
\hline VI-78c & & $\mathrm{C}_{6} \mathrm{~F}_{14}: \mathrm{THF}$ & $\begin{array}{l}27: 73 \\
(P=0.37)\end{array}$ & gravimet & 13 \\
\hline VI-79c & & $\mathrm{CF}_{3} \mathrm{C}_{6} \mathrm{~F}_{11}: \mathrm{THF}$ & $\begin{array}{l}35: 65 \\
(P=0.55)\end{array}$ & gravimet & 13 \\
\hline VI-80c & & $\mathrm{C}_{6} \mathrm{~F}_{14}: \mathrm{CH}_{3} \mathrm{COCH}_{3}$ & $\begin{array}{l}20: 80 \\
(P=0.25)\end{array}$ & gravimet & 13 \\
\hline VI-81 ${ }^{c}$ & & $\mathrm{CF}_{3} \mathrm{C}_{6} \mathrm{~F}_{11}: \mathrm{CH}_{3} \mathrm{COCH}_{3}$ & $\begin{array}{l}36: 64 \\
(P=0.57)\end{array}$ & gravimet & 13 \\
\hline VI-82c & & $\mathrm{C}_{6} \mathrm{~F}_{14}: \mathrm{CH}_{3} \mathrm{CN}$ & $\begin{array}{l}77.4: 22.6 \\
(P=3.42)\end{array}$ & gravimet & 13 \\
\hline VI-83c & & $\mathrm{CF}_{3} \mathrm{C}_{6} \mathrm{~F}_{11}: \mathrm{CH}_{3} \mathrm{CN}$ & $\begin{array}{l}82.8: 17.2 \\
(P=4.81)\end{array}$ & gravimet & 13 \\
\hline VI-84 & & $\mathrm{C}_{6} \mathrm{~F}_{14}: \mathrm{C}_{2} \mathrm{H}_{5} \mathrm{OH}$ & $\begin{array}{l}76.7: 23.3 \\
(P=3.29)\end{array}$ & gravimet & 13 \\
\hline VI-85c & & $\mathrm{CF}_{3} \mathrm{C}_{6} \mathrm{~F}_{11}: \mathrm{C}_{2} \mathrm{H}_{5} \mathrm{OH}$ & $\begin{array}{l}81.7: 18.3 \\
(P=4.46)\end{array}$ & gravimet & 13 \\
\hline VI-86c & & $\mathrm{C}_{6} \mathrm{~F}_{14}: \mathrm{CH}_{3} \mathrm{OH}$ & $\begin{array}{l}82.3: 17.7 \\
(P=4.65)\end{array}$ & gravimet & 13 \\
\hline VI-87c & & $\mathrm{CF}_{3} \mathrm{C}_{6} \mathrm{~F}_{11}: \mathrm{CH}_{3} \mathrm{OH}$ & $\begin{array}{l}91.7: 8.3 \\
(P=11.1)\end{array}$ & gravimet & 13 \\
\hline VI-88c & & $\mathrm{C}_{6} \mathrm{~F}_{14}: \mathrm{C}-\mathrm{C}_{6} \mathrm{H}_{12}$ & $\begin{array}{l}93.6: 6.4 \\
(P=14.62)\end{array}$ & gravimet & 13 \\
\hline VI-89c & & $\mathrm{CF}_{3} \mathrm{C}_{6} \mathrm{~F}_{11}: c-\mathrm{C}_{6} \mathrm{H}_{12}$ & $\begin{array}{l}93.3: 6.7 \\
(P=13.92)\end{array}$ & gravimet & 13 \\
\hline VI-90c & & $\mathrm{C}_{6} \mathrm{~F}_{14}: \mathrm{CH}_{3} \mathrm{C}_{6} \mathrm{H}_{5}$ & $\begin{array}{l}84.6: 15.4 \\
(P=5.49)\end{array}$ & gravimet & 13 \\
\hline VI-91c & & $\mathrm{CF}_{3} \mathrm{C}_{6} \mathrm{~F}_{11}: \mathrm{CH}_{3} \mathrm{C}_{6} \mathrm{H}_{5}$ & $\begin{array}{l}90.5: 9.5 \\
(P=9.53)\end{array}$ & gravimet & 13 \\
\hline VI-92c & & $\mathrm{C}_{6} \mathrm{~F}_{14}: \mathrm{CH}_{2} \mathrm{Cl}_{2}$ & $\begin{array}{l}80.0: 20.0 \\
(P=4.00)\end{array}$ & gravimet & 13 \\
\hline VI-93c & & $\mathrm{CF}_{3} \mathrm{C}_{6} \mathrm{~F}_{11}: \mathrm{CH}_{2} \mathrm{Cl}_{2}$ & $\begin{array}{l}86.1: 13.9 \\
(P=6.21)\end{array}$ & gravimet & 13 \\
\hline VI-94c & & $\mathrm{C}_{6} \mathrm{~F}_{14}: \mathrm{CHCl}_{3}$ & $\begin{array}{l}71.8: 28.2 \\
(P=2.55)\end{array}$ & gravimet & 13 \\
\hline VI-95c & & $\mathrm{CF}_{3} \mathrm{C}_{6} \mathrm{~F}_{11}: \mathrm{CHCl}_{3}$ & $\begin{array}{l}73.0: 27.0 \\
(P=2.70)\end{array}$ & gravimet & 13 \\
\hline
\end{tabular}


$66 \mid 6$ Partition Coefficients Involving Fluorous Solvents

Tab. 6-1. (continued)

\begin{tabular}{|c|c|c|c|c|c|}
\hline Entry & Solute ${ }^{\mathrm{b}}$ & Solvent system & $\begin{array}{l}\text { Partitioning } \\
\text { fluorous: } \\
\text { organic (P) }\end{array}$ & Method & Ref. \\
\hline VI-96c & & $\mathrm{C}_{6} \mathrm{~F}_{14}: \mathrm{CH}_{3} \mathrm{CO}_{2} \mathrm{C}_{2} \mathrm{H}_{5}$ & $\begin{array}{l}56.9: 43.1 \\
(P=1.32)\end{array}$ & gravimet & 13 \\
\hline VI-97c & & $\mathrm{CF}_{3} \mathrm{C}_{6} \mathrm{~F}_{11}: \mathrm{CH}_{3} \mathrm{CO}_{2} \mathrm{C}_{2} \mathrm{H}_{5}$ & $\begin{array}{l}\text { 67.1:32.9 } \\
(P=2.04)\end{array}$ & gravimet & 13 \\
\hline VI-98c & & $\mathrm{C}_{6} \mathrm{~F}_{14}: \mathrm{THF}$ & $\begin{array}{l}56.5: 43.5 \\
(P=1.30)\end{array}$ & gravimet & 13 \\
\hline VI-99c & & $\mathrm{CF}_{3} \mathrm{C}_{6} \mathrm{~F}_{11}: \mathrm{THF}$ & $\begin{array}{l}75.1: 24.9 \\
(P=3.02)\end{array}$ & gravimet & 13 \\
\hline VI-100 & & $\mathrm{C}_{6} \mathrm{~F}_{14}: \mathrm{CH}_{3} \mathrm{COCH}_{3}$ & $\begin{array}{l}60.0: 40.0 \\
(P=1.50)\end{array}$ & gravimet & 13 \\
\hline VI-101 ${ }^{c}$ & & $\mathrm{CF}_{3} \mathrm{C}_{6} \mathrm{~F}_{11}: \mathrm{CH}_{3} \mathrm{COCH}_{3}$ & $\begin{array}{l}71.1: 28.9 \\
(P=2.46)\end{array}$ & gravimet & 13 \\
\hline VI-102 & & $\mathrm{C}_{6} \mathrm{~F}_{14}: \mathrm{CH}_{3} \mathrm{CN}$ & $\begin{array}{l}90.0: 10.0 \\
(\boldsymbol{P}=9.00)\end{array}$ & gravimet & 13 \\
\hline VI-103c & & $\mathrm{CF}_{3} \mathrm{C}_{6} \mathrm{~F}_{11}: \mathrm{CH}_{3} \mathrm{CN}$ & $\begin{array}{l}94.2: 5.8 \\
(P=16.2)\end{array}$ & gravimet & 13 \\
\hline VI-104c & & $\mathrm{C}_{6} \mathrm{~F}_{14}: \mathrm{C}_{2} \mathrm{H}_{5} \mathrm{OH}$ & $\begin{array}{l}88.5: 11.5 \\
(P=7.69)\end{array}$ & gravimet & 13 \\
\hline VI-105c & & $\mathrm{CF}_{3} \mathrm{C}_{6} \mathrm{~F}_{11}: \mathrm{C}_{2} \mathrm{H}_{5} \mathrm{OH}$ & $\begin{array}{l}91.1: 8.9 \\
(P=10.24)\end{array}$ & gravimet & 13 \\
\hline VI-106 & & $\mathrm{C}_{6} \mathrm{~F}_{14}: \mathrm{CH}_{3} \mathrm{OH}$ & $\begin{array}{l}92.1: 7.9 \\
(P=11.7)\end{array}$ & gravimet & 13 \\
\hline VI-107c & & $\mathrm{CF}_{3} \mathrm{C}_{6} \mathrm{~F}_{11}: \mathrm{CH}_{3} \mathrm{OH}$ & $\begin{array}{l}95.4: 4.6 \\
(P=20.7)\end{array}$ & gravimet & 13 \\
\hline VI-108c & $\mathrm{R}_{\mathrm{f} 7} \mathrm{C}(\mathrm{O}) \mathrm{NH}\left(\mathrm{CH}_{3}\right)$ & $\mathrm{CF}_{3} \mathrm{C}_{6} \mathrm{~F}_{11}: \mathrm{CH}_{3} \mathrm{C}_{6} \mathrm{H}_{5}$ & $\begin{array}{l}55: 45 \\
(P=1.2)^{\mathrm{d}}\end{array}$ & GLC & $4 a$ \\
\hline VI-109c & $\mathrm{R}_{\mathrm{f} 7} \mathrm{C}(\mathrm{O}) \mathrm{N}\left(\mathrm{CH}_{3}\right)_{2}$ & $\mathrm{CF}_{3} \mathrm{C}_{6} \mathrm{~F}_{11}: \mathrm{CH}_{3} \mathrm{C}_{6} \mathrm{H}_{5}$ & $\begin{array}{l}58: 42 \\
(P=1.4)^{d}\end{array}$ & GLC & $4 a$ \\
\hline VI-110 & $\mathrm{O} \smile \mathrm{N}-\mathrm{C}(\mathrm{O}) \mathrm{R}_{\mathrm{F} 7}$ & $\mathrm{CF}_{3} \mathrm{C}_{6} \mathrm{~F}_{11}: \mathrm{CH}_{3} \mathrm{C}_{6} \mathrm{H}_{5}$ & $\begin{array}{l}35: 65 \\
(P=0.54)^{d}\end{array}$ & GLC & $4 a$ \\
\hline VI-111 ${ }^{c}$ & {$\left[\mathrm{R}_{\mathrm{f} 8}\left(\mathrm{CH}_{2}\right)_{3}\right]_{2} \mathrm{C}\left(\mathrm{CO}_{2} \mathrm{CH}_{2} \mathrm{CH}_{3}\right)_{2}$} & $\mathrm{CF}_{3} \mathrm{C}_{6} \mathrm{~F}_{11}: \mathrm{CH}_{3} \mathrm{C}_{6} \mathrm{H}_{5}$ & $\begin{array}{l}85.1: 14.9 \\
(P=5.70)^{\mathrm{d}}\end{array}$ & gravimet & 14 \\
\hline VI-112 & $\begin{array}{l}{\left[\mathrm{R}_{\mathrm{f} 8}\left(\mathrm{CH}_{2}\right)_{3}\right]_{2} \mathrm{CH}\left(\mathrm{CO}_{2} \mathrm{H}\right)} \\
{\left[\left(\mathrm{R}_{\mathrm{f} 6}\left(\mathrm{CH}_{2}\right)_{2}\right)_{3} \mathrm{Si}\left(\mathrm{CH}_{2}\right)_{3}\right] \mathrm{NRR}^{\prime}} \\
\mathrm{O}\end{array}$ & $\mathrm{CF}_{3} \mathrm{C}_{6} \mathrm{~F}_{11}: \mathrm{CH}_{3} \mathrm{C}_{6} \mathrm{H}_{5}$ & $\begin{array}{l}97.1: 2.9 \\
(P=33.1)^{\mathrm{d}}\end{array}$ & gravimet & 14 \\
\hline VI-113i & $\mathrm{H}, \mathrm{R}^{\prime}$ & $\mathrm{C}_{6} \mathrm{~F}_{14}: \mathrm{CHCl}_{3}$ & $\begin{array}{l}21: 79 \\
(P=0.27)\end{array}$ & $\begin{array}{l}\text { gravimet or } \\
\text { HPLC }\end{array}$ & 15 \\
\hline VI-114j & & $\mathrm{C}_{6} \mathrm{~F}_{14}: \mathrm{CH}_{3} \mathrm{CN}$ & $\begin{array}{l}50.0: 50.0 \\
(P=1.00)\end{array}$ & $\begin{array}{l}\text { gravimet or } \\
\text { HPLC }\end{array}$ & 15 \\
\hline VI-115i & & $\mathrm{C}_{6} \mathrm{~F}_{14}: \mathrm{CH}_{3} \mathrm{OH}$ & $\begin{array}{l}21: 79 \\
(P=0.27)\end{array}$ & $\begin{array}{l}\text { gravimet or } \\
\text { HPLC }\end{array}$ & 15 \\
\hline
\end{tabular}


6.2 Literature Data 67

Tab. 6-1. (continued)

Entry Solute

Solvent system

Partitioning Method Ref.

fluorous:

organic $(P)$

VI-116 $\mathrm{R}=\mathrm{H}, \mathrm{R}^{\prime}=\overbrace{\mathrm{CH}_{3}}$

VI-117i

VI-118 $\mathrm{R}=\mathrm{CH}_{3}, \mathrm{R}^{\prime}=$

VI-119i

$\left[\left(\mathrm{R}_{\mathrm{f} 6}\left(\mathrm{CH}_{2}\right)_{2}\right)_{3} \mathrm{Si}\left(\mathrm{CH}_{2}\right)_{3}\right]_{2} \mathrm{NR}$

VI-120<smiles>[R]C(=O)c1ccc(C)cc1</smiles>

VI-121

VI-122

VI-123

VI-124i

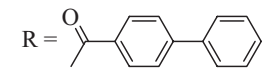

VI-125

VI-126

VI-127i<smiles>[R]C(=O)Nc1ccc(C)cc1</smiles>

VI-128

VI-129

VI-130<smiles>[R]C(=O)NS(=O)c1ccc(C)cc1</smiles>

VI-131
$\mathrm{C}_{6} \mathrm{~F}_{14}: \mathrm{CH}_{3} \mathrm{CN}$

$\mathrm{C}_{6} \mathrm{~F}_{14}: \mathrm{CH}_{3} \mathrm{OH}$

$\mathrm{C}_{6} \mathrm{~F}_{14}: \mathrm{CH}_{3} \mathrm{CN}$

$\mathrm{C}_{6} \mathrm{~F}_{14}: \mathrm{CH}_{3} \mathrm{OH}$

$\mathrm{C}_{6} \mathrm{~F}_{14}: \mathrm{CHCl}_{3}$

$\mathrm{C}_{6} \mathrm{~F}_{14}: \mathrm{CH}_{3} \mathrm{CN}$

$\mathrm{C}_{6} \mathrm{~F}_{14}: \mathrm{CH}_{3} \mathrm{OH}$

$\mathrm{C}_{6} \mathrm{~F}_{14}:$ THF

$\mathrm{C}_{6} \mathrm{~F}_{14}: \mathrm{CHCl}_{3}$

$\mathrm{C}_{6} \mathrm{~F}_{14}: \mathrm{CH}_{3} \mathrm{OH}$

$\mathrm{C}_{6} \mathrm{~F}_{14}: \mathrm{CHCl}_{3}$

$\mathrm{C}_{6} \mathrm{~F}_{14}: \mathrm{CH}_{3} \mathrm{CN}$

$\mathrm{C}_{6} \mathrm{~F}_{14}: \mathrm{CH}_{3} \mathrm{OH}$

$\mathrm{C}_{6} \mathrm{~F}_{14}:$ THF

$\mathrm{C}_{6} \mathrm{~F}_{14}: \mathrm{CHCl}_{3}$

$\mathrm{C}_{6} \mathrm{~F}_{14}: \mathrm{THF}$
55:45

$(P=1.2)$

$57: 43$

$(P=1.3)$

64:36

$(P=1.8)$

73:27

$(P=2.7)$

96:4

$(P=26)$

gravimet or 15

HPLC

$>99.0:<1.0$ gravimet or 15

$(P>100) \quad$ HPLC

$>99.0:<1.0$ gravimet or 15

$(\boldsymbol{P}>100) \quad$ HPLC

90:10 gravimet or 15

$(\boldsymbol{P}=\mathbf{9 . 0}) \quad$ HPLC

86:14 gravimet or 15

$(P=6)$

HPLC

$>99.0:<1.0$ gravimet or 15

$(P>100) \quad$ HPLC

96:4 gravimet or 15

$(P=27)$

HPLC

$>98:<2$ gravimet or 15

$(P>50) \quad$ HPLC

$>99.0:<1.0$ gravimet or 15

$(P>100) \quad$ HPLC

92:8

$(P=11)$

gravimet or 15

HPLC

97:3 gravimet or 15

$(\boldsymbol{P}=37) \quad$ HPLC

94:6 gravimet or 15

$(\boldsymbol{P}=16) \quad$ HPLC

for further compounds see X, XIV, $\mathrm{XV}$ and Table 6-2 
$68 \mid 6$ Partition Coefficients Involving Fluorous Solvents

Tab. 6-1. (continued)

\begin{tabular}{|c|c|c|c|c|c|}
\hline Entry & Solute & Solvent system & $\begin{array}{l}\text { Partitioning } \\
\text { fluorous: } \\
\text { organic (P) }\end{array}$ & Method & Ref. \\
\hline VII & Arenes, Aryl Halides, Phenols & & & & \\
\hline VII-1 ${ }^{\mathrm{c}}$ & $\mathrm{C}_{6} \mathrm{H}_{6}$ & $\mathrm{CF}_{3} \mathrm{C}_{6} \mathrm{~F}_{11}: \mathrm{CH}_{3} \mathrm{C}_{6} \mathrm{H}_{5}$ & $\begin{array}{l}\text { 6:94 } \\
(P=0.063)^{\mathrm{d}}\end{array}$ & GLC & $4 a$ \\
\hline VII-2 & $\mathrm{C}_{6} \mathrm{HF}_{5}$ & $\mathrm{CF}_{3} \mathrm{C}_{6} \mathrm{~F}_{11}: \mathrm{CH}_{3} \mathrm{C}_{6} \mathrm{H}_{5}$ & $\begin{array}{l}22.4: 77.6 \\
(P=0.289)\end{array}$ & GLC & 1 \\
\hline VII-3 & $\mathrm{C}_{6} \mathrm{~F}_{6}$ & $\mathrm{CF}_{3} \mathrm{C}_{6} \mathrm{~F}_{11}: \mathrm{CH}_{3} \mathrm{C}_{6} \mathrm{H}_{5}$ & $\begin{array}{l}28.0: 72.0 \\
(P=0.389)\end{array}$ & GLC & 1 \\
\hline VII-4 & $-\mathrm{CH}_{2} \mathrm{CH}_{3}$ & $\mathrm{CF}_{3} \mathrm{C}_{6} \mathrm{~F}_{11}: \mathrm{CH}_{3} \mathrm{C}_{6} \mathrm{H}_{5}$ & $\begin{array}{l}\text { 1.2:98.8 } \\
(P=0.012)\end{array}$ & GLC & 1 \\
\hline VII-5 & $\left(\mathrm{CH}_{2}\right)_{11} \mathrm{CH}_{3}$ & $\mathrm{CF}_{3} \mathrm{C}_{6} \mathrm{~F}_{11}: \mathrm{CH}_{3} \mathrm{C}_{6} \mathrm{H}_{5}$ & $\begin{array}{l}\mathbf{0 . 9 : 9 9 . 1} \\
(P=0.009)\end{array}$ & GLC & 1 \\
\hline VII-6c & & $\mathrm{CF}_{3} \mathrm{C}_{6} \mathrm{~F}_{11}: \mathrm{CH}_{3} \mathrm{C}_{6} \mathrm{H}_{5}$ & $\begin{array}{l}12.4: 87.6 \\
(P=0.142)^{\mathrm{d}}\end{array}$ & GLC & $4 a$ \\
\hline VII-7 ${ }^{\mathrm{i}}$ & & $\mathrm{C}_{6} \mathrm{~F}_{14}: \mathrm{CH}_{3} \mathrm{CN}$ & $\begin{array}{l}12: 88 \\
(P=0.13)\end{array}$ & GLC & 12 \\
\hline VII-8 ${ }^{\mathrm{i}}$ & & $\mathrm{C}_{6} \mathrm{~F}_{14}: \mathrm{CH}_{3} \mathrm{OH}$ & $\begin{array}{l}17: 83 \\
(P=0.21)\end{array}$ & GLC & 12 \\
\hline VII-9i & & $\mathrm{C}_{6} \mathrm{~F}_{14}: \mathrm{C}_{2} \mathrm{H}_{5} \mathrm{OH}$ & $\begin{array}{l}15: 85 \\
(P=0.18)\end{array}$ & GLC & 12 \\
\hline VII-10 ${ }^{\mathrm{i}}$ & & $\mathrm{C}_{6} \mathrm{~F}_{14}: \mathrm{C}_{6} \mathrm{H}_{6}$ & $\begin{array}{l}15: 85 \\
(P=0.18)\end{array}$ & GLC & 12 \\
\hline VII-11 ${ }^{\mathrm{i}}$ & & $\mathrm{C}_{6} \mathrm{~F}_{14}: \mathrm{CH}_{2} \mathrm{Cl}_{2}$ & $\begin{array}{l}14: 86 \\
(P=0.16)\end{array}$ & GLC & 12 \\
\hline VII-12 ${ }^{\mathrm{i}}$ & & $\mathrm{C}_{6} \mathrm{~F}_{14}: \mathrm{CH}_{3} \mathrm{COCH}_{3}$ & $\begin{array}{l}7: 93 \\
(P=0.08)\end{array}$ & GLC & 12 \\
\hline VII-13i & & $\mathrm{C}_{6} \mathrm{~F}_{14}: \mathrm{CH}_{3} \mathrm{CO}_{2} \mathrm{C}_{2} \mathrm{H}_{5}$ & $\begin{array}{l}12: 88 \\
(P=0.13)\end{array}$ & GLC & 12 \\
\hline VII-14 ${ }^{\mathrm{i}}$ & & $\mathrm{C}_{6} \mathrm{~F}_{14}: \mathrm{CHCl}_{3}$ & $\begin{array}{l}14: 86 \\
(P=0.16)\end{array}$ & GLC & 12 \\
\hline VII-15c & & $\mathrm{CF}_{3} \mathrm{C}_{6} \mathrm{~F}_{11}: \mathrm{CH}_{3} \mathrm{C}_{6} \mathrm{H}_{5}$ & $\begin{array}{l}63: 37 \\
(P=1.7)^{\mathrm{d}}\end{array}$ & GLC & $4 a$ \\
\hline VII-16c & & $\mathrm{CF}_{3} \mathrm{C}_{6} \mathrm{~F}_{11}: \mathrm{CH}_{3} \mathrm{C}_{6} \mathrm{H}_{5}$ & $\begin{array}{l}77.5: 22.5 \\
(P=3.46)^{\mathrm{d}}\end{array}$ & GLC & $4 a$ \\
\hline VII-17c & & $\mathrm{CF}_{3} \mathrm{C}_{6} \mathrm{~F}_{11}: \mathrm{CH}_{3} \mathrm{C}_{6} \mathrm{H}_{5}$ & $\begin{array}{l}85.4: 14.6 \\
(P=5.87)^{d}\end{array}$ & GLC & $4 a$ \\
\hline VII-18 & $-\left(\mathrm{CH}_{2}\right)_{3} \mathrm{R}_{\mathrm{f} 8}$ & $\mathrm{CF}_{3} \mathrm{C}_{6} \mathrm{~F}_{11}: \mathrm{CH}_{3} \mathrm{C}_{6} \mathrm{H}_{5}$ & $\begin{array}{l}49.5: 50.5 \\
(P=0.980)\end{array}$ & GLC & 1 \\
\hline VII-19 & $\mathrm{HO}-\left(\mathrm{CH}_{2}\right)_{2} \mathrm{R}_{\mathrm{f} 8}$ & $\mathrm{CF}_{3} \mathrm{C}_{6} \mathrm{~F}_{11}: \mathrm{CH}_{3} \mathrm{C}_{6} \mathrm{H}_{5}$ & $\begin{array}{l}20: 80 \\
(P=0.25)\end{array}$ & $\begin{array}{c}{ }^{1} \mathrm{H} \text { and }{ }^{19} \mathrm{~F} \\
\text { NMR }\end{array}$ & 16 \\
\hline
\end{tabular}


6.2 Literature Data $\mid 69$

Tab. 6-1. (continued)

\begin{tabular}{|c|c|c|c|c|c|}
\hline Entry & Solute & Solvent system & $\begin{array}{l}\text { Partitioning } \\
\text { fluorous: } \\
\text { organic (P) }\end{array}$ & Method & Ref. \\
\hline VII-20 & $-\left(\mathrm{CH}_{2}\right)_{3} \mathrm{R}_{\mathrm{f} 8}$ & $\mathrm{CF}_{3} \mathrm{C}_{6} \mathrm{~F}_{11}: \mathrm{CH}_{3} \mathrm{C}_{6} \mathrm{H}_{5}$ & $\begin{array}{l}\text { 12:88 } \\
(P=0.14)\end{array}$ & $\begin{array}{c}{ }^{1} \mathrm{H} \text { and }{ }^{19} \mathrm{~F} \\
\mathrm{NMR}\end{array}$ & 16 \\
\hline VII-21 & $\left.{ }_{2}\right)_{3} \mathrm{R}_{\mathrm{f8}}$ & $\mathrm{CF}_{3} \mathrm{C}_{6} \mathrm{~F}_{11}: \mathrm{CH}_{3} \mathrm{C}_{6} \mathrm{H}_{5}$ & $\begin{array}{l}\text { 14:86 } \\
(P=0.16)\end{array}$ & $\begin{array}{l}{ }^{1} \mathrm{H} \text { and }{ }^{19} \mathrm{~F} \\
\mathrm{NMR}\end{array}$ & 16 \\
\hline VII-22c & $\left.\mathrm{I}_{2}\right)_{3} \mathrm{R}_{\mathrm{f} 8}$ & $\mathrm{CF}_{3} \mathrm{C}_{6} \mathrm{~F}_{11}: \mathrm{CH}_{3} \mathrm{C}_{6} \mathrm{H}_{5}$ & $\begin{array}{l}55: 45 \\
(P=1.2)^{\mathrm{d}}\end{array}$ & GLC & 11 \\
\hline VII-23c & & $\mathrm{CF}_{3} \mathrm{C}_{6} \mathrm{~F}_{11}: \mathrm{CH}_{3} \mathrm{C}_{6} \mathrm{H}_{5}$ & $\begin{array}{l}35: 65 \\
(P=0.53)^{\mathrm{d}}\end{array}$ & GLC & $4 a$ \\
\hline VII-24c & $-\left(\mathrm{CH}_{2}\right)_{2} \mathrm{R}_{\mathrm{f} 6}$ & $\mathrm{CF}_{3} \mathrm{C}_{6} \mathrm{~F}_{11}: \mathrm{CH}_{3} \mathrm{C}_{6} \mathrm{H}_{5}$ & $\begin{array}{l}26.5: 73.5 \\
(P=0.360)^{d}\end{array}$ & GLC & $4 a$ \\
\hline VII-25c & $-\left(\mathrm{CH}_{2}\right)_{2} \mathrm{R}_{\mathrm{f8}}$ & $\mathrm{CF}_{3} \mathrm{C}_{6} \mathrm{~F}_{11}: \mathrm{CH}_{3} \mathrm{C}_{6} \mathrm{H}_{5}$ & $\begin{array}{l}41: 59 \\
(P=0.69)^{\mathrm{d}}\end{array}$ & GLC & $4 a$ \\
\hline VII-26c & & $\mathrm{CF}_{3} \mathrm{C}_{6} \mathrm{~F}_{11}: \mathrm{CH}_{3} \mathrm{C}_{6} \mathrm{H}_{5}$ & $\begin{array}{l}25.9: 74.1 \\
(P=0.350)^{d}\end{array}$ & GLC & $4 a$ \\
\hline VII-27c & & $\mathrm{CF}_{3} \mathrm{C}_{6} \mathrm{~F}_{11}: \mathrm{CH}_{3} \mathrm{C}_{6} \mathrm{H}_{5}$ & $\begin{array}{l}19.2: 80.8 \\
(P=0.237)^{d}\end{array}$ & GLC & $4 a$ \\
\hline VII-28c & $\geqslant\left(\mathrm{CH}_{2}\right)_{2} \mathrm{R}_{\mathrm{f} 6}$ & $\mathrm{CF}_{3} \mathrm{C}_{6} \mathrm{~F}_{11}: \mathrm{CH}_{3} \mathrm{C}_{6} \mathrm{H}_{5}$ & $\begin{array}{l}18.4: 81.6 \\
(P=0.225)^{d}\end{array}$ & GLC & $4 a$ \\
\hline VII-29c & & $\mathrm{CF}_{3} \mathrm{C}_{6} \mathrm{~F}_{11}: \mathrm{CH}_{3} \mathrm{C}_{6} \mathrm{H}_{5}$ & $\begin{array}{l}\text { 97.9:2.1 } \\
(P=46.6)\end{array}$ & GLC & 17 \\
\hline VII-30 & $\begin{array}{l}\mathrm{CF}_{3} \\
+\mathrm{Cl} \\
\mathrm{CF}_{3}\end{array}$ & $\mathrm{CF}_{3} \mathrm{C}_{6} \mathrm{~F}_{11}: \mathrm{CH}_{3} \mathrm{C}_{6} \mathrm{H}_{5}$ & $\begin{array}{l}11.5: 88.5 \\
(P=0.130)^{d}\end{array}$ & GLC & 11 \\
\hline VII-31 & $\begin{array}{l}\mathrm{CF}_{3} \\
-\mathrm{OCH}_{3} \\
\mathrm{CF}_{3}\end{array}$ & $\mathrm{CF}_{3} \mathrm{C}_{6} \mathrm{~F}_{11}: \mathrm{CH}_{3} \mathrm{C}_{6} \mathrm{H}_{5}$ & $\begin{array}{l}13.8: 86.2 \\
(P=0.160)^{\mathrm{d}}\end{array}$ & GLC & 11 \\
\hline VII-32c & $-\underset{\mathrm{CF}_{3}}{\mathrm{CF}_{3}}$ & $\mathrm{CF}_{3} \mathrm{C}_{6} \mathrm{~F}_{11}: \mathrm{CH}_{3} \mathrm{C}_{6} \mathrm{H}_{5}$ & $\begin{array}{l}35: 65 \\
(P=0.54)^{\mathrm{d}}\end{array}$ & GLC & 11 \\
\hline VII-33c & $\begin{array}{l}\mathrm{CF}_{3} \\
\underset{\mathrm{CF}_{3}}{-\mathrm{OCH}_{2} \mathrm{CF}_{\mathrm{f} 7}}\end{array}$ & $\mathrm{CF}_{3} \mathrm{C}_{6} \mathrm{~F}_{11}: \mathrm{CH}_{3} \mathrm{C}_{6} \mathrm{H}_{5}$ & $\begin{array}{l}87.0: 13.0 \\
(P=6.69)^{\mathrm{d}}\end{array}$ & GLC & 11 \\
\hline
\end{tabular}


$70 \mid 6$ Partition Coefficients Involving Fluorous Solvents

Tab. 6-1. (continued)

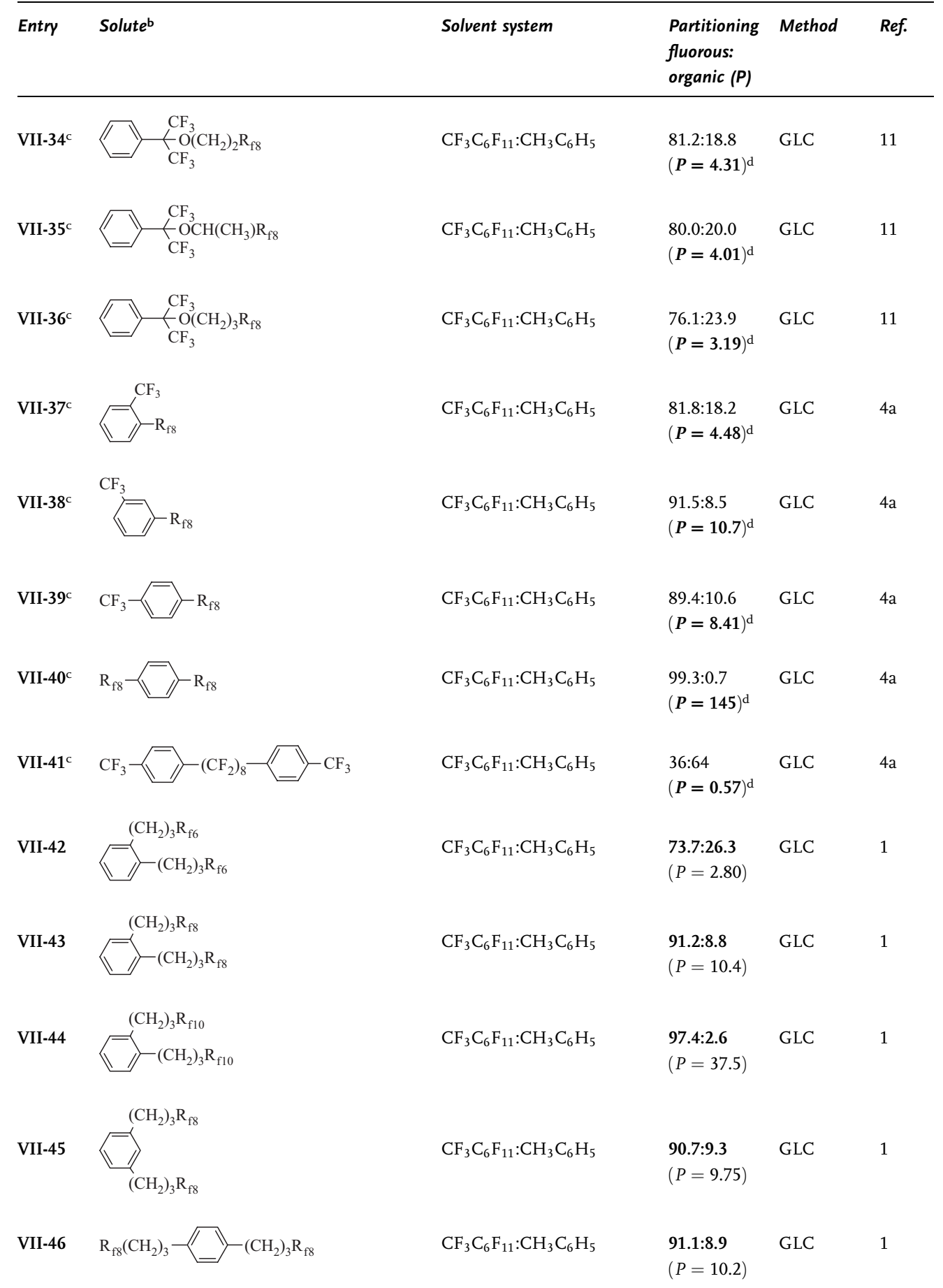


Tab. 6-1. (continued)

Entry Solute ${ }^{\mathrm{b}}$

$\mathrm{VII-47}$

VII-48

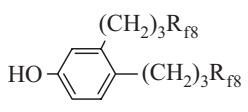

VII-49

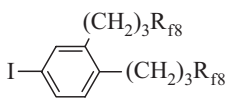

VII-50

VII-51

VII-52

VII-53

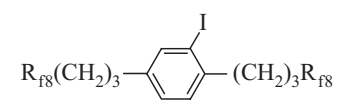

VII-54<smiles>COC(OC)(c1cccc(C(F)(F)C(F)(F)F)c1)C(F)(F)C(F)(F)F</smiles><smiles>CC(c1cccc(C(OCC(F)(F)F)(OCC(F)(F)F)C(F)(F)C(F)(F)F)c1)(C(F)(F)F)C(F)(F)F</smiles><smiles>[R10]CCOC(c1cccc(C(OCCN[R10])(C(F)(F)F)C(F)(F)F)c1)(C(F)(F)F)C(F)(F)F</smiles>

Solvent system

Partitioning Method

fluorous:

organic $(P)$

$\mathrm{CF}_{3} \mathrm{C}_{6} \mathrm{~F}_{11}: \mathrm{CH}_{3} \mathrm{C}_{6} \mathrm{H}_{5}$

70:30

$(P=2.3)$

${ }^{1} \mathrm{H}$ and ${ }^{19} \mathrm{~F} \quad 16$ NMR

$\mathrm{CF}_{3} \mathrm{C}_{6} \mathrm{~F}_{11}: \mathrm{CH}_{3} \mathrm{C}_{6} \mathrm{H}_{5}$

72:28

$(P=2.6)$

${ }^{1} \mathrm{H}$ and ${ }^{19} \mathrm{~F} \quad 16$

NMR

$\mathrm{CF}_{3} \mathrm{C}_{6} \mathrm{~F}_{11}: \mathrm{CH}_{3} \mathrm{C}_{6} \mathrm{H}_{5}$

69.5:30.5

GLC

18

$(P=2.28)$

$\mathrm{CF}_{3} \mathrm{C}_{6} \mathrm{~F}_{11}: \mathrm{CH}_{3} \mathrm{OH}$

97.0:3.0

GLC

18

$(P=32.3)$

$\mathrm{CF}_{3} \mathrm{C}_{6} \mathrm{~F}_{11}: \mathrm{CH}_{3} \mathrm{C}_{6} \mathrm{H}_{5}$

74.7:25.3

$(P=2.95)$

GLC

18

98.6:1.4

GLC

18

( $P=70.4)$

$\mathrm{CF}_{3} \mathrm{C}_{6} \mathrm{~F}_{11}: \mathrm{CH}_{3} \mathrm{C}_{6} \mathrm{H}_{5}$

73.9:26

$(P=2.83)$

GLC

18

$\mathrm{CF}_{3} \mathrm{C}_{6} \mathrm{~F}_{11}: \mathrm{CH}_{3} \mathrm{OH}$

98.0:2.0

$(P=49.0)$

GLC

18

29:71

$(\boldsymbol{P}=0.41)^{\mathrm{d}}$

GLC

11

$\mathrm{CF}_{3} \mathrm{C}_{6} \mathrm{~F}_{11}: \mathrm{CH}_{3} \mathrm{C}_{6} \mathrm{H}_{5}$

76.7:23.3 $(\boldsymbol{P}=3.29)^{\mathrm{d}}$

GLC

11

$\mathrm{CF}_{3} \mathrm{C}_{6} \mathrm{~F}_{11}: \mathrm{CH}_{3} \mathrm{C}_{6} \mathrm{H}_{5}$

99.1:0.9 $(P=107)^{\mathrm{d}}$
GLC

11
Ref.

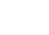

16


$72 \mid 6$ Partition Coefficients Involving Fluorous Solvents

Tab. 6-1. (continued)

Entry Solute

Solvent system

Partitioning Method Ref.

fluorous:

organic $(P)$

VII-58c

VII-59c

VII-60c

$\mathrm{R}_{\mathrm{f8}}\left(\mathrm{CH}_{2}\right)_{3}$

VII-61

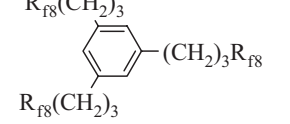

$\mathrm{R}_{\mathrm{f8}}\left(\mathrm{CH}_{2}\right)_{3}$

VII-62

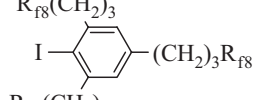

VII-63

$\mathrm{R}_{\mathrm{f} 8}\left(\mathrm{CH}_{2}\right)_{3}$

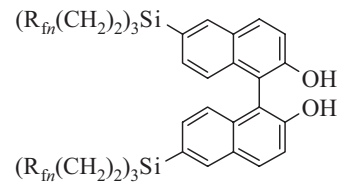

VII-649 $n=6$

VII-65g $n=6$

VII-669 $n=8$

VII-67g $n=8$

for further compounds see III, IV, VI, VIII, X, XI, XIII, XIV, XV and XVI
$\mathrm{CF}_{3} \mathrm{C}_{6} \mathrm{~F}_{11}: \mathrm{CH}_{3} \mathrm{C}_{6} \mathrm{H}_{5}$

$\begin{array}{lll}\mathrm{CF}_{3} \mathrm{C}_{6} \mathrm{~F}_{11}: \mathrm{CH}_{3} \mathrm{C}_{6} \mathrm{H}_{5} \quad \text { GLC } & \text { G:96 } \\ & (\boldsymbol{P}=\mathbf{0 . 0 4 4})^{\mathrm{d}}\end{array}$

$\mathrm{CF}_{3} \mathrm{C}_{6} \mathrm{~F}_{11}: \mathrm{CH}_{3} \mathrm{C}_{6} \mathrm{H}_{5} \quad \begin{aligned} & 98.0: 2.0 \\ & (\boldsymbol{P}=49.9)^{\mathrm{d}}\end{aligned} \quad$ GLC $\quad 11$

GLC

$4 a$

$(P=58.6)^{\mathrm{d}}$

$\mathrm{CF}_{3} \mathrm{C}_{6} \mathrm{~F}_{11}: \mathrm{CH}_{3} \mathrm{C}_{6} \mathrm{H}_{5}$

$>99.7:<0.3$ GLC

$(P>332)$

$\begin{array}{llll}\mathrm{CF}_{3} \mathrm{C}_{6} \mathrm{~F}_{11}: \mathrm{CH}_{3} \mathrm{C}_{6} \mathrm{H}_{5} & 98.0: 2.0 & \text { GLC } & 18 \\ & (P=49.0) & & \\ & & & \\ \mathrm{CF}_{3} \mathrm{C}_{6} \mathrm{~F}_{11}: \mathrm{CH}_{3} \mathrm{OH} & >99.7:<0.3 & \text { GLC } & 18 \\ & (P>332) & & \end{array}$

$\begin{array}{llll}\mathrm{C}_{6} \mathrm{~F}_{14}: \mathrm{CHCl}_{3} & 95: 5 & \text { gravimet } & 20 \\ & (P=19) & & \\ \mathrm{C}_{6} \mathrm{~F}_{14}: \mathrm{CH}_{3} \mathrm{C}_{6} \mathrm{H}_{5} & 98: 2 & \text { gravimet } & 20 \\ & (P=49) & & \\ & 99: 1 & \text { gravimet } & 20 \\ \mathrm{C}_{6} \mathrm{~F}_{14}: \mathrm{CH}_{2} \mathrm{Cl}_{2} & (P=99) & & \\ & 99: 1 & \text { gravimet } & 20 \\ \mathrm{C}_{6} \mathrm{~F}_{14}: \mathrm{CH}_{3} \mathrm{C}_{6} \mathrm{H}_{5} & (P=99) & & \\ & & & \end{array}$


6.2 Literature Data $\mid 73$

Tab. 6-1. (continued)

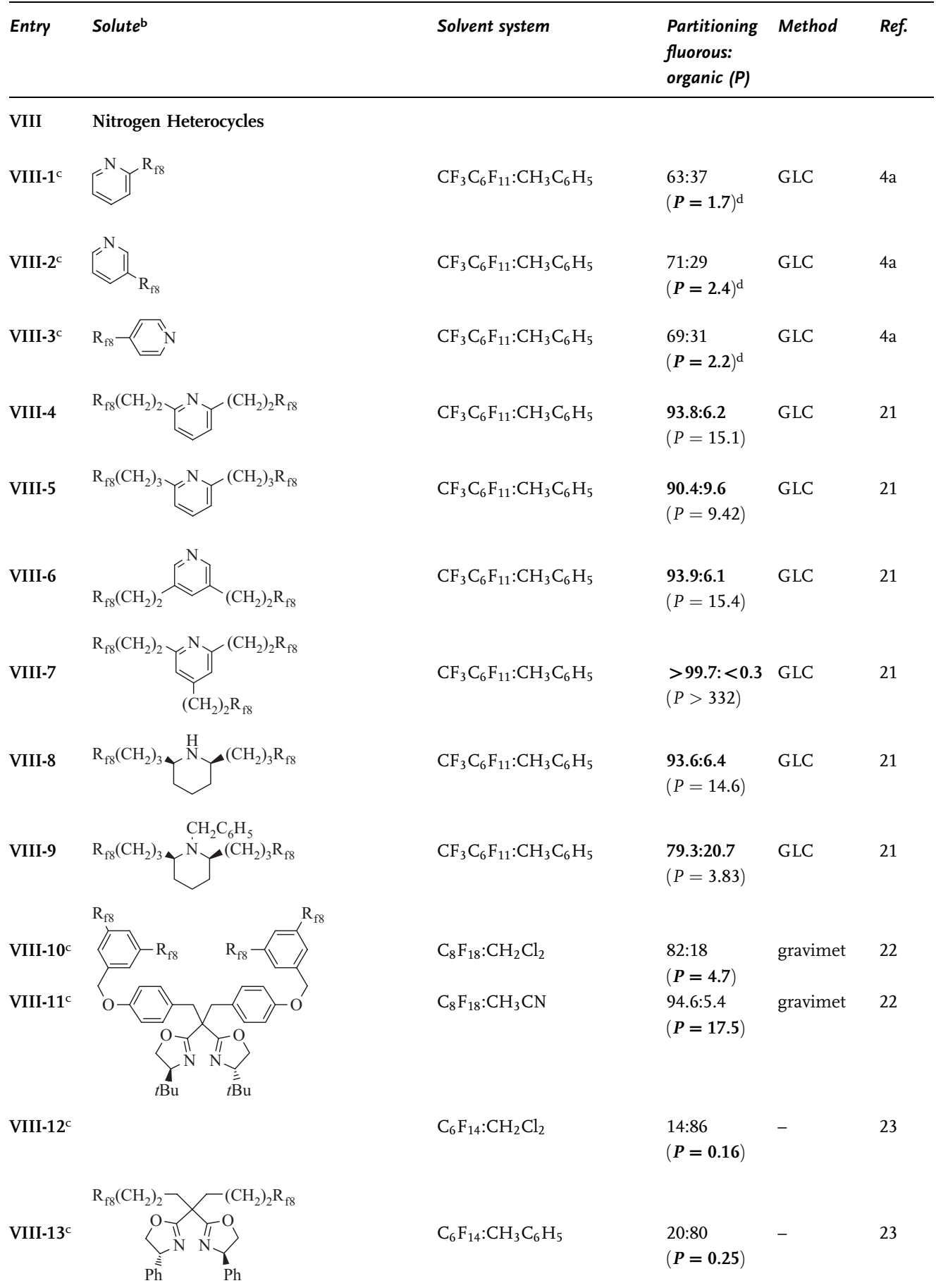




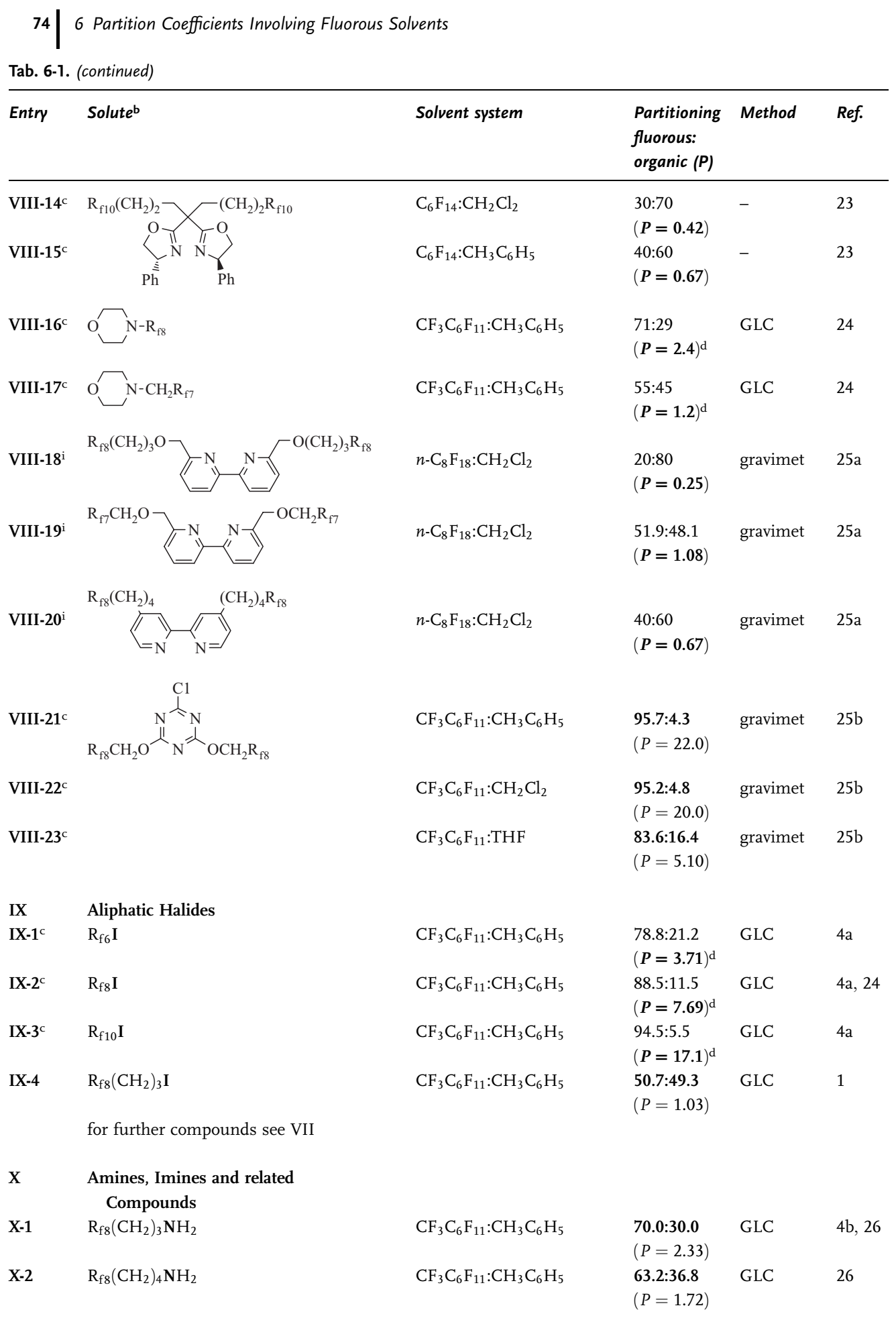


6.2 Literature Data $\mid 75$

Tab. 6-1. (continued)

\begin{tabular}{|c|c|c|c|c|c|}
\hline Entry & Solute $\mathrm{b}^{\mathrm{b}}$ & Solvent system & $\begin{array}{l}\text { Partitioning } \\
\text { fluorous: } \\
\text { organic (P) }\end{array}$ & Method & Ref. \\
\hline$X-3$ & $\mathrm{R}_{\mathrm{f} 8}\left(\mathrm{CH}_{2}\right)_{5} \mathrm{NH}_{2}$ & $\mathrm{CF}_{3} \mathrm{C}_{6} \mathrm{~F}_{11}: \mathrm{CH}_{3} \mathrm{C}_{6} \mathrm{H}_{5}$ & $\begin{array}{l}\text { 56.9:43.1 } \\
(P=1.32)\end{array}$ & GLC & 26 \\
\hline$X-4^{c}$ & $\mathrm{R}_{\mathrm{f} 7} \mathrm{CH}_{2} \mathrm{NH}\left(\mathrm{CH}_{3}\right)$ & $\mathrm{CF}_{3} \mathrm{C}_{6} \mathrm{~F}_{11}: \mathrm{CH}_{3} \mathrm{C}_{6} \mathrm{H}_{5}$ & $\begin{array}{l}74.5: 25.5 \\
(\boldsymbol{P}=2.92)^{\mathrm{d}}\end{array}$ & GLC & 4a, 24 \\
\hline$X-5^{c}$ & $\mathrm{R}_{\mathrm{f} 7} \widehat{\mathrm{N}_{\mathrm{H}}}$ & $\mathrm{CF}_{3} \mathrm{C}_{6} \mathrm{~F}_{11}: \mathrm{CH}_{3} \mathrm{C}_{6} \mathrm{H}_{5}$ & $\begin{array}{l}30: 70 \\
(\boldsymbol{P}=0.42)^{\mathrm{d}}\end{array}$ & GLC & 24 \\
\hline$X-6^{c}$ & $\mathrm{R}_{\mathrm{f} 8}\left(\mathrm{CH}_{2}\right)_{3} \mathrm{NH}\left(\mathrm{CH}_{3}\right)$ & $\mathrm{CF}_{3} \mathrm{C}_{6} \mathrm{~F}_{11}: \mathrm{CH}_{3} \mathrm{C}_{6} \mathrm{H}_{5}$ & $\begin{array}{l}71: 29 \\
(\boldsymbol{P}=2.4)^{\mathrm{d}}\end{array}$ & GLC & $4 \mathrm{~b}$ \\
\hline $\mathrm{X}-7$ & {$\left[\mathrm{R}_{\mathrm{f} 8}\left(\mathrm{CH}_{2}\right)_{3}\right]_{2} \mathrm{NH}$} & $\mathrm{CF}_{3} \mathrm{C}_{6} \mathrm{~F}_{11}: \mathrm{CH}_{3} \mathrm{C}_{6} \mathrm{H}_{5}$ & $\begin{array}{l}96.5: 3.5 \\
(P=27.6)\end{array}$ & GLC & $4 b, 26$ \\
\hline $\mathrm{X}-8$ & {$\left[\mathrm{R}_{\mathrm{f} 8}\left(\mathrm{CH}_{2}\right)_{4}\right]_{2} \mathrm{NH}$} & $\mathrm{CF}_{3} \mathrm{C}_{6} \mathrm{~F}_{11}: \mathrm{CH}_{3} \mathrm{C}_{6} \mathrm{H}_{5}$ & $\begin{array}{l}\text { 95.1:4.9 } \\
(P=19.4)\end{array}$ & GLC & 26 \\
\hline X-9 & {$\left[\mathrm{R}_{\mathrm{f} 8}\left(\mathrm{CH}_{2}\right)_{5}\right]_{2} \mathrm{NH}$} & $\mathrm{CF}_{3} \mathrm{C}_{6} \mathrm{~F}_{11}: \mathrm{CH}_{3} \mathrm{C}_{6} \mathrm{H}_{5}$ & $\begin{array}{l}\text { 93.0:7.0 } \\
(P=13.3)\end{array}$ & GLC & 26 \\
\hline $\mathrm{X}-10^{\mathrm{c}}$ & $\mathrm{R}_{\mathrm{f} 7} \mathrm{CH}_{2} \mathrm{~N}\left(\mathrm{CH}_{3}\right)_{2}$ & $\mathrm{CF}_{3} \mathrm{C}_{6} \mathrm{~F}_{11}: \mathrm{CH}_{3} \mathrm{C}_{6} \mathrm{H}_{5}$ & $\begin{array}{l}82.2: 17.8 \\
(\boldsymbol{P}=4.62)^{\mathrm{d}}\end{array}$ & GLC & 24 \\
\hline$X-11^{c}$ & $\mathrm{R}_{\mathrm{f} 8}\left(\mathrm{CH}_{2}\right)_{3} \mathbf{N}\left(\mathrm{CH}_{3}\right)_{2}$ & $\mathrm{CF}_{3} \mathrm{C}_{6} \mathrm{~F}_{11}: \mathrm{CH}_{3} \mathrm{C}_{6} \mathrm{H}_{5}$ & $\begin{array}{l}79.8: 20.2 \\
(\boldsymbol{P}=3.94)^{\mathrm{d}}\end{array}$ & GLC & $4 \mathrm{~b}$ \\
\hline $\mathrm{X}-12^{\mathrm{c}}$ & {$\left[\mathrm{R}_{\mathrm{f} 8}\left(\mathrm{CH}_{2}\right)_{3}\right]_{2} \mathrm{~N}\left(\mathrm{CH}_{3}\right)$} & $\mathrm{CF}_{3} \mathrm{C}_{6} \mathrm{~F}_{11}: \mathrm{CH}_{3} \mathrm{C}_{6} \mathrm{H}_{5}$ & $\begin{array}{l}97.4: 2.6 \\
(P=37.7)^{\mathrm{d}}\end{array}$ & GLC & $4 \mathrm{~b}$ \\
\hline $\mathrm{X}-13$ & {$\left[\mathrm{R}_{\mathrm{f} 8}\left(\mathrm{CH}_{2}\right)_{3}\right]_{3} \mathrm{~N}$} & $\mathrm{CF}_{3} \mathrm{C}_{6} \mathrm{~F}_{11}: \mathrm{CH}_{3} \mathrm{C}_{6} \mathrm{H}_{5}$ & $\begin{array}{l}>99.7:<0.3 \\
(P>332)\end{array}$ & GLC & $4 \mathrm{~b}, 26$ \\
\hline$X-14$ & {$\left[\mathrm{R}_{\mathrm{f} 8}\left(\mathrm{CH}_{2}\right)_{4}\right]_{3} \mathrm{~N}$} & $\mathrm{CF}_{3} \mathrm{C}_{6} \mathrm{~F}_{11}: \mathrm{CH}_{3} \mathrm{C}_{6} \mathrm{H}_{5}$ & $\begin{array}{l}>99.7:<0.3 \\
(P>332)\end{array}$ & GLC & 26 \\
\hline $\mathrm{X}-15$ & $\begin{array}{l}{\left[\mathrm{R}_{\mathrm{f} 8}\left(\mathrm{CH}_{2}\right)_{5}\right]_{3} \mathbf{N}} \\
{\left[\left(\mathrm{R}_{\mathrm{f} 6}\left(\mathrm{CH}_{2}\right)_{2}\right)_{3} \mathrm{Si}\left(\mathrm{CH}_{2}\right)_{3}\right] \mathrm{NRR}^{\prime}}\end{array}$ & $\mathrm{CF}_{3} \mathrm{C}_{6} \mathrm{~F}_{11}: \mathrm{CH}_{3} \mathrm{C}_{6} \mathrm{H}_{5}$ & $\begin{array}{l}\text { 99.5:0.5 } \\
(P=199)\end{array}$ & GLC & 26 \\
\hline$X-16^{j}$ & $\mathrm{R}=\mathrm{H}, \mathrm{R}^{\prime}=$ & $\mathrm{C}_{6} \mathrm{~F}_{14}: \mathrm{CHCl}_{3}$ & $\begin{array}{l}42: 58 \\
(P=0.71)\end{array}$ & $\begin{array}{l}\text { gravimet or } \\
\text { HPLC }\end{array}$ & 15 \\
\hline$X-17 i$ & & $\mathrm{C}_{6} \mathrm{~F}_{14}: \mathrm{CH}_{3} \mathrm{CN}$ & $\begin{array}{l}27: 73 \\
(P=0.37)\end{array}$ & $\begin{array}{l}\text { gravimet or } \\
\text { HPLC }\end{array}$ & 15 \\
\hline $\mathrm{X}-18^{\mathrm{j}}$ & & $\mathrm{C}_{6} \mathrm{~F}_{14}: \mathrm{CH}_{3} \mathrm{OH}$ & $\begin{array}{l}67: 33 \\
(P=2.0)\end{array}$ & $\begin{array}{l}\text { gravimet or } \\
\text { HPLC }\end{array}$ & 15 \\
\hline$X-19 i$ & & $\mathrm{C}_{6} \mathrm{~F}_{14}: \mathrm{THF}$ & $\begin{array}{l}5: 95 \\
(P=0.05)\end{array}$ & $\begin{array}{l}\text { gravimet or } \\
\text { HPLC }\end{array}$ & 15 \\
\hline$X-20^{j}$ & $=\mathrm{CH}_{3}, \mathrm{R}$ & $\mathrm{C}_{6} \mathrm{~F}_{14}: \mathrm{CHCl}_{3}$ & $\begin{array}{l}17: 83 \\
(P=0.20)\end{array}$ & $\begin{array}{c}\text { gravimet or } \\
\text { HPLC }\end{array}$ & 15 \\
\hline $\mathrm{X}-21^{\mathrm{i}}$ & & $\mathrm{C}_{6} \mathrm{~F}_{14}: \mathrm{CH}_{3} \mathrm{CN}$ & $\begin{array}{l}44: 56 \\
(P=0.77)\end{array}$ & $\begin{array}{l}\text { gravimet or } \\
\text { HPLC }\end{array}$ & 15 \\
\hline
\end{tabular}


$76 \mid 6$ Partition Coefficients Involving Fluorous Solvents

Tab. 6-1. (continued)

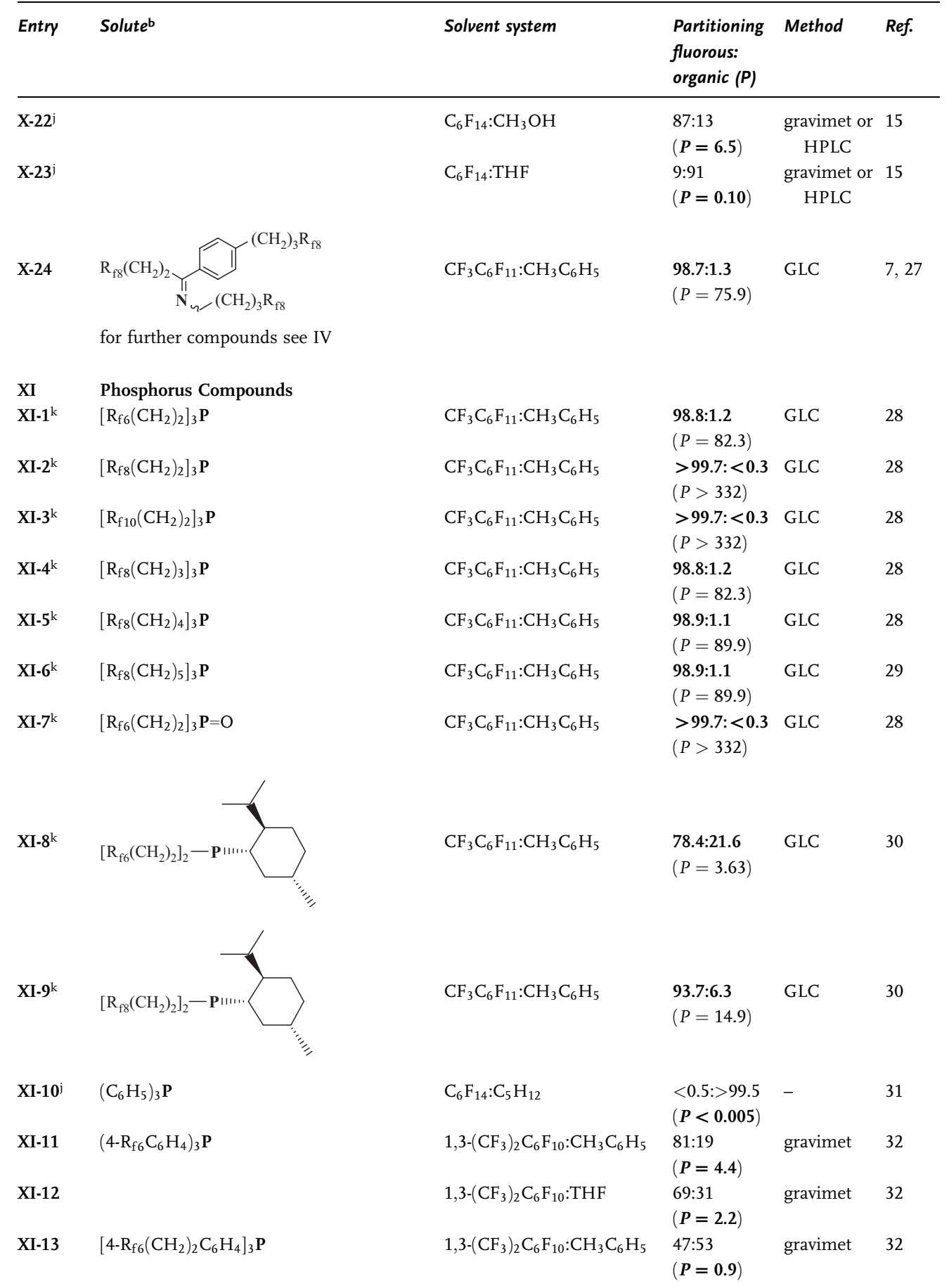


Tab. 6-1. (continued)

\begin{tabular}{|c|c|c|c|c|c|}
\hline Entry & Solute ${ }^{\mathrm{b}}$ & Solvent system & $\begin{array}{l}\text { Partitioning } \\
\text { fluorous: } \\
\text { organic (P) }\end{array}$ & Method & Ref. \\
\hline XI-14 & & $1,3-\left(\mathrm{CF}_{3}\right)_{2} \mathrm{C}_{6} \mathrm{~F}_{10}: \mathrm{THF}$ & $\begin{array}{l}17: 83 \\
(P=0.2)\end{array}$ & - & 32 \\
\hline XI-15g & & $\mathrm{C}_{6} \mathrm{~F}_{14}: \mathrm{CH}_{3} \mathrm{OH}$ & $\begin{array}{l}96.8: 3.2 \\
(P=30.03)\end{array}$ & HPLC & 33 \\
\hline XI-169 & & $\mathrm{C}_{6} \mathrm{~F}_{14}: \mathrm{THF}$ & $\begin{array}{l}7: 93 \\
(P=0.08)\end{array}$ & HPLC & 33 \\
\hline XI-17g & & $\mathrm{C}_{6} \mathrm{~F}_{14}: \mathrm{CH}_{3} \mathrm{C}_{6} \mathrm{H}_{5}$ & $\begin{array}{l}43: 57 \\
(P=0.75)\end{array}$ & HPLC & 33 \\
\hline $\mathrm{XI}-18^{\mathrm{k}}$ & {$\left[4-\mathrm{R}_{\mathrm{f} 6}\left(\mathrm{CH}_{2}\right)_{3} \mathrm{C}_{6} \mathrm{H}_{4}\right]_{3} \mathrm{P}$} & $\mathrm{CF}_{3} \mathrm{C}_{6} \mathrm{~F}_{11}: \mathrm{CH}_{3} \mathrm{C}_{6} \mathrm{H}_{5}$ & $\begin{array}{l}19.5: 80.5 \\
(P=0.242)\end{array}$ & GLC & 34 \\
\hline XI-19k & {$\left[4-\mathrm{R}_{\mathrm{f} 8}\left(\mathrm{CH}_{2}\right)_{3} \mathrm{C}_{6} \mathrm{H}_{4}\right]_{3} \mathbf{P}$} & $\mathrm{CF}_{3} \mathrm{C}_{6} \mathrm{~F}_{11}: \mathrm{CH}_{3} \mathrm{C}_{6} \mathrm{H}_{5}$ & $\begin{array}{l}\text { 66.6:33.4 } \\
(P=1.99)\end{array}$ & GLC & 34 \\
\hline $\mathrm{XI}-20^{\mathrm{c}}$ & {$\left[4-\mathrm{R}_{\mathrm{f} 8}\left(\mathrm{CH}_{2}\right)_{3} \mathrm{C}_{6} \mathrm{H}_{4}\right]_{3} \mathrm{P} \rightarrow \mathrm{BH}_{3}$} & $\mathrm{CF}_{3} \mathrm{C}_{6} \mathrm{~F}_{11}: \mathrm{CH}_{3} \mathrm{C}_{6} \mathrm{H}_{5}$ & $\begin{array}{l}37.3: 62.7 \\
(P=0.595)\end{array}$ & ${ }^{31} \mathrm{P}$ NMR & 17 \\
\hline $\mathrm{XI}-21^{\mathrm{c}}$ & {$\left[4-\left(\mathrm{R}_{\mathrm{f} 8} \mathrm{CH}_{2}\right)_{2} \mathrm{CH}\left(\mathrm{CH}_{2}\right)_{3} \mathrm{C}_{6} \mathrm{H}_{4}\right]_{3} \mathrm{P} \rightarrow \mathrm{BH}_{3}$} & $\mathrm{CF}_{3} \mathrm{C}_{6} \mathrm{~F}_{11}: \mathrm{CH}_{3} \mathrm{C}_{6} \mathrm{H}_{5}$ & $\begin{array}{l}\mathbf{9 6 . 6 : 3 . 4} \\
(P=28.4)\end{array}$ & ${ }^{19} \mathrm{~F}$ NMR & 17 \\
\hline $\mathrm{XI}-22^{\mathrm{g}}$ & {$\left[4-\mathrm{R}_{\mathrm{f}}\left(\mathrm{CH}_{2}\right)_{2} \mathrm{C}_{6} \mathrm{H}_{4}\right]_{2} \mathrm{PC}_{6} \mathrm{H}_{5}$} & $\mathrm{C}_{6} \mathrm{~F}_{14}: \mathrm{CH}_{3} \mathrm{OH}$ & $\begin{array}{l}\text { 65.0:35.0 } \\
(P=1.86)\end{array}$ & HPLC & 33 \\
\hline XI-23g & & $\mathrm{C}_{6} \mathrm{~F}_{14}: \mathrm{THF}$ & $\begin{array}{l}5: 95 \\
(P=\mathbf{0 . 0 5})\end{array}$ & HPLC & 33 \\
\hline XI-249 & & $\mathrm{C}_{6} \mathrm{~F}_{14}: \mathrm{CH}_{3} \mathrm{C}_{6} \mathrm{H}_{5}$ & $\begin{array}{l}5: 95 \\
(P=0.05)\end{array}$ & HPLC & 33 \\
\hline XI-25g & 4- $\mathrm{R}_{\mathrm{f} 6}\left(\mathrm{CH}_{2}\right)_{2} \mathrm{C}_{6} \mathrm{H}_{4} \mathrm{P}\left(\mathrm{C}_{6} \mathrm{H}_{5}\right)_{2}$ & $\mathrm{C}_{6} \mathrm{~F}_{14}: \mathrm{CH}_{3} \mathrm{OH}$ & $\begin{array}{l}11: 89 \\
(P=0.12)\end{array}$ & HPLC & 33 \\
\hline XI-26g & & $\mathrm{C}_{6} \mathrm{~F}_{14}: \mathrm{THF}$ & $\begin{array}{l}2: 98 \\
(P=0.02)\end{array}$ & HPLC & 33 \\
\hline XI-27g & & $\mathrm{C}_{6} \mathrm{~F}_{14}: \mathrm{CH}_{3} \mathrm{C}_{6} \mathrm{H}_{5}$ & $\begin{array}{l}5: 95 \\
(P=0.05)\end{array}$ & HPLC & 33 \\
\hline XI-28g & {$\left[4-\mathrm{R}_{\mathrm{f} 3}\left(\mathrm{CF}_{3}\right)_{2} \mathrm{CCH}_{2} \mathrm{C}_{6} \mathrm{H}_{4}\right]_{3} \mathbf{P}$} & $\mathrm{C}_{6} \mathrm{~F}_{14}: \mathrm{CH}_{3} \mathrm{OH}$ & $\begin{array}{l}94.9: 5.1 \\
(P=18.48)\end{array}$ & HPLC & 33 \\
\hline XI-29g & & $\mathrm{C}_{6} \mathrm{~F}_{14}: \mathrm{THF}$ & $\begin{array}{l}34: 66 \\
(P=0.51)\end{array}$ & HPLC & 33 \\
\hline XI-30g & & $\mathrm{C}_{6} \mathrm{~F}_{14}: \mathrm{CH}_{3} \mathrm{C}_{6} \mathrm{H}_{5}$ & $\begin{array}{l}87.2: 12.8 \\
(P=6.84)\end{array}$ & HPLC & 33 \\
\hline XI-31g & {$\left[4-\mathrm{R}_{\mathrm{f} 3}\left(\mathrm{CF}_{3}\right)_{2} \mathrm{CCH}_{2} \mathrm{C}_{6} \mathrm{H}_{4}\right]_{2} \mathrm{PC}_{6} \mathrm{H}_{5}$} & $\mathrm{C}_{6} \mathrm{~F}_{14}: \mathrm{CH}_{3} \mathrm{OH}$ & $\begin{array}{l}77.0: 23.0 \\
(\boldsymbol{P}=3.34)\end{array}$ & HPLC & 33 \\
\hline $\mathrm{XI}-32^{\mathrm{g}}$ & & $\mathrm{C}_{6} \mathrm{~F}_{14}: \mathrm{THF}$ & $\begin{array}{l}51.2: 48.8 \\
(P=1.05)\end{array}$ & HPLC & 33 \\
\hline XI-339 & & $\mathrm{C}_{6} \mathrm{~F}_{14}: \mathrm{CH}_{3} \mathrm{C}_{6} \mathrm{H}_{5}$ & $\begin{array}{l}15: 85 \\
(P=0.18)\end{array}$ & HPLC & 33 \\
\hline XI-349 & 4- $\mathrm{R}_{\mathrm{f} 3}\left(\mathrm{CF}_{3}\right)_{2} \mathrm{CCH}_{2} \mathrm{C}_{6} \mathrm{H}_{4} \mathrm{P}\left(\mathrm{C}_{6} \mathrm{H}_{5}\right)_{2}$ & $\mathrm{C}_{6} \mathrm{~F}_{14}: \mathrm{CH}_{3} \mathrm{OH}$ & $\begin{array}{l}8: 92 \\
(P=0.09)\end{array}$ & HPLC & 33 \\
\hline XI-35g & & $\mathrm{C}_{6} \mathrm{~F}_{14}: \mathrm{THF}$ & $\begin{array}{l}1: 99 \\
(P=0.01)\end{array}$ & HPLC & 33 \\
\hline $\mathrm{XI}-36^{\mathrm{g}}$ & & $\mathrm{C}_{6} \mathrm{~F}_{14}: \mathrm{CH}_{3} \mathrm{C}_{6} \mathrm{H}_{5}$ & $\begin{array}{l}11: 89 \\
(P=0.12)\end{array}$ & HPLC & 33 \\
\hline
\end{tabular}


$78 \mid 6$ Partition Coefficients Involving Fluorous Solvents

Tab. 6-1. (continued)

\begin{tabular}{|c|c|c|c|c|c|}
\hline Entry & Solute & Solvent system & $\begin{array}{l}\text { Partitioning } \\
\text { fluorous: } \\
\text { organic }(P)\end{array}$ & Method & Ref. \\
\hline $\mathrm{XI}-37^{\mathrm{i}}$ & {$\left[4-\left(\mathrm{CH}_{3}\right)_{3} \mathrm{SiC}_{6} \mathrm{H}_{4}\right]_{3} \mathbf{P}$} & $\mathrm{C}_{6} \mathrm{~F}_{14}: \mathrm{C}_{5} \mathrm{H}_{12}$ & $\begin{array}{l}<4:>96 \\
(P<0.044)\end{array}$ & - & 31 \\
\hline $\mathrm{XI}-38^{1}$ & & $\mathrm{C}_{6} \mathrm{~F}_{14}: n-\mathrm{C}_{6} \mathrm{H}_{14}$ & $\begin{array}{l}<1:>90 \\
(P<0.01)\end{array}$ & - & 31 \\
\hline XI-39m & $x=1, n=6$ & $\mathrm{CF}_{3} \mathrm{C}_{6} \mathrm{~F}_{11}: \mathrm{CH}_{3} \mathrm{C}_{6} \mathrm{H}_{5}$ & $\begin{array}{l}21: 79 \\
(P=0.26)\end{array}$ & ICP-AAS $^{n}$ & 35,36 \\
\hline $\mathrm{XI}-40^{\mathrm{m}}$ & & $\mathrm{CF}_{3} \mathrm{C}_{6} \mathrm{~F}_{11}: n-\mathrm{C}_{8} \mathrm{H}_{18}$ & $\begin{array}{l}52: 48 \\
(P=1.1)\end{array}$ & ICP-AAS & 36 \\
\hline $\mathrm{XI}-41^{\mathrm{m}}$ & & $\mathrm{CF}_{3} \mathrm{C}_{6} \mathrm{~F}_{11}: n-\mathrm{C}_{5} \mathrm{H}_{12}$ & $\begin{array}{l}60: 40 \\
(P=1.5)\end{array}$ & $\mathrm{ICP}^{-\mathrm{AAS}^{\mathrm{n}}}$ & 36 \\
\hline $\mathrm{XI}-42^{\mathrm{m}}$ & $x=1, n=8$ & $\mathrm{CF}_{3} \mathrm{C}_{6} \mathrm{~F}_{11}: \mathrm{CH}_{3} \mathrm{C}_{6} \mathrm{H}_{5}$ & $\begin{array}{l}69: 31 \\
(P=2.2)\end{array}$ & $\mathrm{ICP}^{-\mathrm{AAS}^{\mathrm{n}}}$ & 35,36 \\
\hline $\mathrm{XI}-43^{\mathrm{m}}$ & & $\mathrm{CF}_{3} \mathrm{C}_{6} \mathrm{~F}_{11}: n-\mathrm{C}_{8} \mathrm{H}_{18}$ & $\begin{array}{l}82: 18 \\
(P=4.6)\end{array}$ & ICP-AAS $^{n}$ & 36 \\
\hline $\mathrm{XI}-44^{\mathrm{m}}$ & & $\mathrm{CF}_{3} \mathrm{C}_{6} \mathrm{~F}_{11}: n-\mathrm{C}_{5} \mathrm{H}_{12}$ & $\begin{array}{l}69: 31 \\
(P=2.2)\end{array}$ & $\mathrm{ICP}^{-\mathrm{AAS}^{\mathrm{n}}}$ & 36 \\
\hline $\mathrm{XI}-45^{\mathrm{i}}$ & & $\mathrm{C}_{6} \mathrm{~F}_{14}: \mathrm{C}_{5} \mathrm{H}_{12}$ & $\begin{array}{l}12: 88 \\
(P=0.14)\end{array}$ & - & 31 \\
\hline $\mathrm{XI}-46^{1}$ & & $\mathrm{C}_{6} \mathrm{~F}_{14}: n-\mathrm{C}_{6} \mathrm{H}_{14}$ & $\begin{array}{l}43.5: 56.5 \\
(P=0.770)\end{array}$ & - & 31 \\
\hline $\mathrm{XI}-47^{\mathrm{m}}$ & $x=2, n=6$ & $\mathrm{CF}_{3} \mathrm{C}_{6} \mathrm{~F}_{11}: \mathrm{CH}_{3} \mathrm{C}_{6} \mathrm{H}_{5}$ & $\begin{array}{l}89: 11 \\
(P=7.8)\end{array}$ & ICP-AAS & 36 \\
\hline $\mathrm{XI}-48^{\mathrm{m}}$ & & $\mathrm{CF}_{3} \mathrm{C}_{6} \mathrm{~F}_{11}: n-\mathrm{C}_{8} \mathrm{H}_{18}$ & $\begin{array}{l}94: 6 \\
(P=17)\end{array}$ & ICP-AAS $^{n}$ & 36 \\
\hline $\mathrm{XI}-49^{\mathrm{m}}$ & & $\mathrm{CF}_{3} \mathrm{C}_{6} \mathrm{~F}_{11}: n-\mathrm{C}_{5} \mathrm{H}_{12}$ & $\begin{array}{l}85: 15 \\
(P=5.7)\end{array}$ & ICP-AAS & 36 \\
\hline $\mathrm{XI}-50^{\mathrm{m}}$ & $x=2, n=8$ & $\mathrm{CF}_{3} \mathrm{C}_{6} \mathrm{~F}_{11}: \mathrm{CH}_{3} \mathrm{C}_{6} \mathrm{H}_{5}$ & $\begin{array}{l}89: 11 \\
(P=7.8)\end{array}$ & ICP-AAS ${ }^{n}$ & 36 \\
\hline $\mathrm{XI}-51^{\mathrm{m}}$ & & $\mathrm{CF}_{3} \mathrm{C}_{6} \mathrm{~F}_{11}: n-\mathrm{C}_{8} \mathrm{H}_{18}$ & $\begin{array}{l}97: 3 \\
(P=28)\end{array}$ & ICP-AAS $^{n}$ & 36 \\
\hline $\mathrm{XI}-52^{\mathrm{m}}$ & & $\mathrm{CF}_{3} \mathrm{C}_{6} \mathrm{~F}_{11}: n-\mathrm{C}_{5} \mathrm{H}_{12}$ & $\begin{array}{l}90: 10 \\
(P=9.2)\end{array}$ & ICP-AAS & 36 \\
\hline XI- $53^{m}$ & $x=3, n=6$ & $\mathrm{CF}_{3} \mathrm{C}_{6} \mathrm{~F}_{11}: \mathrm{CH}_{3} \mathrm{C}_{6} \mathrm{H}_{5}$ & $\begin{array}{l}81: 19 \\
(P=4.3)\end{array}$ & ICP-AAS ${ }^{n}$ & 36 \\
\hline $\mathrm{XI}-54^{\mathrm{m}}$ & & $\mathrm{CF}_{3} \mathrm{C}_{6} \mathrm{~F}_{11}: n-\mathrm{C}_{8} \mathrm{H}_{18}$ & $\begin{array}{l}90: 10 \\
(P=9.4)\end{array}$ & $\mathrm{ICP}^{-\mathrm{AAS}^{\mathrm{n}}}$ & 36 \\
\hline $\mathrm{XI}-55^{\mathrm{m}}$ & & $\mathrm{CF}_{3} \mathrm{C}_{6} \mathrm{~F}_{11}: n-\mathrm{C}_{5} \mathrm{H}_{12}$ & $\begin{array}{l}94: 6 \\
(P=15)\end{array}$ & ICP-AAS $^{n}$ & 36 \\
\hline $\mathrm{XI}-56^{\mathrm{m}}$ & $x=3, n=8$ & $\mathrm{CF}_{3} \mathrm{C}_{6} \mathrm{~F}_{11}: \mathrm{CH}_{3} \mathrm{C}_{6} \mathrm{H}_{5}$ & $\begin{array}{l}68: 32 \\
(P=2.1)\end{array}$ & $\mathrm{ICP}^{-\mathrm{AAS}^{\mathrm{n}}}$ & 36 \\
\hline $\mathrm{XI}-57^{\mathrm{m}}$ & & $\mathrm{CF}_{3} \mathrm{C}_{6} \mathrm{~F}_{11}: n-\mathrm{C}_{8} \mathrm{H}_{18}$ & $\begin{array}{l}92: 8 \\
(P=12)\end{array}$ & $\mathrm{ICP}^{-\mathrm{AAS}^{\mathrm{n}}}$ & 36 \\
\hline
\end{tabular}


Tab. 6-1. (continued)

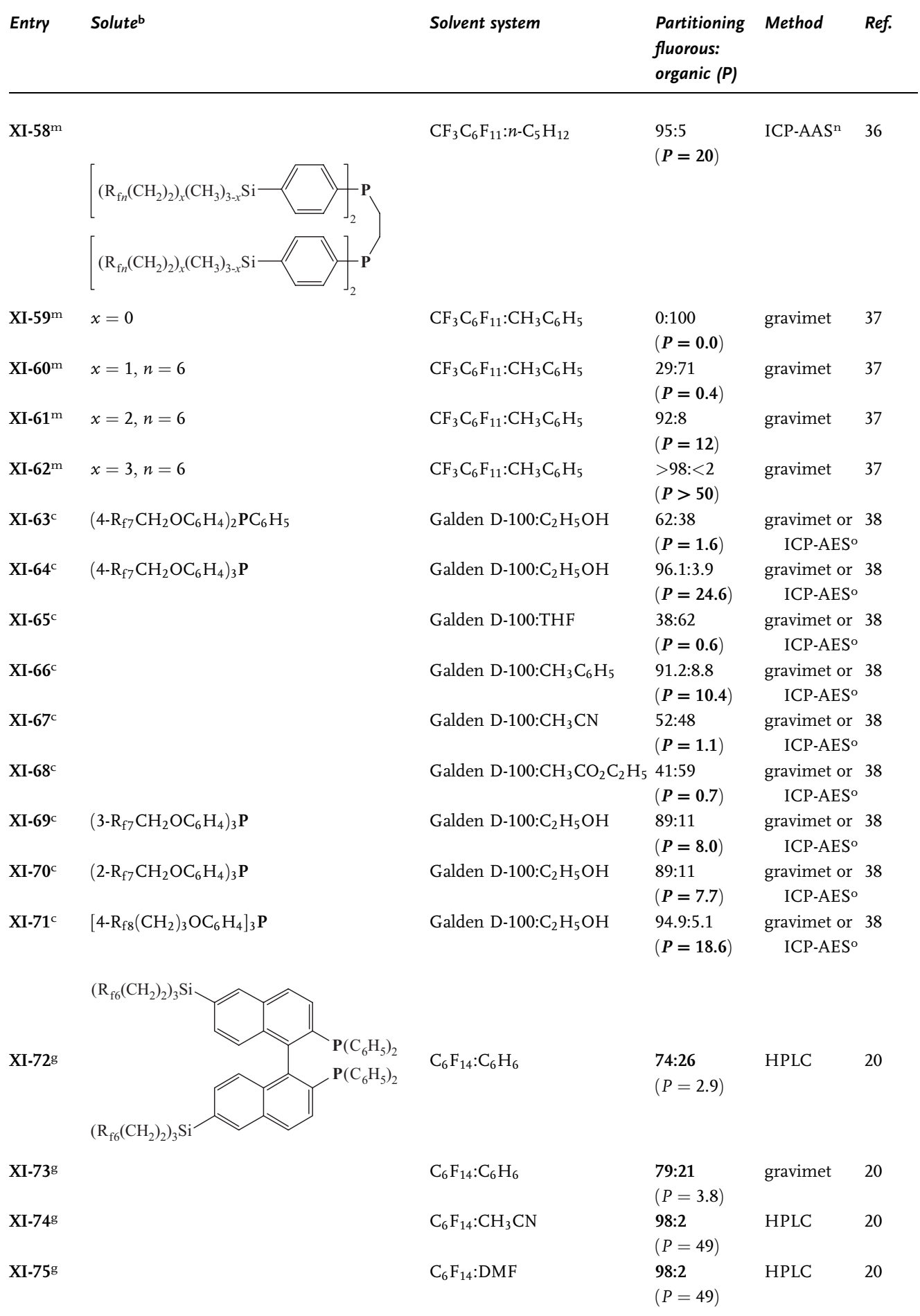


$80 \mid 6$ Partition Coefficients Involving Fluorous Solvents

Tab. 6-1. (continued)

\begin{tabular}{|c|c|c|c|c|c|}
\hline Entry & Solute ${ }^{\mathrm{b}}$ & Solvent system & $\begin{array}{l}\text { Partitioning } \\
\text { fluorous: } \\
\text { organic (P) }\end{array}$ & Method & Ref. \\
\hline XI-76g & & $\mathrm{C}_{6} \mathrm{~F}_{14}: \mathrm{CH}_{2} \mathrm{Cl}_{2}$ & $\begin{array}{l}76: 24 \\
(P=3.2)\end{array}$ & gravimet & 20 \\
\hline XI-77g & & $\mathrm{C}_{6} \mathrm{~F}_{14}: \mathrm{C}_{6} \mathrm{H}_{6}$ & $\begin{array}{l}\text { 90:10 } \\
(P=9.0)\end{array}$ & gravimet & 20 \\
\hline XI-78g & {$\left[2-\mathrm{R}_{\mathrm{f} 8}\left(\mathrm{CH}_{2}\right)_{2} \mathrm{C}_{6} \mathrm{H}_{4} \mathrm{O}\right]_{3} \mathrm{P}$} & $\mathrm{C}_{8} \mathrm{~F}_{17} \mathrm{H}: \mathrm{C}_{8} \mathrm{H}_{17} \mathrm{CH}=\mathrm{CH}_{2}$ & $\begin{array}{l}95: 5 \\
(P=19)\end{array}$ & GLC & 39 \\
\hline XI-79g & {$\left[4-\mathrm{R}_{\mathrm{f} 8}\left(\mathrm{CH}_{2}\right)_{2} \mathrm{C}_{6} \mathrm{H}_{4} \mathrm{O}\right]_{3} \mathrm{P}$} & $\mathrm{C}_{8} \mathrm{~F}_{17} \mathrm{H}: \mathrm{C}_{8} \mathrm{H}_{17} \mathrm{CH}=\mathrm{CH}_{2}$ & $\begin{array}{l}95: 5 \\
(P=19)\end{array}$ & GLC & 39 \\
\hline XI-80g & {$\left[2,4-\left(\mathrm{R}_{\mathrm{f} 8}\left(\mathrm{CH}_{2}\right)_{2}\right)_{2} \mathrm{C}_{6} \mathrm{H}_{3} \mathrm{O}\right]_{3} \mathbf{P}$} & $\mathrm{C}_{8} \mathrm{~F}_{17} \mathrm{H}: \mathrm{C}_{8} \mathrm{H}_{17} \mathrm{CH}=\mathrm{CH}_{2}$ & $\begin{array}{l}\text { 99:1 } \\
(P=99)\end{array}$ & GLC & 39 \\
\hline $\mathrm{XI}-81$ & {$\left[3,5-\left(\mathrm{R}_{\mathrm{f} 8}\left(\mathrm{CH}_{2}\right)_{3}\right)_{2} \mathrm{C}_{6} \mathrm{H}_{3} \mathrm{O}\right]_{3} \mathrm{P}$} & $\mathrm{CF}_{3} \mathrm{C}_{6} \mathrm{~F}_{11}: \mathrm{CH}_{3} \mathrm{C}_{6} \mathrm{H}_{5}$ & $\begin{array}{l}92: 8 \\
(P=12)\end{array}$ & $\begin{array}{l}{ }^{1} \mathrm{H} \text { and }{ }^{19} \mathrm{~F} \\
\text { NMR }\end{array}$ & 16 \\
\hline XII & Tin Compounds & & & & \\
\hline XII-19 & {$\left[\mathrm{CH}_{3}\left(\mathrm{CH}_{2}\right)_{3}\right]_{3} \mathrm{SnH}$} & $\mathrm{C}_{6} \mathrm{~F}_{14}: \mathrm{CH}_{3} \mathrm{CN}$ & $\begin{array}{l}<0.7:>99.3 \\
(P<0.007)\end{array}$ & gravimet & 40 \\
\hline XII-2g & & $\mathrm{C}_{6} \mathrm{~F}_{14}: \mathrm{C}_{6} \mathrm{H}_{6}$ & $\begin{array}{l}<0.3:>99.7 \\
(P<0.003)\end{array}$ & gravimet & 40 \\
\hline XII-3g & {$\left[\mathrm{R}_{\mathrm{f} 4}\left(\mathrm{CH}_{2}\right)_{2}\right]_{3} \mathrm{SnH}$} & $\mathrm{C}_{6} \mathrm{~F}_{14}: \mathrm{CH}_{3} \mathrm{CN}$ & $\begin{array}{l}85: 15 \\
(P=5.8)\end{array}$ & gravimet & 40 \\
\hline XII-4g & & $\mathrm{C}_{6} \mathrm{~F}_{14}: \mathrm{C}_{6} \mathrm{H}_{6}$ & $\begin{array}{l}91.3: 8.7 \\
(P=10.5)\end{array}$ & gravimet & 40 \\
\hline XII-5j & {$\left[\mathrm{R}_{\mathrm{f} 6}\left(\mathrm{CH}_{2}\right)_{2}\right]_{3} \mathrm{SnH}$} & $\mathrm{CF}_{3} \mathrm{C}_{6} \mathrm{~F}_{11}: \mathrm{C}_{6} \mathrm{H}_{6}$ & $\begin{array}{l}97.8: 2.2 \\
(P=44.5)\end{array}$ & gravimet & 41 \\
\hline XII-6j & & $\mathrm{CF}_{3} \mathrm{C}_{6} \mathrm{~F}_{11}: \mathrm{CH}_{3} \mathrm{OH}$ & $\begin{array}{l}\text { 97.0:3.0 } \\
(P=32.3)\end{array}$ & gravimet & 41 \\
\hline XII-7i & & $\mathrm{CF}_{3} \mathrm{C}_{6} \mathrm{~F}_{11}: \mathrm{CH}_{2} \mathrm{Cl}_{2}$ & $\begin{array}{l}95.3: 4.7 \\
(P=20.3)\end{array}$ & gravimet & 41 \\
\hline XII- $8^{j}$ & & $\mathrm{CF}_{3} \mathrm{C}_{6} \mathrm{~F}_{11}: \mathrm{CH}_{3} \mathrm{CO}_{2} \mathrm{C}_{2} \mathrm{H}_{5}$ & $\begin{array}{l}89.6: 10.4 \\
(P=8.62)\end{array}$ & gravimet & 41 \\
\hline XII-9i & & $\mathrm{CF}_{3} \mathrm{C}_{6} \mathrm{~F}_{11}: \mathrm{CHCl}_{3}$ & $\begin{array}{l}85.9: 14.1 \\
(P=6.09)\end{array}$ & gravimet & 41 \\
\hline XII-10g & & $\mathrm{C}_{6} \mathrm{~F}_{14}: \mathrm{CH}_{3} \mathrm{CN}$ & $\begin{array}{l}99.4: 0.6 \\
(P=160)\end{array}$ & gravimet & 40 \\
\hline XII-11g & & $\mathrm{C}_{6} \mathrm{~F}_{14}: \mathrm{C}_{6} \mathrm{H}_{6}$ & $\begin{array}{l}98: 2 \\
(P=45)\end{array}$ & gravimet & 40 \\
\hline $\mathrm{XII}-12^{\mathrm{g}}$ & {$\left[\mathrm{R}_{\mathrm{f} 4}\left(\mathrm{CH}_{2}\right)_{3}\right]_{3} \mathrm{SnH}$} & $\mathrm{C}_{6} \mathrm{~F}_{14}: \mathrm{CH}_{3} \mathrm{CN}$ & $\begin{array}{l}70: 30 \\
(P=2.3)\end{array}$ & gravimet & 40 \\
\hline XII-13g & & $\mathrm{C}_{6} \mathrm{~F}_{14}: \mathrm{C}_{6} \mathrm{H}_{6}$ & $\begin{array}{l}55: 45 \\
(P=1.2)\end{array}$ & gravimet & 40 \\
\hline
\end{tabular}


6.2 Literature Data $\mid 81$

Tab. 6-1. (continued)

\begin{tabular}{|c|c|c|c|c|c|}
\hline Entry & Solute ${ }^{\mathrm{b}}$ & Solvent system & $\begin{array}{l}\text { Partitioning } \\
\text { fluorous: } \\
\text { organic (P) }\end{array}$ & Method & Ref. \\
\hline XII-14g & {$\left[\mathrm{R}_{6}\left(\mathrm{CH}_{2}\right)_{3}\right]_{3} \mathrm{SnH}$} & $\mathrm{C}_{6} \mathrm{~F}_{14}: \mathrm{CH}_{3} \mathrm{CN}$ & $\begin{array}{l}86: 14 \\
(P=6.4)\end{array}$ & gravimet & 40 \\
\hline XII-15g & & $\mathrm{C}_{6} \mathrm{~F}_{14}: \mathrm{C}_{6} \mathrm{H}_{6}$ & $\begin{array}{l}90.9: 9.1 \\
(P=10.0)\end{array}$ & gravimet & 40 \\
\hline XII-16g & {$\left[\mathrm{R}_{\mathrm{f} 6}\left(\mathrm{CH}_{2}\right)_{2}\right] \mathbf{S n}\left(\mathrm{CH}_{3}\right)_{2} \mathrm{H}$} & $\mathrm{C}_{6} \mathrm{~F}_{14}: \mathrm{CH}_{3} \mathrm{CN}$ & $\begin{array}{l}71: 29 \\
(P=2.4)\end{array}$ & GLC & 40 \\
\hline XII-17g & & $\mathrm{C}_{6} \mathrm{~F}_{14}: \mathrm{C}_{6} \mathrm{H}_{6}$ & $\begin{array}{l}41: 59 \\
(P=0.7)\end{array}$ & GLC & 40 \\
\hline XII-18g & {$\left[\mathrm{R}_{\mathrm{f} 8}\left(\mathrm{CH}_{2}\right)_{2}\right] \mathbf{S n}\left(\mathrm{CH}_{3}\right)_{2} \mathrm{H}$} & $\mathrm{C}_{6} \mathrm{~F}_{14}: \mathrm{CH}_{3} \mathrm{CN}$ & $\begin{array}{l}93: 7 \\
(P=14)\end{array}$ & GLC & 40 \\
\hline XII-19g & & $\mathrm{C}_{6} \mathrm{~F}_{14}: \mathrm{C}_{6} \mathrm{H}_{6}$ & $\begin{array}{l}71: 29 \\
(P=2.5)\end{array}$ & GLC & 40 \\
\hline XII-20g & {$\left[\mathrm{R}_{\mathrm{f} 10}\left(\mathrm{CH}_{2}\right)_{2}\right] \mathbf{S n}\left(\mathrm{CH}_{3}\right)_{2} \mathrm{H}$} & $\mathrm{C}_{6} \mathrm{~F}_{14}: \mathrm{CH}_{3} \mathrm{CN}$ & $\begin{array}{l}98: 2 \\
(P=48)\end{array}$ & GLC & 40 \\
\hline XII-21g & & $\mathrm{C}_{6} \mathrm{~F}_{14}: \mathrm{C}_{6} \mathrm{H}_{6}$ & $\begin{array}{l}82: 18 \\
(P=4.7)\end{array}$ & GLC & 40 \\
\hline XII-22 & {$\left[\mathrm{R}_{\mathrm{f}}\left(\mathrm{CH}_{2}\right)_{2}\right]_{3} \mathrm{SnCH}_{2} \mathrm{CH}=\mathrm{CH}_{2}$} & $\mathrm{C}_{6} \mathrm{~F}_{14}: \mathrm{CH}_{3} \mathrm{CN}$ & $\begin{array}{l}86: 14 \\
(P=6)\end{array}$ & gravimet & 42 \\
\hline XII-23i & & $\mathrm{C}_{6} \mathrm{~F}_{14}: \mathrm{C}_{6} \mathrm{H}_{6}$ & $\begin{array}{l}90: 10 \\
(P=9)\end{array}$ & gravimet & 42 \\
\hline XII-24j & {$\left[\mathrm{R}_{\mathrm{f} 6}\left(\mathrm{CH}_{2}\right)_{2}\right]_{3} \mathrm{SnCH}_{2} \mathrm{CH}=\mathrm{CH}_{2}$} & $\mathrm{C}_{6} \mathrm{~F}_{14}: \mathrm{CH}_{3} \mathrm{CN}$ & $\begin{array}{l}99: 1 \\
(P=96)\end{array}$ & gravimet & 42 \\
\hline XII-25j & & $\mathrm{C}_{6} \mathrm{~F}_{14}: \mathrm{C}_{6} \mathrm{H}_{6}$ & $\begin{array}{l}98: 2 \\
(P=48)\end{array}$ & gravimet & 42 \\
\hline XII-26j & {$\left[\mathrm{R}_{\mathrm{f}_{4}}\left(\mathrm{CH}_{2}\right)_{3}\right]_{3} \mathrm{SnCH}_{2} \mathrm{CH}=\mathrm{CH}_{2}$} & $\mathrm{C}_{6} \mathrm{~F}_{14}: \mathrm{CH}_{3} \mathrm{CN}$ & $\begin{array}{l}93: 7 \\
(P=14)\end{array}$ & gravimet & 42 \\
\hline XII-27i & & $\mathrm{C}_{6} \mathrm{~F}_{14}: \mathrm{C}_{6} \mathrm{H}_{6}$ & $\begin{array}{l}80: 20 \\
(P=4)\end{array}$ & gravimet & 42 \\
\hline XII-28j & {$\left[\mathrm{R}_{\mathrm{f} 6}\left(\mathrm{CH}_{2}\right)_{3}\right]_{3} \mathrm{SnCH}_{2} \mathrm{CH}=\mathrm{CH}_{2}$} & $\mathrm{C}_{6} \mathrm{~F}_{14}: \mathrm{CH}_{3} \mathrm{CN}$ & $\begin{array}{l}98: 2 \\
(P=47)\end{array}$ & gravimet & 42 \\
\hline XII-29i & & $\mathrm{C}_{6} \mathrm{~F}_{14}: \mathrm{C}_{6} \mathrm{H}_{6}$ & $\begin{array}{l}96: 4 \\
(P=25)\end{array}$ & gravimet & 42 \\
\hline XII-30 & {$\left[\mathrm{R}_{\mathrm{f} 6}\left(\mathrm{CH}_{2}\right)_{2}\right]_{2} \mathrm{SnO}$} & $\mathrm{C}_{6} \mathrm{~F}_{14}: \mathrm{CH}_{2} \mathrm{Cl}_{2}$ & $\begin{array}{l}63.0: 37.0 \\
(P=1.70)\end{array}$ & - & 43 \\
\hline XII-31 & & $\mathrm{C}_{6} \mathrm{~F}_{14}: \mathrm{CH}_{3} \mathrm{CN}$ & $\begin{array}{l}22: 78 \\
(P=0.28)\end{array}$ & - & 43 \\
\hline XII-32 & & $\mathrm{C}_{6} \mathrm{~F}_{14}: \mathrm{CH}_{3} \mathrm{C}_{6} \mathrm{H}_{5}$ & $\begin{array}{l}\text { 65.5:34.5 } \\
(P=1.90)\end{array}$ & - & 43 \\
\hline XII-33i & {$\left[\mathrm{Cl}\left(\mathrm{R}_{\mathrm{f} 6}\left(\mathrm{CH}_{2}\right)_{2}\right)_{2} \mathrm{Sn}\right]_{2} \mathrm{O}$} & $\mathrm{C}_{6} \mathrm{~F}_{14}: \mathrm{CH}_{3} \mathrm{C}_{6} \mathrm{H}_{5}$ & $\begin{array}{l}99: 1 \\
(P=99)\end{array}$ & - & 44 \\
\hline XII-34 & & $\mathrm{C}_{6} \mathrm{~F}_{14}: \mathrm{CH}_{2} \mathrm{Cl}_{2}$ & $\begin{array}{l}99: 1 \\
(P=99)\end{array}$ & - & 44 \\
\hline XII-35j & & $\mathrm{C}_{6} \mathrm{~F}_{14}: \mathrm{CH}_{3} \mathrm{OH}$ & $\begin{array}{l}98: 2 \\
(P=49)\end{array}$ & - & 44 \\
\hline
\end{tabular}




\begin{tabular}{|c|c|c|c|c|c|}
\hline Entry & Solute & Solvent system & $\begin{array}{l}\text { Partitioning } \\
\text { fluorous: } \\
\text { organic (P) }\end{array}$ & Method & Ref. \\
\hline XIII & Silicon Compounds & & & & \\
\hline XIII-1 & $\mathrm{HSi}\left(\mathrm{CH}_{3}\right)_{2} \mathrm{C}_{6} \mathrm{H}_{5}$ & $\mathrm{CF}_{3} \mathrm{C}_{6} \mathrm{~F}_{11}: \mathrm{CH}_{3} \mathrm{C}_{6} \mathrm{H}_{5}$ & $\begin{array}{l}3.6: 96.4 \\
(P=0.037)\end{array}$ & GLC & $45 \mathrm{a}$ \\
\hline XIII-2m & $\mathrm{R}_{\mathrm{f} 6}\left(\mathrm{CH}_{2}\right)_{2} \mathrm{Si}\left(\mathrm{CH}_{3}\right)_{2} \mathrm{Cl}$ & $\mathrm{C}_{6} \mathrm{~F}_{14}: \mathrm{CH}_{3} \mathrm{C}_{6} \mathrm{H}_{5}$ & $\begin{array}{l}\text { 66:34 } \\
(P=1.9)\end{array}$ & $\mathrm{ICP}^{-\mathrm{AAS}^{\mathrm{n}}}$ & 46 \\
\hline XIII-3m & $\mathrm{R}_{\mathrm{f} 8}\left(\mathrm{CH}_{2}\right)_{2} \mathrm{Si}\left(\mathrm{CH}_{3}\right)_{2} \mathrm{Cl}$ & $\mathrm{C}_{6} \mathrm{~F}_{14}: \mathrm{CH}_{3} \mathrm{C}_{6} \mathrm{H}_{5}$ & $\begin{array}{l}83: 17 \\
(P=4.8)\end{array}$ & gravimet & 46 \\
\hline XIII-4 ${ }^{\mathrm{m}}$ & $\mathrm{R}_{\mathrm{f} 10}\left(\mathrm{CH}_{2}\right)_{2} \mathrm{Si}\left(\mathrm{CH}_{3}\right)_{2} \mathrm{Cl}$ & $\mathrm{C}_{6} \mathrm{~F}_{14}: \mathrm{CH}_{3} \mathrm{C}_{6} \mathrm{H}_{5}$ & $\begin{array}{l}95: 5 \\
(P=18)\end{array}$ & gravimet & 46 \\
\hline XIII-5m & $\mathrm{R}_{\mathrm{f} 6}\left(\mathrm{CH}_{2}\right)_{2} \mathrm{Si}\left(\mathrm{CH}_{3}\right) \mathrm{Cl}_{2}$ & $\mathrm{C}_{6} \mathrm{~F}_{14}: \mathrm{CH}_{3} \mathrm{C}_{6} \mathrm{H}_{5}$ & $\begin{array}{l}74: 26 \\
(P=2.8)\end{array}$ & ICP-AAS & 46 \\
\hline XIII-6m & $\mathrm{R}_{\mathrm{f} 6}\left(\mathrm{CH}_{2}\right)_{2} \mathrm{SiCl}_{3}$ & $\mathrm{C}_{6} \mathrm{~F}_{14}: \mathrm{CH}_{3} \mathrm{C}_{6} \mathrm{H}_{5}$ & $\begin{array}{l}83: 17 \\
(P=4.8)\end{array}$ & ICP-AAS & 46 \\
\hline XIII-7 & $-\mathrm{OSi}\left(\mathrm{CH}_{3}\right)_{2} \mathrm{C}_{6} \mathrm{H}_{5}$ & $\mathrm{CF}_{3} \mathrm{C}_{6} \mathrm{~F}_{11}: \mathrm{CH}_{3} \mathrm{C}_{6} \mathrm{H}_{5}$ & $\begin{array}{l}\mathbf{0 . 6 : 9 9 . 4} \\
(P=0.006)\end{array}$ & GLC & $45 \mathrm{a}$ \\
\hline XIII-8 & $-\mathrm{OSi}\left(\mathrm{CH}_{3}\right)_{2} \mathrm{C}_{6} \mathrm{H}_{5}$ & $\mathrm{CF}_{3} \mathrm{C}_{6} \mathrm{~F}_{11}: \mathrm{CH}_{3} \mathrm{C}_{6} \mathrm{H}_{5}$ & $\begin{array}{l}0.8: 99.2 \\
(P=0.008)\end{array}$ & GLC & $45 b$ \\
\hline & $\mathrm{ROSi}\left[\mathrm{CH}\left(\mathrm{CH}_{3}\right)_{2}\right]_{2}\left(\mathrm{CH}_{2}\right)_{2} \mathrm{R}^{\prime}$ & & & & \\
\hline XIII-9p & $\mathrm{R}=2$-(2-naphthyl)ethyl; $\mathrm{R}^{\prime}=\mathrm{R}_{\mathrm{f} 8}$ & $\mathrm{C}_{6} \mathrm{~F}_{14}: \mathrm{CH}_{3} \mathrm{OH}$ & $\begin{array}{l}48: 52 \\
(P=0.92)\end{array}$ & HPLC & 47 \\
\hline XIII-10p & & $\mathrm{C}_{6} \mathrm{~F}_{14}: \mathrm{C}_{2} \mathrm{H}_{5} \mathrm{OH}$ & $\begin{array}{l}48: 52 \\
(P=0.91)\end{array}$ & HPLC & 47 \\
\hline XIII-11p & & $\mathrm{C}_{6} \mathrm{~F}_{14}: \mathrm{CH}_{3} \mathrm{CN}$ & $\begin{array}{l}43: 57 \\
(P=0.74)\end{array}$ & HPLC & 47 \\
\hline XIII-12p & & $\mathrm{C}_{6} \mathrm{~F}_{14}: \mathrm{DMF}$ & $\begin{array}{l}28: 72 \\
(P=0.38)\end{array}$ & HPLC & 47 \\
\hline XIII-13p & & $\mathrm{C}_{6} \mathrm{~F}_{14}: \mathrm{CH}_{2} \mathrm{Cl}_{2}$ & $\begin{array}{l}12: 88 \\
(P=0.14)\end{array}$ & HPLC & 47 \\
\hline XIII-14P & & $\mathrm{C}_{6} \mathrm{~F}_{14}: \mathrm{THF}$ & $\begin{array}{l}3: 97 \\
(P=0.035)\end{array}$ & HPLC & 47 \\
\hline XIII-15p & $\mathrm{R}=2$-(2-naphthyl)ethyl; $\mathrm{R}^{\prime}=\mathrm{R}_{\mathrm{f} 10}$ & $\mathrm{C}_{6} \mathrm{~F}_{14}: \mathrm{CH}_{3} \mathrm{OH}$ & $\begin{array}{l}73: 27 \\
(P=2.7)\end{array}$ & HPLC & 47 \\
\hline XIII-16p & $\mathrm{R}=2-\left(\right.$ 2-naphthyl)ethyl; $\mathrm{R}^{\prime}=\mathrm{R}_{\mathrm{f} 6}$ & $\mathrm{C}_{6} \mathrm{~F}_{14}: \mathrm{CH}_{3} \mathrm{OH}$ & $\begin{array}{l}28: 72 \\
(P=0.39)\end{array}$ & HPLC & 47 \\
\hline XIII-17p & $\mathrm{R}=2$-(2-naphthyl)ethyl; $\mathrm{R}^{\prime}=\mathrm{R}_{\mathrm{f} 4}$ & $\mathrm{C}_{6} \mathrm{~F}_{14}: \mathrm{CH}_{3} \mathrm{OH}$ & $\begin{array}{l}11: 89 \\
(P=0.12)\end{array}$ & HPLC & 47 \\
\hline XIII-18p & $\mathrm{R}=(\mathrm{S})-(-)-1-\left(2-\right.$ naphthyl)ethyl; $\mathrm{R}^{\prime}=\mathrm{R}_{\mathrm{f} 8}$ & $\mathrm{C}_{6} \mathrm{~F}_{14}: \mathrm{CH}_{3} \mathrm{OH}$ & $\begin{array}{l}60: 40 \\
(P=1.5)\end{array}$ & HPLC & 47 \\
\hline XIII-19p & $\mathrm{R}=\mathrm{C}_{6} \mathrm{H}_{5} \mathrm{CH}=\mathrm{CHCH}_{2} ; \mathrm{R}^{\prime}=\mathrm{R}_{\mathrm{f} 8}$ & $\mathrm{C}_{6} \mathrm{~F}_{14}: \mathrm{CH}_{3} \mathrm{OH}$ & $\begin{array}{l}57: 43 \\
(P=1.3)\end{array}$ & HPLC & 47 \\
\hline XIII-20p & $\mathrm{R}=\mathrm{C}_{6} \mathrm{H}_{5}\left(\mathrm{CH}_{2}\right)_{6} ; \mathrm{R}^{\prime}=\mathrm{R}_{\mathrm{f} 8}$ & $\mathrm{C}_{6} \mathrm{~F}_{14}: \mathrm{CH}_{3} \mathrm{OH}$ & $\begin{array}{l}42: 58 \\
(P=0.72)\end{array}$ & HPLC & 47 \\
\hline
\end{tabular}


6.2 Literature Data $\mid 83$

Tab. 6-1. (continued)

\begin{tabular}{|c|c|c|c|c|c|}
\hline Entry & Solute ${ }^{\mathrm{b}}$ & Solvent system & $\begin{array}{l}\text { Partitioning } \\
\text { fluorous: } \\
\text { organic (P) }\end{array}$ & Method & Ref. \\
\hline XIII-21p & $\mathrm{R}=\mathrm{CH}_{3}\left(\mathrm{CH}_{2}\right)_{11} ; \mathrm{R}^{\prime}=\mathrm{R}_{\mathrm{f} 8}$ & $\mathrm{C}_{6} \mathrm{~F}_{14}: \mathrm{CH}_{3} \mathrm{OH}$ & $\begin{array}{l}89: 11 \\
(P=8.2)\end{array}$ & gravimet & 47 \\
\hline XIII-22p & $\mathrm{R}=$ cholestanyl; $\mathrm{R}^{\prime}=\mathrm{R}_{\mathrm{f} 8}$ & $\mathrm{C}_{6} \mathrm{~F}_{14}: \mathrm{CH}_{3} \mathrm{OH}$ & $\begin{array}{l}83: 17 \\
(P=5.0)\end{array}$ & HPLC & 47 \\
\hline XIII-23P & $\mathrm{R}=$ 2-adamantylethyl; $\mathrm{R}^{\prime}=\mathrm{R}_{\mathrm{f} 8}$ & $\mathrm{C}_{6} \mathrm{~F}_{14}: \mathrm{CH}_{3} \mathrm{OH}$ & $\begin{array}{l}85: 15 \\
(P=5.7)\end{array}$ & gravimet & 47 \\
\hline XIII-24p & $\mathrm{R}=2$-adamantylethyl; $\mathrm{R}^{\prime}=\mathrm{R}_{\mathrm{f} 6}$ & $\mathrm{C}_{6} \mathrm{~F}_{14}: \mathrm{CH}_{3} \mathrm{OH}$ & $\begin{array}{l}\text { 66:34 } \\
(P=1.9)\end{array}$ & gravimet & 47 \\
\hline XIII-25p & $\mathrm{R}=$ mappicine; $\mathrm{R}^{\prime}=\mathrm{R}_{\mathrm{f} 8}$ & $\mathrm{C}_{6} \mathrm{~F}_{14}: \mathrm{CH}_{3} \mathrm{OH}$ & $\begin{array}{l}10: 90 \\
(P=0.11)\end{array}$ & HPLC & 47 \\
\hline XIII-26m & $\begin{array}{l}\mathrm{R}_{\mathrm{f} 6}\left(\mathrm{CH}_{2}\right)_{2} \mathrm{Si}\left(\mathrm{OCH}_{3}\right)_{3} \\
\text { for further compounds see IV, VI, } \\
\quad \text { VII, X, XI, XV and XVI }\end{array}$ & $\mathrm{C}_{6} \mathrm{~F}_{14}: \mathrm{CH}_{3} \mathrm{C}_{6} \mathrm{H}_{5}$ & $\begin{array}{l}52: 48 \\
(P=1.1)\end{array}$ & gravimet & 46 \\
\hline XIV & Sulfur Compounds & & & & \\
\hline $\mathrm{XIV}-\mathbf{1}^{\mathrm{c}}$ & $\mathrm{HS}\left(\mathrm{CH}_{2}\right)_{2} \mathrm{C}(\mathrm{O}) \mathrm{OCH}_{2} \mathrm{CH}_{3}$ & $\mathrm{CF}_{3} \mathrm{C}_{6} \mathrm{~F}_{11}: \mathrm{CH}_{3} \mathrm{C}_{6} \mathrm{H}_{5}$ & $\begin{array}{l}0.5: 99.5 \\
(P=0.0054)^{\mathrm{d}}\end{array}$ & GLC & 24 \\
\hline $\mathrm{XIV}-2^{\mathrm{c}}$ & $\mathrm{R}_{\mathrm{f} 8}\left(\mathrm{CH}_{2}\right)_{3} \mathrm{SH}$ & $\mathrm{CF}_{3} \mathrm{C}_{6} \mathrm{~F}_{11}: \mathrm{CH}_{3} \mathrm{C}_{6} \mathrm{H}_{5}$ & $\begin{array}{l}56: 44 \\
(P=1.3)^{\mathrm{d}}\end{array}$ & GLC & $4 a$ \\
\hline XIV-3c & $\mathrm{R}_{\mathrm{f} 6} \mathrm{~S}\left(\mathrm{CH}_{2}\right)_{2} \mathrm{C}(\mathrm{O}) \mathrm{OCH}_{2} \mathrm{CH}_{3}$ & $\mathrm{CF}_{3} \mathrm{C}_{6} \mathrm{~F}_{11}: \mathrm{CH}_{3} \mathrm{C}_{6} \mathrm{H}_{5}$ & $\begin{array}{l}34: 66 \\
(P=0.51)^{\mathrm{d}}\end{array}$ & GLC & $4 a$ \\
\hline XIV-4c & $\mathrm{R}_{\mathrm{f}} \mathrm{S}\left(\mathrm{CH}_{2}\right)_{2} \mathrm{C}(\mathrm{O}) \mathrm{OCH}_{2} \mathrm{CH}_{3}$ & $\mathrm{CF}_{3} \mathrm{C}_{6} \mathrm{~F}_{11}: \mathrm{CH}_{3} \mathrm{C}_{6} \mathrm{H}_{5}$ & $\begin{array}{l}50: 50 \\
(P=1.0)^{\mathrm{d}}\end{array}$ & GLC & 24 \\
\hline XIV-5c & $\mathrm{CF}_{3} \mathrm{SC}_{6} \mathrm{H}_{5}$ & $\mathrm{CF}_{3} \mathrm{C}_{6} \mathrm{~F}_{11}: \mathrm{CH}_{3} \mathrm{C}_{6} \mathrm{H}_{5}$ & $\begin{array}{l}8: 92 \\
(P=0.086)^{\mathrm{d}}\end{array}$ & GLC & $4 a$ \\
\hline XIV-Gc & $\mathrm{CF}_{3} \mathrm{~S}\left(3-\mathrm{C}_{6} \mathrm{H}_{4} \mathrm{CF}_{3}\right)$ & $\mathrm{CF}_{3} \mathrm{C}_{6} \mathrm{~F}_{11}: \mathrm{CH}_{3} \mathrm{C}_{6} \mathrm{H}_{5}$ & $\begin{array}{l}17.1: 82.9 \\
(P=0.206)^{d}\end{array}$ & GLC & $4 a$ \\
\hline XIV-7c & $\mathrm{R}_{\mathrm{f} 8} \mathrm{SC}_{6} \mathrm{H}_{5}$ & $\mathrm{CF}_{3} \mathrm{C}_{6} \mathrm{~F}_{11}: \mathrm{CH}_{3} \mathrm{C}_{6} \mathrm{H}_{5}$ & $\begin{array}{l}64: 36 \\
(P=1.8)^{\mathrm{d}}\end{array}$ & GLC & $4 a$ \\
\hline XIV-8c & $\mathrm{R}_{\mathrm{f}} \mathrm{C}(\mathrm{O}) \mathrm{SCH}_{3}$ & $\mathrm{CF}_{3} \mathrm{C}_{6} \mathrm{~F}_{11}: \mathrm{CH}_{3} \mathrm{C}_{6} \mathrm{H}_{5}$ & $\begin{array}{l}76.1: 23.9 \\
(P=3.19)^{\mathrm{d}}\end{array}$ & GLC & 24 \\
\hline XIV-9c & $\mathrm{R}_{\mathrm{f} 7} \mathrm{C}(\mathrm{S}) \mathrm{OCH}_{3}$ & $\mathrm{CF}_{3} \mathrm{C}_{6} \mathrm{~F}_{11}: \mathrm{CH}_{3} \mathrm{C}_{6} \mathrm{H}_{5}$ & $\begin{array}{l}74.6: 25.4 \\
(P=2.94)^{\mathrm{d}}\end{array}$ & GLC & 24 \\
\hline XIV-10 & $\mathrm{R}_{\mathrm{f} 7} \mathrm{C}(\mathrm{S}) \mathrm{N}\left(\mathrm{CH}_{3}\right)_{2}$ & $\mathrm{CF}_{3} \mathrm{C}_{6} \mathrm{~F}_{11}: \mathrm{CH}_{3} \mathrm{C}_{6} \mathrm{H}_{5}$ & $\begin{array}{l}34: 66 \\
(P=0.52)^{\mathrm{d}}\end{array}$ & GLC & 24 \\
\hline XIV-11 ${ }^{c}$ & $\mathrm{R}_{\mathrm{f} 7}$ & $\mathrm{CF}_{3} \mathrm{C}_{6} \mathrm{~F}_{11}: \mathrm{CH}_{3} \mathrm{C}_{6} \mathrm{H}_{5}$ & $\begin{array}{l}17.4: 82.6 \\
(P=0.210)^{\mathrm{d}}\end{array}$ & GLC & 24 \\
\hline XIV-12c & & $\mathrm{CF}_{3} \mathrm{C}_{6} \mathrm{~F}_{11}: \mathrm{CH}_{3} \mathrm{C}_{6} \mathrm{H}_{5}$ & $\begin{array}{l}13.7: 86.3 \\
(P=0.159)^{d}\end{array}$ & GLC & 24 \\
\hline XIV-13 & {$\left[\mathrm{R}_{\mathrm{f} 8}\left(\mathrm{CH}_{2}\right)_{2}\right]_{2} \mathrm{~S}$} & $\mathrm{CF}_{3} \mathrm{C}_{6} \mathrm{~F}_{11}: \mathrm{CH}_{3} \mathrm{C}_{6} \mathrm{H}_{5}$ & $\begin{array}{l}\text { 98.7:1.3 } \\
(P=75.9)\end{array}$ & GLC & 48 \\
\hline
\end{tabular}


$84 \mid 6$ Partition Coefficients Involving Fluorous Solvents

Tab. 6-1. (continued)

\begin{tabular}{|c|c|c|c|c|c|}
\hline Entry & Solute & Solvent system & $\begin{array}{l}\text { Partitioning } \\
\text { fluorous: } \\
\text { organic (P) }\end{array}$ & Method & Ref. \\
\hline XIV-14 & {$\left[\mathrm{R}_{\mathrm{f} 8}\left(\mathrm{CH}_{2}\right)_{3}\right]_{2} \mathrm{~S}$} & $\mathrm{CF}_{3} \mathrm{C}_{6} \mathrm{~F}_{11}: \mathrm{CH}_{3} \mathrm{C}_{6} \mathrm{H}_{5}$ & $\begin{array}{l}\text { 96.6:3.4 } \\
(P=28.4)\end{array}$ & GLC & 48 \\
\hline XIV-15 & & $\mathrm{CF}_{3} \mathrm{C}_{6} \mathrm{~F}_{11}: \mathrm{CH}_{3} \mathrm{C}_{6} \mathrm{H}_{5}$ & $\begin{array}{l}99.5: 0.5 \\
(P=199)\end{array}$ & GLC & 7 \\
\hline
\end{tabular}

for further compounds see VI and X

XV Boron Compounds

(2) $\mathrm{CO}_{2}\left(\mathrm{CH}_{2}\right)_{3} \mathrm{R}_{\mathrm{fn}}$

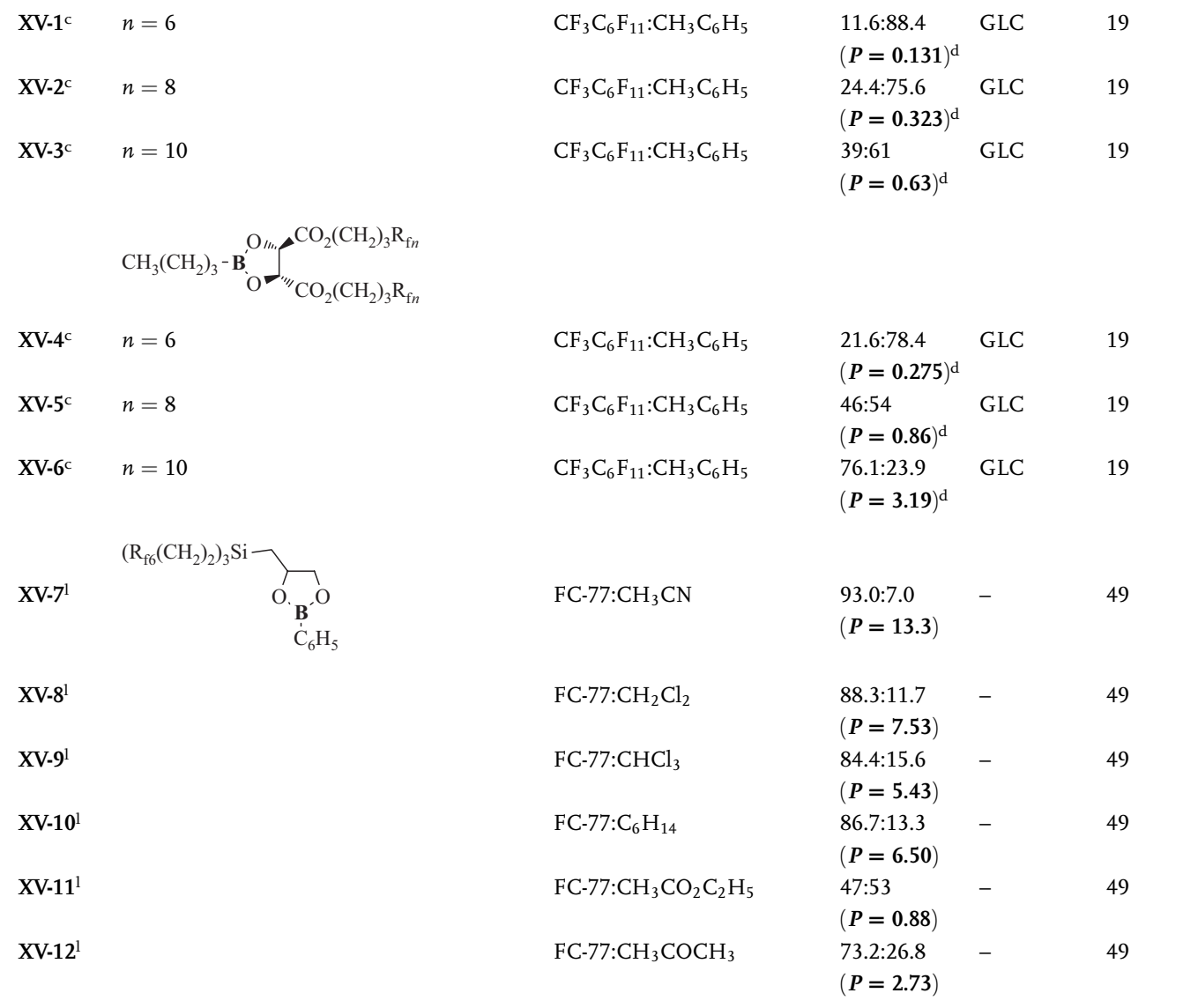


Tab. 6-1. (continued)

\begin{tabular}{|c|c|c|c|c|c|}
\hline Entry & Solute ${ }^{\mathrm{b}}$ & Solvent system & $\begin{array}{l}\text { Partitioning } \\
\text { fluorous: } \\
\text { organic (P) }\end{array}$ & Method & Ref. \\
\hline $\mathrm{XV}-13^{1}$ & & FC-77: $\mathrm{CH}_{3} \mathrm{C}_{6} \mathrm{H}_{5}$ & $\begin{array}{l}88.5: 11.5 \\
(P=7.69)\end{array}$ & - & 49 \\
\hline \multirow[t]{2}{*}{$\mathrm{XV}-14^{1}$} & & FC-77:THF & $\begin{array}{l}74.4: 25.6 \\
(P=2.91)\end{array}$ & - & 49 \\
\hline & for further compounds see XI and XVI & & & & \\
\hline XVI & Transition Metal Compounds & & & & \\
\hline XVI-1 ${ }^{\mathrm{j}}$ & $\mathbf{R h}_{2}\left(\mathrm{O}_{2} \mathrm{CR}_{\mathrm{f} 7}\right)_{4}$ & $\mathrm{CF}_{3} \mathrm{C}_{6} \mathrm{~F}_{11}: \mathrm{Et}_{2} \mathrm{O}$ & $\begin{array}{l}83: 17 \\
(P=4.9)\end{array}$ & gravimet & 50 \\
\hline$X V I-2^{j}$ & & $\mathrm{CF}_{3} \mathrm{C}_{6} \mathrm{~F}_{11}: \mathrm{CH}_{2} \mathrm{Cl}_{2}$ & $\begin{array}{l}94.9: 5.1 \\
(P=18.7)\end{array}$ & gravimet & 50 \\
\hline XVI-3i & & $\mathrm{CF}_{3} \mathrm{C}_{6} \mathrm{~F}_{11}: \mathrm{C}_{6} \mathrm{H}_{14}$ & $\begin{array}{l}96.7: 3.3 \\
(P=29.4)\end{array}$ & gravimet & 50 \\
\hline XVI-4 ${ }^{j}$ & & $\mathrm{CF}_{3} \mathrm{C}_{6} \mathrm{~F}_{11}: \mathrm{CH}_{3} \mathrm{C}_{6} \mathrm{H}_{5}$ & $\begin{array}{l}95.0: 5.0 \\
(P=18.9)\end{array}$ & gravimet & 50 \\
\hline XVI-5j & $\mathbf{R h}_{2}\left(\mathrm{O}_{2} \mathrm{CCH}_{2} \mathrm{R}_{\mathrm{f} 6}\right)_{4}$ & $\mathrm{CF}_{3} \mathrm{C}_{6} \mathrm{~F}_{11}: \mathrm{Et}_{2} \mathrm{O}$ & $\begin{array}{l}38: 62 \\
(P=0.6)\end{array}$ & gravimet & 50 \\
\hline XVI-6j & & $\mathrm{CF}_{3} \mathrm{C}_{6} \mathrm{~F}_{11}: \mathrm{CH}_{2} \mathrm{Cl}_{2}$ & $\begin{array}{l}89: 11 \\
(P=8.5)\end{array}$ & gravimet & 50 \\
\hline XVI-7i & & $\mathrm{CF}_{3} \mathrm{C}_{6} \mathrm{~F}_{11}: \mathrm{C}_{6} \mathrm{H}_{14}$ & $\begin{array}{l}96.6: 3.4 \\
(P=28.7)\end{array}$ & gravimet & 50 \\
\hline XVI-8i & & $\mathrm{CF}_{3} \mathrm{C}_{6} \mathrm{~F}_{11}: \mathrm{CH}_{3} \mathrm{C}_{6} \mathrm{H}_{5}$ & $\begin{array}{l}96.3: 3.7 \\
(P=25.7)\end{array}$ & gravimet & 50 \\
\hline XVI-9k & {$\left[\left(\mathrm{R}_{\mathrm{f} 6}\left(\mathrm{CH}_{2}\right)_{2}\right)_{3} \mathrm{P}\right]_{3} \mathrm{RhCl}$} & $\mathrm{CF}_{3} \mathrm{C}_{6} \mathrm{~F}_{11}: \mathrm{CH}_{3} \mathrm{C}_{6} \mathrm{H}_{5}$ & $\begin{array}{l}99.86: 0.14 \\
(P=713)\end{array}$ & ICP-AESo & 51 \\
\hline XVI-10 & {$\left[\left(\mathrm{R}_{\mathrm{f} 8}\left(\mathrm{CH}_{2}\right)_{2}\right)_{3} \mathrm{P}\right]_{3} \mathrm{RhCl}$} & $\mathrm{CF}_{3} \mathrm{C}_{6} \mathrm{~F}_{11}: \mathrm{CH}_{3} \mathrm{C}_{6} \mathrm{H}_{5}$ & $\begin{array}{l}99.88: 0.12 \\
(P=832)\end{array}$ & ICP-AES ${ }^{\circ}$ & 51 \\
\hline XVI-11 ${ }^{\mathrm{k}}$ & $\begin{array}{l}{\left[\left(\mathrm{R}_{\mathrm{f} 6}\left(\mathrm{CH}_{2}\right)_{2}\right)_{3} \mathrm{P}\right]_{3} \mathbf{R h}(\mathrm{H})(\mathrm{CO})} \\
\left(\mathrm{Ar}_{3} \mathrm{P}\right)_{3} \mathrm{RhCl}\end{array}$ & $\mathrm{CF}_{3} \mathrm{C}_{6} \mathrm{~F}_{11}: \mathrm{CH}_{3} \mathrm{C}_{6} \mathrm{H}_{5}$ & $\begin{array}{l}99.5: 0.5 \\
(P=190)^{\mathrm{d}}\end{array}$ & GLC & $4 a, 52 a$ \\
\hline $\mathrm{XVI}-12^{\mathrm{m}}$ & $\mathrm{Ar}=4-\mathrm{C}_{6} \mathrm{H}_{4} \mathrm{Si}\left(\mathrm{CH}_{3}\right)_{2}\left(\mathrm{CH}_{2}\right)_{2} \mathrm{R}_{\mathrm{f} 6}$ & $\mathrm{CF}_{3} \mathrm{C}_{6} \mathrm{~F}_{11}: n-\mathrm{C}_{8} \mathrm{H}_{18}$ & $\begin{array}{l}99.7: 0.3 \\
(P=293)\end{array}$ & ICP-AAS $^{n}$ & 35 \\
\hline XVI-13c & & $\mathrm{CF}_{3} \mathrm{C}_{6} \mathrm{~F}_{11}: n-\mathrm{C}_{8} \mathrm{H}_{18}$ & $\begin{array}{l}98.7: 1.3 \\
(P=76)\end{array}$ & ICP-AAS $^{n}$ & 35 \\
\hline XVI-14 ${ }^{\mathrm{m}}$ & $\mathrm{Ar}=4-\mathrm{C}_{6} \mathrm{H}_{4} \mathrm{Si}\left(\mathrm{CH}_{3}\right)_{2}\left(\mathrm{CH}_{2}\right)_{2} \mathrm{R}_{\mathrm{f} 8}$ & $\mathrm{CF}_{3} \mathrm{C}_{6} \mathrm{~F}_{11}: n-\mathrm{C}_{8} \mathrm{H}_{18}$ & $\begin{array}{l}99.9: 0.1 \\
(P=887)\end{array}$ & ${\mathrm{ICP}-A A S^{n}}^{n}$ & 35 \\
\hline XVI-15i & {$\left[\mathrm{R}_{\mathrm{f} 10}\left(\mathrm{CH}_{2}\right)_{2} \mathrm{C}_{5} \mathrm{H}_{4}\right] \mathbf{R h}(\mathrm{CO})_{2}$} & $\mathrm{CF}_{3} \mathrm{C}_{6} \mathrm{~F}_{11}: \mathrm{CH}_{3} \mathrm{C}_{6} \mathrm{H}_{5}$ & $\begin{array}{l}44: 56 \\
(P=0.8)\end{array}$ & ICP-AES ${ }^{\circ}$ & $52 \mathrm{a}$ \\
\hline XVI-16 ${ }^{\mathrm{i}}$ & $\begin{array}{l}{\left[\mathrm{R}_{\mathrm{f} 10}\left(\mathrm{CH}_{2}\right)_{2} \mathrm{C}_{5} \mathrm{H}_{4}\right] \mathbf{R h}(\mathrm{CO})\left[\mathrm{P}\left(\left(\mathrm{CH}_{2}\right)_{2} \mathrm{R}_{\mathrm{f} 6}\right)_{3}\right]} \\
{\left[\mathbf{R h}(\mathrm{COD})\left(\mathrm{Ar}_{2} \mathrm{P}\left(\mathrm{CH}_{2}\right)_{2} \mathrm{PAr}_{2}\right)\right]^{+} \mathrm{X}^{-}}\end{array}$ & $\mathrm{CF}_{3} \mathrm{C}_{6} \mathrm{~F}_{11}: \mathrm{CH}_{3} \mathrm{C}_{6} \mathrm{H}_{5}$ & $\begin{array}{l}96.7: 3.3 \\
(P=29)\end{array}$ & ICP-AES ${ }^{\circ}$ & $52 \mathrm{a}$ \\
\hline $\mathrm{XVI}-17^{\mathrm{m}}$ & $\begin{array}{l}\mathrm{Ar}=\mathrm{C}_{6} \mathrm{H}_{5} \\
\mathrm{X}^{-}=\left[\mathrm{B}\left(3,5-\mathrm{C}_{6} \mathrm{H}_{3}\left(\mathrm{R}_{\mathrm{f}}\right)_{2}\right)_{4}\right]^{-}\end{array}$ & $\mathrm{CF}_{3} \mathrm{C}_{6} \mathrm{~F}_{11}: \mathrm{THF}$ & $\begin{array}{l}<1:>99 \\
(P<0.01)\end{array}$ & ICP-AAS $^{n}$ & 53 \\
\hline XVI-18 & & $\mathrm{CF}_{3} \mathrm{C}_{6} \mathrm{~F}_{11}: \mathrm{CH}_{3} \mathrm{C}_{6} \mathrm{H}_{5}$ & $\begin{array}{l}41: 59 \\
(P=0.7)\end{array}$ & ICP-AAS $^{n}$ & 53 \\
\hline
\end{tabular}




\begin{tabular}{|c|c|c|c|c|c|}
\hline Entry & Solute ${ }^{\mathrm{b}}$ & Solvent system & $\begin{array}{l}\text { Partitioning } \\
\text { fluorous: } \\
\text { organic (P) }\end{array}$ & Method & Ref. \\
\hline XVI-19m & $\begin{array}{l}\mathrm{Ar}=4-\mathrm{C}_{6} \mathrm{H}_{4}\left[\mathrm{Si}\left(\mathrm{CH}_{3}\right)_{2}\left(\mathrm{CH}_{2}\right)_{2} \mathrm{R}_{\mathrm{f} 6}\right] \\
\mathrm{X}^{-}=\left[\mathrm{B}\left(3,5-\mathrm{C}_{6} \mathrm{H}_{3}\left(\mathrm{R}_{\mathrm{f}}\right)_{2}\right)_{4}\right]^{-}\end{array}$ & $\mathrm{CF}_{3} \mathrm{C}_{6} \mathrm{~F}_{11}: \mathrm{THF}$ & $\begin{array}{l}68: 32 \\
(P=2.1)\end{array}$ & $\mathrm{ICP}^{-\mathrm{AAS}^{\mathrm{n}}}$ & 53 \\
\hline XVI-20m & & $\mathrm{CF}_{3} \mathrm{C}_{6} \mathrm{~F}_{11}: \mathrm{CH}_{3} \mathrm{C}_{6} \mathrm{H}_{5}$ & $\begin{array}{l}97.1: 2.9 \\
(P=34)\end{array}$ & ICP-AASn $^{n}$ & 53 \\
\hline XVI-21 ${ }^{\mathrm{m}}$ & $\begin{array}{l}\mathrm{Ar}=4-\mathrm{C}_{6} \mathrm{H}_{4}\left[\mathrm{Si}\left(\mathrm{CH}_{3}\right)\left(\left(\mathrm{CH}_{2}\right)_{2} \mathrm{R}_{\mathrm{f}}\right)_{2}\right] \\
\mathrm{X}^{-}=\mathrm{BF}_{4}^{-}\end{array}$ & $\mathrm{CF}_{3} \mathrm{C}_{6} \mathrm{~F}_{11}: \mathrm{THF}$ & $\begin{array}{l}89: 11 \\
(P=8.1)\end{array}$ & ICP-AAS $^{n}$ & 53 \\
\hline $\mathrm{XVI}-22^{\mathrm{m}}$ & & $\mathrm{CF}_{3} \mathrm{C}_{6} \mathrm{~F}_{11}: \mathrm{CH}_{3} \mathrm{C}_{6} \mathrm{H}_{5}$ & $\begin{array}{l}98.4: 1.6 \\
(P=61)\end{array}$ & ICP-AAS $^{\mathrm{n}}$ & 53 \\
\hline XVI-23 ${ }^{\mathrm{m}}$ & $\begin{array}{l}\mathrm{Ar}=4-\mathrm{C}_{6} \mathrm{H}_{4}\left[\mathrm{Si}\left(\mathrm{CH}_{3}\right)\left(\left(\mathrm{CH}_{2}\right)_{2} \mathrm{R}_{\mathrm{f} 6}\right)_{2}\right] \\
\mathrm{X}^{-}=\left[\mathrm{B}\left(4-\mathrm{C}_{6} \mathrm{H}_{4} \mathrm{R}_{\mathrm{f} 6}\right)_{4}\right]^{-}\end{array}$ & $\mathrm{CF}_{3} \mathrm{C}_{6} \mathrm{~F}_{11}: \mathrm{THF}$ & $\begin{array}{l}86: 14 \\
(P=6.4)\end{array}$ & ICP-AAS $^{n}$ & 53 \\
\hline $\mathrm{XVI}-24^{\mathrm{m}}$ & & $\mathrm{CF}_{3} \mathrm{C}_{6} \mathrm{~F}_{11}: \mathrm{CH}_{3} \mathrm{C}_{6} \mathrm{H}_{5}$ & $\begin{array}{l}98.5: 1.5 \\
(P=65)\end{array}$ & ICP-AAS $^{n}$ & 53 \\
\hline XVI-25m & $\begin{array}{l}\mathrm{Ar}=4-\mathrm{C}_{6} \mathrm{H}_{4} \mathrm{Si}\left(\left(\mathrm{CH}_{2}\right)_{2} \mathrm{R}_{\mathrm{f} 6}\right)_{3} \\
\mathrm{X}^{-}=\mathrm{BF}_{4}^{-}\end{array}$ & $\mathrm{CF}_{3} \mathrm{C}_{6} \mathrm{~F}_{11}$ :THF & $\begin{array}{l}97.1: 2.9 \\
(P=34)\end{array}$ & ICP-AAS $^{n}$ & 53 \\
\hline XVI-26m & & $\mathrm{CF}_{3} \mathrm{C}_{6} \mathrm{~F}_{11}: \mathrm{CH}_{3} \mathrm{C}_{6} \mathrm{H}_{5}$ & $\begin{array}{l}98.5: 1.5 \\
(P=66)\end{array}$ & $\mathrm{ICP}^{-\mathrm{AAS}^{\mathrm{n}}}$ & 53 \\
\hline XVI-27i & {$\left[\mathrm{R}_{\mathrm{f} 10}\left(\mathrm{CH}_{2}\right)_{2} \mathrm{C}_{5} \mathrm{H}_{4}\right] \mathrm{Mn}(\mathrm{CO})_{3}$} & $\mathrm{CF}_{3} \mathrm{C}_{6} \mathrm{~F}_{11}: \mathrm{CH}_{3} \mathrm{C}_{6} \mathrm{H}_{5}$ & $\begin{array}{l}64: 36 \\
(P=1.8)\end{array}$ & IR & $52 b$ \\
\hline XVI-28g & {$\left[\left(\mathrm{CH}_{3}\left(\mathrm{CH}_{2}\right)_{7}\right)_{3} \mathrm{P}\right]_{2} \mathbf{I r}(\mathrm{Cl})(\mathrm{CO})$} & $\mathrm{CF}_{3} \mathrm{C}_{6} \mathrm{~F}_{11}: \mathrm{CH}_{3} \mathrm{C}_{6} \mathrm{H}_{5}$ & $\begin{array}{l}<0.3:>99.7 \\
(P<0.003)\end{array}$ & ${ }^{31} \mathrm{P}$ NMR & 54 \\
\hline XVI-29g & {$\left[\left(\mathrm{CH}_{3}\left(\mathrm{CH}_{2}\right)_{7}\right)_{3} \mathrm{P}\right]_{2} \mathbf{I r}(\mathrm{Cl})(\mathrm{CO})$} & $\mathrm{CF}_{3} \mathrm{C}_{6} \mathrm{~F}_{11}: \mathrm{THF}$ & $\begin{array}{l}<0.3:>99.7 \\
(P<0.003)\end{array}$ & ${ }^{31} \mathrm{P}$ NMR & 54 \\
\hline XVI-30g & {$\left[\left(\mathrm{R}_{\mathrm{f} 6}\left(\mathrm{CH}_{2}\right)_{2}\right)_{3} \mathrm{P}\right]_{2} \mathbf{I r}(\mathrm{Cl})(\mathrm{CO})$} & $\mathrm{CF}_{3} \mathrm{C}_{6} \mathrm{~F}_{11}: \mathrm{CH}_{3} \mathrm{C}_{6} \mathrm{H}_{5}$ & $\begin{array}{l}>99.7:<0.3 \\
(P>332)\end{array}$ & ${ }^{31} \mathrm{P}$ NMR & 54 \\
\hline XVI-319 & & $\mathrm{CF}_{3} \mathrm{C}_{6} \mathrm{~F}_{11}: \mathrm{CH}_{3} \mathrm{COCH}_{3}$ & $\begin{array}{l}>99.7:<0.3 \\
(P>332)\end{array}$ & ${ }^{31} \mathrm{P}$ NMR & 54 \\
\hline XVI-32g & & $\mathrm{CF}_{3} \mathrm{C}_{6} \mathrm{~F}_{11}: \mathrm{CH}_{2} \mathrm{Cl}_{2}$ & $\begin{array}{l}>99.7:<0.3 \\
(P>332)\end{array}$ & ${ }^{31} \mathrm{P}$ NMR & 54 \\
\hline XVI-33g & & $\mathrm{CF}_{3} \mathrm{C}_{6} \mathrm{~F}_{11}: \mathrm{THF}$ & $\begin{array}{l}>99.7:<0.3 \\
(P>332)\end{array}$ & ${ }^{31} \mathrm{P}$ NMR & 54 \\
\hline XVI-34 & $\begin{array}{l}{\left[\left(\mathrm{R}_{\mathrm{f} 6}\left(\mathrm{CH}_{2}\right)_{2}\right)_{3} \mathrm{P}\right]_{2} \mathrm{NiCl}_{2}} \\
\mathrm{NiCl}_{2}\left[\mathrm{Ar}_{2} \mathrm{P}\left(\mathrm{CH}_{2}\right)_{2} \mathrm{PAr}_{2}\right]\end{array}$ & $\mathrm{CF}_{3} \mathrm{C}_{6} \mathrm{~F}_{11}: \mathrm{CH}_{3} \mathrm{C}_{6} \mathrm{H}_{5}$ & $\begin{array}{l}98.8: 1.2 \\
(P=83)\end{array}$ & ICP-AES ${ }^{\circ}$ & $52 \mathrm{a}$ \\
\hline XVI-35 ${ }^{\mathrm{m}}$ & $\mathrm{Ar}=4-\mathrm{C}_{6} \mathrm{H}_{4} \mathrm{Si}\left(\mathrm{CH}_{3}\right)_{3}$ & $\mathrm{CF}_{3} \mathrm{C}_{6} \mathrm{~F}_{11}: \mathrm{CH}_{3} \mathrm{C}_{6} \mathrm{H}_{5}$ & $\begin{array}{l}0: 100 \\
(P=\mathbf{0 . 0})\end{array}$ & gravimet & 55 \\
\hline XVI-36 ${ }^{\mathrm{m}}$ & $\mathrm{Ar}=4-\mathrm{C}_{6} \mathrm{H}_{4}\left[\mathrm{Si}\left(\mathrm{CH}_{3}\right)_{2}\left(\mathrm{CH}_{2}\right)_{2} \mathrm{R}_{\mathrm{f} 6}\right]$ & $\mathrm{CF}_{3} \mathrm{C}_{6} \mathrm{~F}_{11}: \mathrm{CH}_{3} \mathrm{C}_{6} \mathrm{H}_{5}$ & $\begin{array}{l}\text { 62:38 } \\
(P=1.6)\end{array}$ & gravimet & 55 \\
\hline $\mathrm{XVI}-37^{\mathrm{m}}$ & $\mathrm{Ar}=4-\mathrm{C}_{6} \mathrm{H}_{4}\left[\mathrm{Si}\left(\mathrm{CH}_{3}\right)_{2}\left(\mathrm{CH}_{2}\right)_{2} \mathrm{R}_{\mathrm{f} 8}\right]$ & $\mathrm{CF}_{3} \mathrm{C}_{6} \mathrm{~F}_{11}: \mathrm{CH}_{3} \mathrm{C}_{6} \mathrm{H}_{5}$ & $\begin{array}{l}79: 21 \\
(P=3.7)\end{array}$ & gravimet & 55 \\
\hline XVI-38 & $\mathrm{Ar}=4-\mathrm{C}_{6} \mathrm{H}_{4}\left[\mathrm{Si}\left(\mathrm{CH}_{3}\right)\left(\left(\mathrm{CH}_{2}\right)_{2} \mathrm{R}_{\mathrm{f} 6}\right)_{2}\right]$ & $\mathrm{CF}_{3} \mathrm{C}_{6} \mathrm{~F}_{11}: \mathrm{CH}_{3} \mathrm{C}_{6} \mathrm{H}_{5}$ & $\begin{array}{l}89: 11 \\
(P=8)\end{array}$ & gravimet & 55 \\
\hline $\mathrm{XVI}-39^{\mathrm{m}}$ & $\mathrm{Ar}=4-\mathrm{C}_{6} \mathrm{H}_{4} \mathrm{Si}\left(\left(\mathrm{CH}_{2}\right)_{2} \mathrm{R}_{\mathrm{f} 6}\right)_{3}$ & $\mathrm{CF}_{3} \mathrm{C}_{6} \mathrm{~F}_{11}: \mathrm{CH}_{3} \mathrm{C}_{6} \mathrm{H}_{5}$ & $\begin{array}{l}95.8: 4.2 \\
(P=23)\end{array}$ & gravimet & 55 \\
\hline XVI-40 & $\mathrm{Fe}\left[\mathrm{C}_{5} \mathrm{H}_{4}\left(\mathrm{CH}_{2}\right)_{2} \mathrm{R}_{\mathrm{f} 10}\right]_{2}$ & $\mathrm{C}_{7} \mathrm{~F}_{16}: \mathrm{CH}_{3} \mathrm{C}_{6} \mathrm{H}_{5}$ & $\begin{array}{l}95.2: 4.8 \\
(P=20)\end{array}$ & gravimet & $52 \mathrm{~b}$ \\
\hline
\end{tabular}


6.2 Literature Data $\mid 87$

Tab. 6-1. (continued)

\begin{tabular}{|c|c|c|c|c|c|}
\hline Entry & Solute $e^{\mathrm{b}}$ & Solvent system & $\begin{array}{l}\text { Partitioning } \\
\text { fluorous: } \\
\text { organic }(P)\end{array}$ & Method & Ref. \\
\hline XVI-41c & $\mathrm{Fe}\left[\mathrm{C}_{5} \mathrm{H}_{3}\left(\left(\mathrm{CH}_{2}\right)_{2} \mathrm{R}_{\mathrm{f}}\right)_{2}\right]_{2} \mathrm{q}$ & $\mathrm{CF}_{3} \mathrm{C}_{6} \mathrm{~F}_{11}: \mathrm{CH}_{3} \mathrm{C}_{6} \mathrm{H}_{5}$ & $\begin{array}{l}\text { 90.9:9.1 } \\
(P=10)\end{array}$ & ICP-AAS $^{\mathrm{n}}$ & 6 \\
\hline XVI-42c & $\mathrm{Fe}\left[\mathrm{C}_{5} \mathrm{H}_{3}\left(\left(\mathrm{CH}_{2}\right)_{2} \mathrm{R}_{\mathrm{f} 6}\right)_{2}\right]_{2} \mathrm{q}$ & $\mathrm{CF}_{3} \mathrm{C}_{6} \mathrm{~F}_{11}: \mathrm{CH}_{3} \mathrm{C}_{6} \mathrm{H}_{5}$ & $\begin{array}{l}98.6: 1.4 \\
(P=72)\end{array}$ & ICP-AAS $^{n}$ & 6 \\
\hline XVI-43c & $\mathrm{Fe}\left[\mathrm{C}_{5} \mathrm{H}_{3}\left(\left(\mathrm{CH}_{2}\right)_{2} \mathrm{R}_{\mathrm{f} 4}\right)\left(\left(\mathrm{CH}_{2}\right)_{2} \mathrm{R}_{\mathrm{f} 6}\right)\right]_{2}^{\mathrm{q}}$ & $\mathrm{CF}_{3} \mathrm{C}_{6} \mathrm{~F}_{11}: \mathrm{CH}_{3} \mathrm{C}_{6} \mathrm{H}_{5}$ & $\begin{array}{l}94.4: 5.6 \\
(P=17)\end{array}$ & ICP-AAS $^{n}$ & 6 \\
\hline XVI-44c & $\mathrm{Fe}\left[\mathrm{C}_{5} \mathrm{H}_{3}\left(\left(\mathrm{CH}_{2}\right)_{2} \mathrm{R}_{\mathrm{f}}\right)\left(\left(\mathrm{CH}_{2}\right)_{2} \mathrm{R}_{\mathrm{f} 8}\right)\right]_{2} \mathrm{q}$ & $\mathrm{CF}_{3} \mathrm{C}_{6} \mathrm{~F}_{11}: \mathrm{CH}_{3} \mathrm{C}_{6} \mathrm{H}_{5}$ & $\begin{array}{l}95.7: 4.3 \\
(P=22)\end{array}$ & ICP-AAS $^{n}$ & 6 \\
\hline XVI-45c & $\mathrm{Fe}\left[\mathrm{C}_{5} \mathrm{H}_{3}\left(\left(\mathrm{CH}_{2}\right)_{2} \mathrm{R}_{\mathrm{f} 6}\right)\left(\left(\mathrm{CH}_{2}\right)_{2} \mathrm{R}_{\mathrm{f} 8}\right)\right]_{2} \mathrm{q}$ & $\mathrm{CF}_{3} \mathrm{C}_{6} \mathrm{~F}_{11}: \mathrm{CH}_{3} \mathrm{C}_{6} \mathrm{H}_{5}$ & $\begin{array}{l}95.7: 4.3 \\
(P=22)\end{array}$ & ICP-AAS $^{n}$ & 6 \\
\hline XVI-46g & {$\left[\mathrm{R}_{\mathrm{f} 6}\left(\mathrm{CH}_{2}\right)_{2} \mathrm{C}_{5} \mathrm{H}_{4}\right]_{2} \mathrm{ZrCl}_{2}$} & $\mathrm{CF}_{3} \mathrm{C}_{6} \mathrm{~F}_{11}: \mathrm{CH}_{3} \mathrm{C}_{6} \mathrm{H}_{5}$ & $\begin{array}{l}\text { 95.4:4.6 } \\
(P=20.7)\end{array}$ & gravimet & 56 \\
\hline XVI-47g & {$\left[\mathrm{R}_{\mathrm{f} 6}\left(\mathrm{CH}_{2}\right)_{2} \mathrm{C}_{5} \mathrm{H}_{4}\right]_{2} \mathrm{Zr}\left(\mathrm{CH}_{3}\right)_{2}$} & $\mathrm{CF}_{3} \mathrm{C}_{6} \mathrm{~F}_{11}: \mathrm{CH}_{3} \mathrm{C}_{6} \mathrm{H}_{5}$ & $\begin{array}{l}87.5: 12.5 \\
(P=7.00)\end{array}$ & gravimet & 56 \\
\hline XVI-48 & $\mathrm{R}_{\mathrm{f8}}\left(\mathrm{CH}_{2}\right)_{3} \mathrm{O} \cdot \mathrm{Pd} / 2$ & $\mathrm{CF}_{3} \mathrm{C}_{6} \mathrm{~F}_{11}: \mathrm{CH}_{3} \mathrm{C}_{6} \mathrm{H}_{5}$ & $\begin{array}{l}95.5: 4.5 \\
(P=21.2)\end{array}$ & HPLC & 7,27 \\
\hline XVI-49 & & $\mathrm{C}_{8} \mathrm{~F}_{17} \mathrm{Br}: \mathrm{DMF}$ & $\begin{array}{l}\text { 95.9:4.1 } \\
(P=23.4)\end{array}$ & HPLC & 7,27 \\
\hline XVI-50 & $\mathrm{R}_{\mathrm{f8}}\left(\mathrm{CH}_{2}\right)_{3}=\mathrm{R}_{\mathrm{f8}}\left(\mathrm{CH}_{2}\right)_{2}$ & $\mathrm{CF}_{3} \mathrm{C}_{6} \mathrm{~F}_{11}: \mathrm{CH}_{3} \mathrm{C}_{6} \mathrm{H}_{5}$ & $\begin{array}{l}\text { 90.7:9.3 } \\
(P=9.8)\end{array}$ & HPLC & 7 \\
\hline XVI-51 & & $\mathrm{C}_{8} \mathrm{~F}_{17} \mathrm{Br}: \mathrm{DMF}$ & $\begin{array}{l}91.4: 8.6 \\
(P=10.6)\end{array}$ & HPLC & 7 \\
\hline XVII & Supramolecular Complexes and Polymers & & & & \\
\hline XVII-1c & {$\left[\mathrm{R}_{\mathrm{f} 6}\left(\mathrm{CH}_{2}\right)_{2} \mathrm{NHCONHCH}\left(\mathrm{CH}_{3}\right)_{2}\right] \cdot \mathrm{HO}_{2} \mathrm{CR}_{\mathrm{f} 6}$} & $\mathrm{C}_{6} \mathrm{~F}_{14}: \mathrm{CH}_{2} \mathrm{Cl}_{2}$ & $\begin{array}{l}\mathbf{4 0 : 6 0} \\
(P=0.7)\end{array}$ & gravimet & 57 \\
\hline XVII-2c & {$\left[\left(\mathrm{CF}_{3} \mathrm{CH}_{2} \mathrm{NH}\right)_{2} \mathrm{CO}\right] \cdot \mathrm{HO}_{2} \mathrm{CR}_{\mathrm{f} 6}$} & $\mathrm{C}_{6} \mathrm{~F}_{14}: \mathrm{CH}_{2} \mathrm{Cl}_{2}$ & $\begin{array}{l}77: 23 \\
(P=3.4)\end{array}$ & gravimet & 57 \\
\hline XVII-3c & {$\left[\left(\mathrm{CF}_{3} \mathrm{CH}_{2} \mathrm{NH}\right)_{2} \mathrm{CO}\right] \cdot \mathrm{HO}_{2} \mathrm{CR}_{\mathrm{f} 17}$} & $\mathrm{C}_{6} \mathrm{~F}_{14}: \mathrm{CH}_{2} \mathrm{Cl}_{2}$ & $\begin{array}{l}83: 17 \\
(P=4.9)\end{array}$ & gravimet & 57 \\
\hline XVII-4c & {$\left[\left(\mathrm{R}_{\mathrm{f}}\left(\mathrm{CH}_{2}\right)_{2} \mathrm{NH}\right)_{2} \mathrm{CO}\right] \cdot \mathrm{HO}_{2} \mathrm{CR}_{\mathrm{f} 6}$} & $\mathrm{C}_{6} \mathrm{~F}_{14}: \mathrm{CH}_{2} \mathrm{Cl}_{2}$ & $\begin{array}{l}\mathbf{9 6 : 4} \\
(P=24)\end{array}$ & gravimet & 57 \\
\hline XVII-5c & {$\left[\left(\mathrm{R}_{\mathrm{f} 6}\left(\mathrm{CH}_{2}\right)_{2} \mathrm{NH}\right)_{2} \mathrm{CO}\right] \cdot \mathrm{HO}_{2} \mathrm{CR}_{\mathrm{f} 6}$} & $\mathrm{C}_{6} \mathrm{~F}_{14}: \mathrm{CH}_{2} \mathrm{Cl}_{2}$ & $\begin{array}{l}\text { 99:1 } \\
(P=99)\end{array}$ & gravimet & 57 \\
\hline XVII-6c & & $\mathrm{C}_{6} \mathrm{~F}_{14}: \mathrm{CHCl}_{3}$ & $\begin{array}{l}96: 4 \\
(P=24)\end{array}$ & gravimet & 57 \\
\hline
\end{tabular}


$88 \mid 6$ Partition Coefficients Involving Fluorous Solvents

Tab. 6-1. (continued)

\begin{tabular}{|c|c|c|c|c|c|}
\hline Entry & Solute ${ }^{\mathrm{b}}$ & Solvent system & $\begin{array}{l}\text { Partitioning } \\
\text { fluorous: } \\
\text { organic (P) }\end{array}$ & Method & Ref. \\
\hline XVII-7c & & $\mathrm{C}_{6} \mathrm{~F}_{14}: \mathrm{CH}_{3} \mathrm{CN}$ & $\begin{array}{l}\mathbf{9 0 : 1 0} \\
(P=9.0)\end{array}$ & gravimet & 57 \\
\hline XVII-8c & {$\left[\left(\mathrm{R}_{\mathrm{f} 6}\left(\mathrm{CH}_{2}\right)_{2} \mathrm{NH}\right)_{2} \mathrm{CO}\right] \cdot \mathrm{HO}_{2} \mathrm{CCH}_{2} \mathrm{R}_{\mathrm{f} 6}$} & $\mathrm{C}_{6} \mathrm{~F}_{14}: \mathrm{CH}_{2} \mathrm{Cl}_{2}$ & $\begin{array}{l}\mathbf{9 8 : 2} \\
(P=49)\end{array}$ & gravimet & 57 \\
\hline XVII-9c & {$\left[\left(\mathrm{R}_{\mathrm{f} 6}\left(\mathrm{CH}_{2}\right)_{2} \mathrm{NH}\right)_{2} \mathrm{CO}\right] \cdot \mathrm{HO}_{2} \mathrm{C}\left(\mathrm{CH}_{2}\right)_{2} \mathrm{R}_{\mathrm{f} 8}$} & $\mathrm{C}_{6} \mathrm{~F}_{14}: \mathrm{CH}_{2} \mathrm{Cl}_{2}$ & $\begin{array}{l}95: 5 \\
(P=19)\end{array}$ & gravimet & 57 \\
\hline XVII-10 & & $1,3-\left(\mathrm{CF}_{3}\right)_{2} \mathrm{C}_{6} \mathrm{~F}_{10}: \mathrm{CH}_{3} \mathrm{C}_{6} \mathrm{H}_{5}$ & $\begin{array}{l}96.4: 3.6 \\
(P=26.9)\end{array}$ & gravimet & 32 \\
\hline XVII-11 & 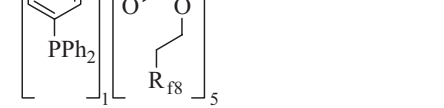 & $1,3-\left(\mathrm{CF}_{3}\right)_{2} \mathrm{C}_{6} \mathrm{~F}_{10}: \mathrm{THF}$ & $\begin{array}{l}96.5: 3.5 \\
(P=27.7)\end{array}$ & gravimet & 32 \\
\hline
\end{tabular}

a All measurements obtained at $24^{\circ} \mathrm{C}$ unless otherwise stated.

${ }^{\mathrm{b}} \mathrm{R}_{\mathrm{fn}}=\left(\mathrm{CF}_{2}\right)_{n-1} \mathrm{CF}_{3} .{ }^{\mathrm{c}} 25^{\circ} \mathrm{C}$. ${ }^{\mathrm{d}}$ Calculated from ln P. e Perfluorodecalin. ${ }^{\mathrm{f}}$ Mixture of isomers. ${ }^{\mathrm{g}}$ Ambient temperature implied. ${ }^{\mathrm{h}}-45^{\circ} \mathrm{C}$. ${ }^{\mathrm{i}} 20^{\circ} \mathrm{C}$. i T not given. ${ }^{\mathrm{k}} 27^{\circ} \mathrm{C} .{ }^{1} 10^{\circ} \mathrm{C}$. ${ }^{\mathrm{m}} 0{ }^{\circ} \mathrm{C}$. ${ }^{\mathrm{n}}$ Inductively coupled plasma atomic absorption spectrometry. ${ }^{\circ}$ Inductively coupled plasma - atomic emission spectrometry. ${ }^{\mathrm{P}} 23{ }^{\circ} \mathrm{C}$. ${ }^{9}$ Mixture of regioisomers.

\section{References}

1 Rocaboy, C; Rutherford, D.; Bennett, B. L.; Gladysz, J. A. J. Phys. Org. Chem. 2000, 13, 596.

2 Rutherford, D.; Juliette, J. J. J.; Rocaboy, C.; Horváth, I.; GLAdysz, J. A. Catalysis Today 1998, 42, 381.

3 Rutherford, D. unpublished results, University of Utah.

4 (a) Kiss, L. E.; Kövesdi, I.; RÁbai, J. J. Fluorine Chem. 2001, 108, 95.

(b) SZlÁvik, Z.; TÁRKÁNyi, G.; Gömöry, Á.; TARCZAY, G.; RÁbAI, J.

J. Fluorine Chem. 2001, 108, 7.

5 Solé-Violan, L.; Devaliez, B.; Postel, M.; Riess, J. G. New. J. Chem. 1993, 17, 581.

6 Kvícala, J.; Bríza, T.; Paleta, O.; Auerová, K.; Cermák, J. Tetrahedron 2002, 58, 3847.

7 Rocaboy, C.; Gladysz, J. A. New J. Chem. 2003, 27, 39.

8 Szlávik, Z.; TÁrkányi, G.; TARcZAy, G.; Gömöry, Á.; RÁbai, J. J. Fluorine Chem. 1999, 98, 83.

9 Takeuchi, S.; Nakamura, Y.; Ohgo, Y.; Curran, D. P. Tetrahedron Lett. 1998, 39, 8691.

10 Nakamura, Y.; Takeuchi, S.; OKumura, K.; Ohgo, Y. Tetrahedron 2001, 57, 5565.

11 Rábai, J.; Szabó, D.; Borbás, E. K.; Kövesi, I.; Kövesdi, I.; Csámpai, A.; Gömöry, Á.; Pashinnik, V. E.; Shermolovich, Y. G. J. Fluorine Chem. 2002, 114, 199.

12 Matsubara, H.; Yasuda, S.; Sugijama, H.; Ryu, I.; FujiI, Y.; Kita, K. Tetrahedron 2002, 58, 4071.

13 Pardo, J.; Cobas, A.; Guitián, E.; Castedo, L. Org. Lett. 2001, 3, 3711. 
Tab. 6-1 (continued)

14 Loiseau, J.; Fouquet, E.; Fish, R. H.; Vincent, J.-M.; Verlhac, J.-B. J. Fluorine Chem. 2001, 108, 195.

15 Linclau, B.; Singh, A. K.; Curran, D. P. J. Org. Chem. 1999, 64, 2835.

16 Le Stang, S.; Meier, R.; Rocaboy, C.; Gladysz, J. A. J. Fluorine Chem. 2003, 119, 141

17 Wende, M.; Seidel, F.; Gladysz, J. A. J. Fluorine Chem. 2003, 124, 45.

18 Rocaboy, C.; Gladysz, J. A. Chem. Eur. J. 2003, 9, 88.

19 Borbás, E. K., Masters Dissertation, Eötvös Loránd University Budapest, 2001

20 Nakamura, Y.; Takeuchi, S.; Ohgo, Y. J. Fluorine Chem. 2003, 120, 121.

21 Rocaboy, C.; Hampel, F.; Gladysz, J. A. J. Org. Chem. 2002, 67, 6863.

22 Annunziata, R.; Benagita, M.; Cinquini, M.; Cozzi, F.; Pozzi, G. Eur. J. Org. Chem. 2003, 1191.

23 Bayardon, J.; Sinou, D. Tetrahedron Lett. 2003, 44, 1449.

24 Kiss, L. E.; RÁbai, J.; Varga, L.; Kövesdi, I. Synlett 1998, 1243.

25 (a) Quici, S.; Cavazzini, M.; Ceragioli, S.; Montanari, F.; Pozzi, G. Tetrahedron Lett. 1999, 40, 3647. (b) MARKowiCZ, M. W.; Dembinski, R. Synthesis, in press.

26 Rocaboy, C.; Bauer, W.; Gladysz, J. A. Eur. J. Org. Chem. 2000, 2621.

27 Rocaboy, C.; Gladysz, J. A. Org. Lett. 2002, 4, 1993.

28 Alvey, L. J.; Rutherford, D.; Juliette, J. J. J.; Gladysz, J. A. J. Org. Chem. 1998, 63, 6302.

29 Alvey, L. J.; Meier, R.; Soós, T.; Bernatis, P.; Gladysz, J. A. Eur. J. Inorg. Chem. 2000, 1975.

30 Klose, A.; Gladysz, J. A. Tetrahedron: Asymmetry 1999, 10, 2665.

31 Richter, B.; Deelman, B.-J.; van Koten, G. J. Mol. Catal. A 1999, $145,317$.

32 Chen, W.; Xu, L.; Hu, Y.; Osuna, A. M. B.; XIAo, J. Tetrahedron 2002, 58, 3889.

33 Zhang, Q.; Luo, Z.; Curran, D. P. J. Org. Chem. 2000, 65, 8866.

34 Soós, T.; Bennett, B. L.; Rutherford, D.; Barthel-Rosa, L. P.; Gladysz, J. A. Organometallics 2001, 20, 3079.

35 Richter, B.; Spek, A. L.; van Koten, G.; Deelman, B.-J. J. Am. Chem. Soc. 2000, 122, 3945.

36 Richter, B.; de Wolf, E.; van Koten, G.; Deelman, B.-J. J. Org. Chem. 2000, 65, 3885.

37 de Wolf, E.; Richter, B.; Deelman, B.-J.; van Koten, G. J. Org. Chem. 2000, 65, 5424

38 Sinou, D.; Maillard, D.; Pozzi, G. Eur. J. Org. Chem. 2002, 269.

39 Mathivet, T.; Monflier, E.; Castanet, Y.; Mortreux, A.; Couturier, J.-L. Tetrahedron 2002, 58, 3877.

40 Curran, D. P.; Hadida, S.; Kim, S.-Y.; Luo, Z. J. Am. Chem. Soc. $1999,121,6607$.

41 Curran, D. P.; Hadida, S. J. Am. Chem. Soc. 1996, 118, 2531.

42 Curran, D. P.; Luo, Z.; Degenkolb, P. Bioorg. Med. Chem. Lett. $1998,8,2403$.

43 Bucher, B.; Curran, D. P. Tetrahedron Lett. 2000, 41, 9617.

44 Xiang, J.; Toyoshima, S.; Orita, A.; Otera, J. Angew. Chem., Int. Ed. 2001, 40, 3670 
Tab. 6-1 (continued)

45 (a) Dinh, L. V.; Gradysz, J. A. Tetrahedron Lett. 1999, 40, 8995. (b) DinH, L. V. unpublished results.

46 de Wolf, E.; Speets, E. A.; Deflman, B.-J.; van Koten, G. Organometallics 2001, 20,3686.

47 Nakamura, H.; Linclau, B.; Curran, D. P. J. Am. Chem. Soc. 2001, 123, 10119.

48 Rocaboy, C.; Gladysz, J. A. Tetrahedron 2002, 58, 4007.

49 Chen, D.; Qing, F.; Huang, Y. Org. Lett. 2002, 4, 1003.

50 Endres, A.; MaAs, G. J. Organomet. Chem. 2002, 643-644, 174.

51 Juliette, J. J. J.; Rutherford, D.; Horváth, I. T.; Gladysz, J. A. J. Am. Chem. Soc. 1999, 121, 2696.

52 (a) Herrera, V.; de Rege, P. J. F.; Horváth, I. T.; Husebo, T. L.; Hughes, R. P. Inorg. Chem. Commun. 1 1998, 197. (b) Hughes, R. P.; Trujillo, H. A. Organometallics 1996, 15, 286.

53 van den Broeke, J.; de Wolf, E.; Deelman, B.-J.; van Koten, G. Adv. Synth. Catal. 2003, 345, 625.

54 Guillevic, M.-A.; Rocaboy, C.; Arif, A. M.; Horváth, I. T.; Gladysz, J. A. Organometallics 1998, 17, 707.

55 de Wolf, E.; Mens, A. J. M.; Gijzeman, O. L. J.; van Lenthe, J. H.; Jenneskens, L. W.; Deelman, B.-J.; van Koten, G. Inorg. Chem. 2003, 42, 2115.

56 Ruwwe, J., Doctoral Dissertation, Westfälische Wilhelms Universität Münster, 1998.

57 Palomo, C.; Aizpurua, J. M.; Loinaz, I.; Fernandez-Berridi, M. J.; IRUSTA, L. Org. Lett. 2001, 3, 2361.

ences to other sections are given. The abbreviation $\mathrm{R}_{\mathrm{fn}}\left[\left(\mathrm{CF}_{2}\right)_{n-1} \mathrm{CF}_{3}\right]$ is used throughout, except for trifluoromethyl groups (formally $\mathrm{R}_{\mathrm{f} 1}$ ).

The partition coefficients are listed both as ratios normalized to 100 , and as $P$ values. As some rounding is necessary, the value originally reported in the literature is indicated in bold type. When a $P$ value has been calculated from an $\ln P$ or $f$ value, this is indicated by a footnote. All data from the authors' groups are believed to be correctly represented with respect to the number of significant digits.

GLC (gas-liquid chromatography), HPLC (high-performance liquid chromatography), and ICP-AAS/AES measurements are generally the most reliable. Gravimetric determinations where only a very small amount of the solute is present in one phase are subject to greater errors. Importantly, ICP methods give the total amount of a given element in a given phase. Hence, the decomposition of a solute to a species with a different partition coefficient (e.g., by oxidation), even to a small extent, can introduce error. Since GLC and HPLC assay a molecular characteristic, they are not subject to this problem.

Partition coefficients are temperature dependent, as illustrated by entries IV-9/IV-10 and XVI-12/XVI-13 of Table 6-1. The fluorous phase affinity of a fluorous solute is enhanced at lower temperature. However, most of the values in Table 6-1 were determined between 20 and $27^{\circ} \mathrm{C}$, as specified in the footnotes, and should not vary substantially within this range. In the most rigorous work, partition coefficients are determined over a range of concentrations and extrapolated to infinite dilution [1]. However, the concentrations used in the authors' experiments are close to those encountered in "real-life" fluorous/organic liquid/ liquid biphase separations, and the values for various classes of molecules are believed to have excellent cross-comparability. 
6.3

Trends with Respect to Functional Groups

6.3.1

Non-Aromatic Hydrocarbons

Entries I-1 to I-6 in Table 6-1 give partition coefficients $\left(\mathrm{CF}_{3} \mathrm{C}_{6} \mathrm{~F}_{11} /\right.$ toluene $)$ for $n$-alkanes (decane through hexadecane), and entries II-1 to II-6 give data for the corresponding terminal alkenes. The alkanes, although very non-polar, show high affinities for the toluene phase. These increase monotonically with alkane size (5.4:94.6 for decane to 1.1:98.9 for hexadecane). This is in accord with the general trends for absolute solubilities in fluorous solvents, as discussed in Chapter 3.5. Non-fluorous solutes are thought to occupy cavities, and smaller guests are always better accommodated.

The $n$-alkenes have slightly higher toluene phase affinities, consistent with their slightly greater polarities. A comparable monotonic size trend is found (4.8:95.2 for 1-decene to 0.9:99.1 for 1-hexadecene). When the side-chain of 1-decene is perfluorinated to give $\mathrm{R}_{\mathrm{f} 8} \mathrm{CH}=\mathrm{CH}_{2}$, the partition coefficient nearly reverses, to 93.5:6.5 (entry II-7). A variety of hemifluorinated disubstituted alkenes, $\mathrm{R}_{\mathrm{fn}} \mathrm{CH}=\mathrm{CH}\left(\mathrm{CH}_{2}\right)_{m} \mathrm{CH}_{3}$, have been analyzed (entries II-8 to II-43). As would be expected, they exhibit intermediate fluorophilicities.

6.3.2

Non-Aromatic Monofunctional Compounds

Entries III-1 and III-2 give partition coefficients $\left(\mathrm{CF}_{3} \mathrm{C}_{6} \mathrm{~F}_{11} /\right.$ toluene $)$ for the ketones cyclohexanone and 2-cyclohexen-1-one - species more polar than alkanes and alkenes but somewhat "smaller" than specific examples discussed above. Their toluene phase affinities are also high (2.2:97.8 and 1.7:98.3). The toluene phase affinity of cyclohexanol (entry IV-1, 1.6:98.4) is higher than that of cyclohexanone, and the corresponding dimethylphenyl silyl ether is higher still (entry XIII-8, 0.8:99.2).

Section IV of Table 6-1 contains several simple fluorous alcohols. The short-chain species in entries IV-2 and IV-3 show poor fluorophilicities [7]. As the perfluoroalkyl segment lengthens in the series $\mathrm{R}_{\mathrm{f} 6}\left(\mathrm{CH}_{2}\right)_{3} \mathrm{OH}, \mathrm{R}_{\mathrm{f} 8}\left(\mathrm{CH}_{2}\right)_{3} \mathrm{OH}$, and $\mathrm{R}_{\mathrm{f} 10}\left(\mathrm{CH}_{2}\right)_{3} \mathrm{OH}$ (entries IV-5, IV-7, IV-8), the fluorous phase affinities increase from $44: 56$ to $64: 36$ to $80.5: 19.5\left(\mathrm{CF}_{3} \mathrm{C}_{6} \mathrm{~F}_{11} /\right.$ toluene). As would be expected, when a methylene group is removed from the first two compounds, the fluorophilicities also increase (52:48 and 73.5:26.5; entries IV-4 and IV-6). Similar trends are found with all other functional groups in Table 6-1.

The thiol $\mathrm{R}_{\mathrm{f} 8}\left(\mathrm{CH}_{2}\right)_{3} \mathrm{SH}$ (entry XIV-2), iodide $\mathrm{R}_{\mathrm{f} 8}\left(\mathrm{CH}_{2}\right)_{3} \mathrm{I}$ (entry IX-4), and primary amine $\mathrm{R}_{\mathrm{f} 8}\left(\mathrm{CH}_{2}\right)_{3} \mathrm{NH}_{2}$ (entry X-1) exhibit $\mathrm{CF}_{3} \mathrm{C}_{6} \mathrm{~F}_{11}$ /toluene partition coefficients (56:44, 50.7:49.3, and 70.0:30.0) roughly comparable to that of the corresponding alcohol (64:36). Thus, more than one $\mathrm{R}_{\mathrm{f8}}$-containing ponytail is clearly needed to achieve higher fluorophilicities with simple monofunctional organic compounds.

The effect of the number of ponytails is clearly seen in amines of the formula $\left[\mathrm{R}_{\mathrm{f} 8}\left(\mathrm{CH}_{2}\right)_{3}\right]_{x} \mathrm{NH}_{3-x}$ (entries X-1, X-7, and X-13). As $x$ increases from one to three, the fluorous phase affinities increase monotonically from 70.0:30.0 to 96.5:3.5 to the point where no GLC-detectable concentration in toluene remains $(>99.7:<0.3)$. Thus, $\left[\mathrm{R}_{\mathrm{f} 8}\left(\mathrm{CH}_{2}\right)_{3}\right]_{3} \mathrm{~N}$ repre- 
sents a highly immobilized fluorous base. When the number of the methylene groups in each ponytail is increased to five, a small amount of the amine can again be detected in the toluene phase (99.5:0.5; entry X-15).

Similar trends are observed with fluorous trialkylphosphines. Here, the lengths of the $\mathrm{R}_{\mathrm{fn}}$ as well as the $\left(\mathrm{CH}_{2}\right)_{m}$ segments have been varied (entries XI-1 to XI-6), and very high fluorous phase affinities can be achieved $\left\{>99.7:<0.3\right.$ for $\left[\mathrm{R}_{\mathrm{f} 8}\left(\mathrm{CH}_{2}\right)_{2}\right]_{3} \mathrm{P}$ and $\left.\left[\mathrm{R}_{\mathrm{f} 10}\left(\mathrm{CH}_{2}\right)_{2}\right]_{3} \mathrm{P}\right\}$. Where comparisons are possible, fluorophilicities are slightly lower than for analogous amines. Counter-intuitively from a polarity standpoint, oxidation to a phosphine oxide slightly increases the fluorous phase affinity (entry XI-7 vs. XI-1).

As shown in Section XII of Table 6-1, appropriately designed trialkyltin hydrides can also be highly fluorophilic. Although the solvent systems used are slightly different, partition coefficients for the compounds $\left[\mathrm{R}_{\mathrm{fn}}\left(\mathrm{CH}_{2}\right)_{2}\right]_{3} \mathrm{SnH}$ are comparable to those of the corresponding phosphines (entries XII-5 and XII-11 for $n=6 ; n=10$ is still more fluorophilic but due to solubility limitations an accurate value could not be determined) [8]. Another triply-branched system is the tertiary alcohol $\left[\left(\mathrm{R}_{\mathrm{f} 6}\left(\mathrm{CH}_{2}\right)_{2}\right]_{3} \mathrm{COH}\right.$. The $\mathrm{C}_{6} \mathrm{~F}_{14} / \mathrm{THF}$ partition coefficient is 90.9:9.1 at ambient temperature or 95.8:4.2 at $-45^{\circ} \mathrm{C}$ (entries IV-9 and IV-10).

Thioethers, which can only accommodate two ponytails around the central heteroatom, possess fluorous phase affinities slightly lower than those of comparable amines and phosphines. For example, the $\mathrm{CF}_{3} \mathrm{C}_{6} \mathrm{~F}_{11}$ /toluene partition coefficients for $\left[\mathrm{R}_{\mathrm{f} 8}\left(\mathrm{CH}_{2}\right)_{2}\right]_{2} \mathrm{~S}$ and $\left[\mathrm{R}_{\mathrm{f} 8}\left(\mathrm{CH}_{2}\right)_{3}\right]_{2} \mathrm{~S}$ are 98.7:1.3 and 96.6:3.4 (entries XIV-13 and XIV-14).

\section{3 .3}

\section{Simple Monoarenes}

Data for simple arenes are collected in Section VII of Table 6-1, and the $\mathrm{CF}_{3} \mathrm{C}_{6} \mathrm{~F}_{11} /$ toluene partition coefficients can be analyzed as follows. Both pentafluorobenzene and hexafluorobenzene preferentially partition into toluene (22.4:77.6 and 28.0:72.0; entries VII-2 and VII-3), consistent with their non-fluorous nature as described in Chapter 3.2.2. Benzene exhibits an even greater toluene phase affinity (6:94; entry VII-1). However, the introduction of a single ponytail of formula $\mathrm{R}_{\mathrm{f} 8}\left(\mathrm{CH}_{2}\right)_{3}$ evens the playing field, and a partition coefficient of 49.5:50.5 is obtained (entry VII-18). This value is similar to those obtained when an $\mathrm{R}_{\mathrm{f} 8}\left(\mathrm{CH}_{2}\right)_{3}$ moiety is capped with an iodide or thiol. The compound $\mathrm{R}_{\mathrm{f} 8} \mathrm{C}_{6} \mathrm{H}_{5}$ (entry VII-16), which lacks methylene spacers, is more fluorophilic still (77.5:22.5), but the electronic properties of the arene ring are strongly perturbed.

As shown in entries VII-43, VII-45, and VII-46, benzenes with two ponytails of formula $\mathrm{R}_{\mathrm{f} 8}\left(\mathrm{CH}_{2}\right)_{3}$ exhibit appreciable fluorophilicities, with partition coefficients of 91.2:8.8 to 90.7:9.3. The substitution pattern has little influence. As seen with other compounds above, when the perfluoroalkyl segment of the ponytail is shortened, the fluorous phase affinity decreases [73.7:26.3 for $\mathrm{R}_{\mathrm{f} 6}\left(\mathrm{CH}_{2}\right)_{3}$; entry VII-42], and when it is lengthened the fluorous phase affinity increases [97.4:2.6 for $\mathrm{R}_{\mathrm{f} 10}\left(\mathrm{CH}_{2}\right)_{3}$; entry VII-44]. Importantly, benzenes with three ponytails of formula $\mathrm{R}_{\mathrm{f} 8}\left(\mathrm{CH}_{2}\right)_{3}$ partition (within detection limits) completely into $\mathrm{CF}_{3} \mathrm{C}_{6} \mathrm{~F}_{11}$, at least when arrayed in a 1,3,5-pattern (entry VII-61).

Entries VII-49 through to VII-54, VII-62, and VII-63 feature monoiodide derivatives of some of the preceding fluorous benzenes. In all cases, the fluorophilicities decrease. Only for entry VII-62, a triply ponytailed compound, is a highly biased $\mathrm{CF}_{3} \mathrm{C}_{6} \mathrm{~F}_{11} /$ toluene partition 
coefficient maintained (98.0:2.0). When the more polar non-fluorous solvent methanol is employed, relative fluorous phase affinities increase. Regardless, it is clear that with monofunctional benzenes, at least three ponytails of the formula $\mathrm{R}_{\mathrm{f} 8}\left(\mathrm{CH}_{2}\right)_{3}$ are required for high degrees of fluorous phase immobilization. Entries III-4, IV-11, and VI-7 - a benzaldehyde, benzyl alcohol, and methyl benzoate - show that two ponytails of formula $R_{f 8}$ (i.e., without spacers $)$ are essentially as effective $\left(\mathrm{CF}_{3} \mathrm{C}_{6} \mathrm{~F}_{11}\right.$ / toluene partition coefficients 98.6:1.4, 97.4:2.6 and 98.8:1.2).

\subsection{4}

Triarylphosphines

These points are further illustrated by some of the para-substituted fluorous triarylphosphines in Section XI of Table 6-1. With one ponytail of the formula $\mathrm{R}_{\mathrm{f} 6}\left(\mathrm{CH}_{2}\right)_{3}$ per ring, the $\mathrm{CF}_{3} \mathrm{C}_{6} \mathrm{~F}_{11}$ /toluene partition coefficient is 19.5:80.5 (entry XI-18). When the perfluoroalkyl segment is lengthened, giving $\mathrm{R}_{\mathrm{f} 8}\left(\mathrm{CH}_{2}\right)_{3}$, the fluorophilicity increases (66.6:33.4; entry XI-19). When the methylene segment is shortened, giving $\mathrm{R}_{\mathrm{f} 6}\left(\mathrm{CH}_{2}\right)_{2}$, the fluorophilicity should also increase. Accordingly, the $\mathrm{C}_{6} \mathrm{~F}_{14} /$ toluene partition coefficient is 43:57 (entry XI-17). When toluene is replaced by the much more polar solvent methanol, the fluorous phase affinity becomes much higher (96.8:3.2, entry XI-15).

Entries XI-30 and XI-28 provide analogous data for a phosphine with a single methylene spacer and a branched $\mathrm{R}_{\mathrm{f} 6}$ moiety. In $\mathrm{C}_{6} \mathrm{~F}_{14}$ /toluene, the fluorous phase affinity is lower (87.2:12.8), but in $\mathrm{C}_{6} \mathrm{~F}_{14}$ /methanol, slightly higher (94.9:5.1). When the phenyl groups bear only $p-\mathrm{R}_{\mathrm{f} 6}$ substituents (no methylene spacers), the partition coefficient in $1,3-\left(\mathrm{CF}_{3}\right)_{2} \mathrm{C}_{6} \mathrm{~F}_{10}$ / toluene is $81: 19$ (entry XI-11).

It has proved problematic to attach additional ponytails directly onto the aryl moieties of fluorous triarylphosphines. Thus, there are no readily available compounds of the formula $\left\{\left[\mathrm{R}_{\mathrm{fn}}\left(\mathrm{CH}_{2}\right)_{m}\right]_{x} \mathrm{C}_{6} \mathrm{H}_{5-x}\right\}_{3} \mathrm{P}(m>0)$ with truly high fluorous phase affinities. However, a clever way around this dilemma has been developed [9]. Namely, silicon-based ponytails of the formula $\left[\mathrm{R}_{\mathrm{fn}}\left(\mathrm{CH}_{2}\right)_{2}\right]_{x} \mathrm{Si}\left(\mathrm{CH}_{3}\right)_{3-x}$ have been used as anchors for as many as three $\mathrm{R}_{\mathrm{f} 6}$ or $\mathrm{R}_{\mathrm{f} 8}$ groups per ring [10]. As summarized in entries XI-47 through to XI-58, this gives triarylphosphines with much higher fluorous phase affinities (up to 95:5 for $x=3$ and $n=8$ in $\left.\mathrm{CF}_{3} \mathrm{C}_{6} \mathrm{~F}_{11} / n-\mathrm{C}_{5} \mathrm{H}_{12}\right)$. When the exact values are carefully analyzed, some non-monotonic trends are obvious, and this point is treated further below.

Note that the analogous dppe derivative in entry XI-62, which contains two fluorous arenes on each phosphorus atom, gives a $\mathrm{CF}_{3} \mathrm{C}_{6} \mathrm{~F}_{11} /$ toluene partition coefficient of $>98:<2$. The related BINAP derivative in entries XI-72 through to XI-75 features only a single fluorous arene on each phosphorus atom, and the $\mathrm{C}_{6} \mathrm{~F}_{14} / \mathrm{C}_{6} \mathrm{H}_{6}$ partition coefficient is only 74:26 (HPLC).

6.3 .5

Pyridines

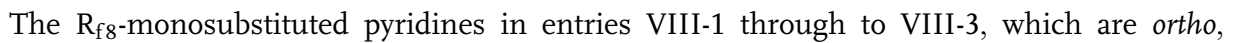
meta, and para isomers, give $\mathrm{CF}_{3} \mathrm{C}_{6} \mathrm{~F}_{11}$ /toluene partition coefficients in the narrow range of 
63:37 to 71:29. The $\mathrm{R}_{\mathrm{f} 8}\left(\mathrm{CH}_{2}\right)_{3}$-disubstituted pyridine in entry VIII-5 is rigorously comparable to the benzenoid analog in entry VII-45 ( $\mathrm{N} / \mathrm{CH}$ exchange), and gives an essentially identical partition coefficient (90.4:9.6 vs. 90.7:9.3). Hence, the polar pyridine nitrogen has little influence. Entry VIII-7 shows that three ponytails of the formula $\mathrm{R}_{\mathrm{f} 8}\left(\mathrm{CH}_{2}\right)_{2}$ provide essentially complete fluorous phase immobilization $(>99.7:<0.3)$. The hydrogenation of the pyridine in entry VIII-5 to the piperidine in entry VIII-8 slightly increases fluorophilicity (90.4:9.6 vs. 93.6:6.4). This secondary amine can in turn be compared to $\mathrm{HN}\left[\left(\mathrm{CH}_{2}\right)_{5} \mathrm{R}_{\mathrm{f} 8}\right]_{2}$ (entry X-9) which has one less $\mathrm{CH}_{n}$ group and a very similar fluorous phase affinity $(93.0: 7.0)$

\subsection{6}

\section{Metal Complexes}

Section XVI of Table 6-1 features a variety of metal complexes, the majority of which have been engineered to have high fluorophilicities. As illustrated by entries XVI-40 through to XVI-45, a ferrocene appears slightly more difficult to immobilize than a benzene ring. Two ponytails of the formula $\mathrm{R}_{\mathrm{f} 10}\left(\mathrm{CH}_{2}\right)_{2}$ or four ponytails of the formula $\mathrm{R}_{\mathrm{f} 4}\left(\mathrm{CH}_{2}\right)_{2}$ suffice for $\mathrm{CF}_{3} \mathrm{C}_{6} \mathrm{~F}_{11} /$ toluene partition coefficients of $>91:<9$. The fluorous zirconocenes $\left(\eta^{5}\right.$ $\left.\mathrm{C}_{5} \mathrm{H}_{4}\left(\mathrm{CH}_{2}\right)_{2} \mathrm{R}_{\mathrm{f} 6}\right)_{2} \mathrm{ZrX}_{2}$ exhibit $\mathrm{CF}_{3} \mathrm{C}_{6} \mathrm{~F}_{11} /$ toluene partition coefficients of 95.4:4.6 (X= Cl, entry XVI-46) and 87.5:12.5 (X $=\mathrm{CH}_{3}$, entry XVI-47).

Compounds that are catalyst precursors are of particular interest. The rhodium complexes in entries XVI-9 and XVI-10, which feature three phosphine ligands of the formula $\mathrm{P}\left[\left(\mathrm{CH}_{2}\right)_{2} \mathrm{R}_{\mathrm{fn}}\right]_{3}$, exhibit very high fluorophilicities $\left(\mathrm{CF}_{3} \mathrm{C}_{6} \mathrm{~F}_{11} /\right.$ toluene partition coefficients 99.86:0.14, $n=6$, and 99.88:0.12, $n=8$ ). Those of similar square planar iridium and nickel complexes with two such phosphine ligands are slightly lower (entries XVI-30 through to XVI-34). Salts should normally have poor fluorous phase affinities. However, entries XVI-20 and XVI-24 show that when appropriate cationic fluorous rhodium complexes and fluorous anions are combined, $\mathrm{CF}_{3} \mathrm{C}_{6} \mathrm{~F}_{11} /$ toluene partition coefficients of 97.1:2.9 to 98.5:1.5 can be achieved. Although partition coefficients have not yet been reported, polyoxometalate salts with fluorous tetralkylammonium cations can exhibit similar fluorophilicities [11].

Entry XVI-12 illustrates an interesting effect. The central rhodium is surrounded by three fluorous triarylphosphines that have only one ponytail per ring, and a $\mathrm{CF}_{3} \mathrm{C}_{6} \mathrm{~F}_{11} / n-\mathrm{C}_{8} \mathrm{H}_{18}$ partition coefficient of 52:48 at $0{ }^{\circ} \mathrm{C}$ (entry XI-40). Nonetheless, the rhodium complex is highly fluorophilic, with a partition coefficient of 99.7:0.3 under analogous conditions. Similar phenomena, in which the "sum is greater than the parts", have been observed with other compounds that are aggregates of fluorous building blocks. Two effects are probably at work. Firstly, the ponytails are deployed in a maximally efficient way around the periphery of the molecule. Secondly, the molar volume increases, the influence of which is discussed in Section 6.5 below.

Dirhodium tetracarboxylates $\mathrm{Rh}_{2}\left[\mathrm{O}_{2} \mathrm{C}\left(\mathrm{CH}_{2}\right)_{m} \mathrm{R}_{\mathrm{fn}}\right]_{4}$ with very high fluorous phase affinities are also available (entries XVI-1 through to XVI-8), although for some reason $\mathrm{CF}_{3} \mathrm{C}_{6} \mathrm{~F}_{11} /$ ether gives much less biased partition coefficients than $/ \mathrm{CH}_{2} \mathrm{Cl}_{2}, / \mathrm{C}_{6} \mathrm{H}_{14}$, or /toluene mixtures. The fluorous nitrogen and sulfur palladacycles in entries XVI-48 through to XVI-51 
contain three ponytails of the formula $\mathrm{R}_{\mathrm{f} 8}\left(\mathrm{CH}_{2}\right)_{m}(m=2$ or 3$)$ per arene ring. The $\mathrm{CF}_{3} \mathrm{C}_{6} \mathrm{~F}_{11}$ /toluene partition coefficients (95.5:4.5 and 90.7:9.3) indicate fluorophilicities somewhat lower than those of the free non-palladated ligands (98.7:1.3 and 99.5:0.5, entries $\mathrm{X}-24$ and XIV-15).

\section{4}

\section{General Trends and Special Situations}

Table 6-1 shows that, with only a very few exceptions, the introduction of longer ponytails or additional ponytails leads to higher fluorous phase affinities. When additional methylene spacers are introduced, while keeping the perfluoroalkyl segments constant, fluorous phase affinities decrease. In the authors' view, compounds with $\mathrm{CF}_{3} \mathrm{C}_{6} \mathrm{~F}_{11}$ /toluene partition coefficients of $>90:<10$ have high fluorophilicities, and those with partition coefficients of $>99:<1$ possess very high fluorophilicities. When the ratio exceeds $>99.7:<0.3$, the compounds can be viewed as "immobilized".

In general, simple monofunctional organic compounds, including arenes, require two ponytails of the formula $\left(\mathrm{CH}_{2}\right)_{m} \mathrm{R}_{\mathrm{f} 8}(m=2,3)$ for high fluorophilicities. Three such ponytails lead to very high fluorophilicities, and often essentially complete immobilization. An early rule of thumb stated that for a molecule to be preferentially soluble in a fluorous liquid phase (partition coefficient $>50:<50$ ), $60 \%$ of the molecular weight should be fluorinederived [12]. However, Table 6-1 contains exceptions to all of these generalizations, and selected cases are now examined in turn.

In compounds that already contain a long perfluoroalkyl segment, the introduction of a $\mathrm{CF}_{3}$ group or a "pigtail" sometimes imparts a fluorophilicity significantly greater than might be expected. For example, the $\mathrm{R}_{\mathrm{f} 8}$-monosubstituted benzene in entry VII-16 can be compared with the $\mathrm{R}_{\mathrm{f} 8} / \mathrm{CF}_{3}$ - and $\mathrm{R}_{\mathrm{f} 8} / \mathrm{R}_{\mathrm{f8}}$-disubstituted benzenes in entries VII-37 through to VII-40 and the $\mathrm{R}_{\mathrm{f} 8} / \mathrm{CF}_{3} / \mathrm{CF}_{3}$-trisubstituted benzene in entry VII-60. Although the $\mathrm{CF}_{3} \mathrm{C}_{6} \mathrm{~F}_{11} /$ toluene partition coefficients for the $\mathrm{R}_{\mathrm{f} 8} / \mathrm{CF}_{3}$ compounds [91.5:8.5 (meta), 89.4:10.6 (para), 81.8:18.2 (ortho)] indicate fluorophilicities less than that of the $\mathrm{R}_{\mathrm{f8}} / \mathrm{R}_{\mathrm{f8}}$ compound (99.3:0.7), they are in two cases distinctly greater than that of the $\mathrm{R}_{\mathrm{f8}}$-monosubstituted compound (77.5:22.5). The $\mathrm{R}_{\mathrm{f} 8} / \mathrm{CF}_{3} / \mathrm{CF}_{3}$ compound (98.3:1.7) is nearly as fluorophilic as the $\mathrm{R}_{\mathrm{f} 8} / \mathrm{R}_{\mathrm{f} 8}$ compound. Thus, although trifluoromethylbenzene itself has a very poor fluorous phase affinity (entry VII-6, 12.4:87.6), $\mathrm{CF}_{3}$ groups represent legitimate design elements for enhancing fluorophilicities once a ponytail is in place. Other compounds that appear to show similar effects can be found in entries VII-33 through to VII-36 and VII-55 through to VII-59.

A possibly related effect, already noted in Section 6.3.6, is as follows. Some compounds that can be viewed as aggregates of fluorous building blocks give partition coefficients distinctly higher than the individual building blocks. For example, the fluorophilicity of the rhodium tris(phosphine) complex in entry XVI-12 is much greater than that of the phosphine ligand (entry XI-40). Another manifestation of this phenomenon, but in a supramolecular context, is illustrated in Scheme 6-1 [13]. The fluorous $N, N^{\prime}$-dialkyl urea 1 is not very fluorophilic $\left(\mathrm{C}_{6} \mathrm{~F}_{14} / \mathrm{CH}_{2} \mathrm{Cl}_{2}\right.$ partition coefficient 30:70). However, the addition of an equivalent of the fluorous carboxylic acid 2 gives a highly fluorophilic 1:1 complex (partition coefficient 99:1). Although the partition coefficient of 2 is not known, it could well be 


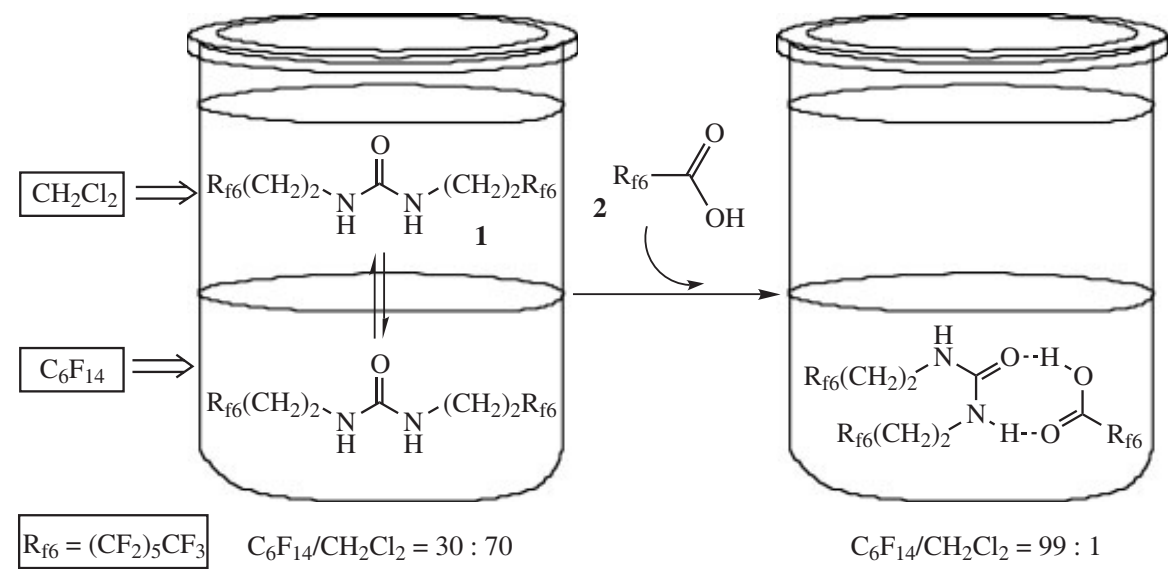

Scheme 6-1. Effect of supramolecular complex formation upon a partition coefficient

somewhat lower due to the polar acidic functional group. In this event, two more polar solutes combine to give a less polar and more fluorophilic complex. Additional examples are provided in entries XVII-1 through to XVII-9 of Table 6-1.

In such compounds or complexes, three main effects are probably operating. Firstly, polar acidic and basic sites (either Brønsted or Lewis) combine to give less polar moieties. Secondly, the ponytails are directed around the outer perimeter of the molecule in an efficient manner such that solvation in non-fluorous media is impeded. One wonders whether a "Maginot line" (or perhaps more accurately, a "Maginot sphere") of $\mathrm{CF}_{3}$-pigtails, accompanied by a smattering of ponytails, might constitute a particularly staunch defender of the fluorous character. Thirdly, the molar volume increases, the effect of which is discussed in the following section.

The most puzzling exceptions to the generalizations regarding ponytail length and quantity and fluorous phase affinities involve silicon-substituted triarylphosphines of the formula $\mathrm{P}\left\{p-\mathrm{C}_{6} \mathrm{H}_{4} \mathrm{Si}\left(\mathrm{CH}_{3}\right)_{3-x}\left[\left(\mathrm{CH}_{2}\right)_{2} \mathrm{R}_{\mathrm{fn}}\right]_{x}\right\}_{3}$ (entries XI-39 through to XI-58). The partition coefficients were carefully measured in several solvent systems [9a]. The values in $\mathrm{CF}_{3} \mathrm{C}_{6} \mathrm{~F}_{11} / n$ pentane show the expected monotonic trend, with fluorophilicities increasing in the order $x / n=1 / 6<1 / 8<2 / 6<2 / 8<3 / 6<3 / 8$. However, in $\mathrm{CF}_{3} \mathrm{C}_{6} \mathrm{~F}_{11} / n$-octane the phosphines with three ponytails show lower fluorous phase affinities than those with two ponytails $(x / n=1 / 6<1 / 8<\underline{3} / 6<\underline{3} / 8<\underline{2} / 6<\underline{2} / 8)$. The situation is similar in $\mathrm{CF}_{3} \mathrm{C}_{6} \mathrm{~F}_{11} /$ toluene, but now with some of compounds with $\mathrm{R}_{\mathrm{f} 6}$ ponytails showing fluorous phase affinities equal to or greater than the homologs with $\mathrm{R}_{\mathrm{f} 8}$ ponytails $(x / n=1 / 6<1 / 8<3 / \underline{8}<$ $3 / \underline{6}<2 / \underline{8}=2 / \underline{6})$.

The preceding trends cannot be rationalized by any of the qualitative fluorophilicity models, but yet are real and must have an explanation. Apart from questioning the models, it should be noted that for measurements made in solution, there is always the possibility that certain fluorous solutes, but not others, might aggregate in some way. Gel formation is not uncommon, and micelles are certainly conceivable. As noted in Section 6.2, in the most 
Tab. 6-2. Effect of fluorous solvent on partition coefficients with toluene $\mathrm{e}^{\mathrm{a}, \mathrm{b}}$

\begin{tabular}{|c|c|c|}
\hline Solvent & $\mathrm{CH}_{3} \mathrm{CH}_{2} \mathrm{CH}\left(\mathrm{CH}_{3}\right) \mathrm{CO}_{2}\left(\mathrm{CH}_{2}\right)_{2} R_{f 8}$ & $\mathrm{CH}_{3}\left(\mathrm{CH}_{2}\right)_{2} \mathrm{CH}\left(\mathrm{CH}_{3}\right) \mathrm{CO}_{2}\left(\mathrm{CH}_{2}\right)_{7} R_{f 8}$ \\
\hline \multirow[t]{2}{*}{$\left(\mathrm{CF}_{3}\right)_{3} \mathrm{CC}\left(\mathrm{CF}_{3}\right)_{3} \mathrm{c}$} & $39: 61$ & $32: 68$ \\
\hline & $(0.64)$ & $(0.48)$ \\
\hline \multirow[t]{2}{*}{$n-\mathrm{C}_{6} \mathrm{~F}_{14}$} & $50: 50$ & $40: 60$ \\
\hline & $(0.98)$ & $(0.68)$ \\
\hline \multirow[t]{2}{*}{$\mathrm{CF}_{3} \mathrm{C}_{6} \mathrm{~F}_{11}$} & $55: 45$ & $49: 51$ \\
\hline & $(1.23)$ & $(0.95)$ \\
\hline \multirow[t]{2}{*}{ perfluoroperhydrofluorene ${ }^{\mathrm{d}}$} & $53: 47$ & $49: 51$ \\
\hline & $(1.13)$ & $(0.97)$ \\
\hline \multirow[t]{2}{*}{ perfluorodecalin ${ }^{\mathrm{d}}$} & $61: 39$ & $56: 44$ \\
\hline & $(1.59)$ & $(1.28)$ \\
\hline
\end{tabular}

a Fluorous solvent:toluene ( $P$ value as originally determined). ${ }^{\mathrm{b}} 25^{\circ} \mathrm{C}$ unless noted. ${ }^{c} 40^{\circ} \mathrm{C}$. d Mixtures of cis/trans isomers.

rigorous studies, the concentration dependences of partition coefficients are determined. The data are then extrapolated to infinite dilution, where aggregates become impossible. Perhaps some of these non-monotonic trends reflect non-ideal solution behavior.

For many compounds in Table 6-1, partition coefficients were measured with more than one non-fluorous solvent. Although there are a few curious exceptions (e.g., ether vs. toluene or hexane in entries XVI-1 through to XVI-8), the more polar the non-fluorous solvent, the greater the fraction of the fluorous solute in the fluorous phase.

In contrast, only a few compounds have been probed with more than one fluorous solvent. Naturally, it is of interest to know which ones give the most biased partition coefficients. This has been investigated with two test solutes and toluene as summarized in Table 6-2 [14]. The best results were obtained with perfluorodecalin, and all cyclic solvents tested were superior to $n-\mathrm{C}_{6} \mathrm{~F}_{14}$. Entries of VI-8 through to VI-107 of Table 6-1 also feature many parallel measurements involving $\mathrm{CF}_{3} \mathrm{C}_{6} \mathrm{~F}_{11}$ and $n-\mathrm{C}_{6} \mathrm{~F}_{14}$. In nearly all cases, the former gives the more biased partition coefficient.

\section{5}

\section{Quantitative Analysis and Prediction of Partition Coefficients}

There have been several efforts to parameterize the above data such that fluorophilicities can be predicted [4-6]. One approach makes use of 3D QSAR descriptors and neural networks [4]. A host of parameters was considered: percent fluorine, molecular volume, molecular surface, globularity, solvent accessible surface, solvent extended surface, solvent extended volume, calculated polarizability, calculated dipole moment, calculated Hildebrand parameter, degree of chemical bond rotational freedom, and others. In the end, very good agreement between measured and predicted partition coefficients in the test group of 60 molecules was realized.

Another parameterization effort involving a test group of 90 molecules found fluorine content, dispersion, and hydrogen bond acidity factors to be most important [4]. Polarity, hydrogen bond basicity, and size effects played much smaller roles. 
The most definitive work to date involves Mobile Order and Disorder (MOD) theory [6, 15]. This has been applied to other types of liquid/liquid partition coefficients, using a fiveterm expression. With aprotic fluorous biphasic systems, the expression reduces to two terms. One involves the molar volumes of the fluorous and organic solvents and the solute. The other involves the corresponding cohesion parameters. Once the solvent properties have been measured, the partition coefficients are a function of only two solute variables, one of which (the molar volume) is easily calculated from group increments. The investigators use a small amount of the data in Table 6-1 to back-calculate the solute cohesion parameter and derive a second set of group increments. This allows the partition coefficients of approximately 50 additional compounds to be predicted with good accuracy.

\section{6}

\section{Future Directions}

On the computational side, the prediction of partition coefficients is certain to attract further attention. To better interpret raw data, fluorous solutes will be increasingly scrutinized for non-ideal behavior in solution. Naturally, there will be many new entries for Table 6-1, as well as increased focus on supramolecular assemblies. However, there are some likely directions for future research that are not straightforward extensions of themes discussed above.

For example, partition coefficients for fluorous liquid/non-fluorous solid biphase systems are of interest from several standpoints. Recently, the partitioning of small organic molecules from both fluorous and non-fluorous solvents as well as mixtures into highly crosslinked, macroporous, and insoluble organic polymers has been studied [16]. One goal is to enhance access of organic substrates to imbedded catalyst sites. Presumably due to a fluorophobic effect, fluorous/organic solvent mixtures give up to 200-fold increases in local concentrations relative to pure organic solvents. Under catalytic conditions, turnover frequencies are greatly enhanced.

6.7

\section{Sample Experimental Determinations}

The following procedures illustrate recommended procedures for determining partition coefficients by GLC, HPLC, and ${ }^{19} \mathrm{~F}$ NMR. All correspond to entries in Table 6-1.

A [17]. A $10 \mathrm{~mL}$ vial was charged with 3,4-( $\left(\mathrm{R}_{88} \mathrm{CH}_{2} \mathrm{CH}_{2} \mathrm{CH}_{2}\right)_{2} \mathrm{C}_{6} \mathrm{H}_{3} \mathrm{I}$ (entry VII-50; $0.0156 \mathrm{~g}, 0.0138 \mathrm{mmol}), \mathrm{CF}_{3} \mathrm{C}_{6} \mathrm{~F}_{11}(2.000 \mathrm{~mL})$, and $\mathrm{MeOH}(2.000 \mathrm{~mL})$, fitted with a mininert valve, vigorously shaken $(2 \mathrm{~min})$, and immersed (cap-level) in a $35{ }^{\circ} \mathrm{C}$ oil bath. After $12 \mathrm{~h}$, the bath was removed. After $12-24 \mathrm{~h}$, a $0.500 \mathrm{~mL}$ aliquot of each layer was added to $0.250 \mathrm{~mL}$ of a standard $0.0244 \mathrm{M}$ solution of eicosane in hexane. The samples were diluted with ether and GLC analysis (average of 7-8 injections) showed that $0.00325 \mathrm{mmol}$ of 3,4$\left(\mathrm{R}_{\mathrm{f} 8} \mathrm{CH}_{2} \mathrm{CH}_{2} \mathrm{CH}_{2}\right)_{2} \mathrm{C}_{6} \mathrm{H}_{3} \mathrm{I}$ was in the $\mathrm{CF}_{3} \mathrm{C}_{6} \mathrm{~F}_{11}$ aliquot and $0.000101 \mathrm{mmol}$ in the $\mathrm{MeOH}$ aliquot (97.0:3.0; a 2.000/0.500 scale factor gives a total mass recovery of $0.0150 \mathrm{~g}, 97 \%$ ).

B [18]. A $10 \mathrm{~mL}$ vial was charged with the imine palladacycle from entry XVI-48; $(0.0104 \mathrm{~g}$, $0.0031 \mathrm{mmol}), \mathrm{CF}_{3} \mathrm{C}_{6} \mathrm{~F}_{11}(2.000 \mathrm{~mL})$, and toluene $(2.000 \mathrm{~mL})$, fitted with a mininert valve, 
and vigorously shaken $(2 \mathrm{~min})$. After $2 \mathrm{~h}\left(24^{\circ} \mathrm{C}\right)$, a $0.250 \mathrm{~mL}$ aliquot of the fluorous phase and a $0.750 \mathrm{~mL}$ aliquot of the non-fluorous phase were removed. The solvents were evaporated and the residues dried by oil pump vacuum $(1 \mathrm{~h})$. Each residue was taken up in $\mathrm{CF}_{3} \mathrm{C}_{6} \mathrm{H}_{5} / \mathrm{EtOH}(9: 1 \mathrm{v} / \mathrm{v} ; 0.500 \mathrm{~mL})$ and analyzed by HPLC (average of 5 injections, $200 \times 4$ mm Nucleosil 100-5 column, UV/visible detector). The relative peak intensities were (after normalization to the aliquot volumes) $95.5: 4.5$.

C [19]. A $5 \mathrm{~mL}$ flask was charged with $\mathrm{H}_{3} \mathrm{~B} \cdot \mathrm{P}\left[p-\mathrm{C}_{6} \mathrm{H}_{4}\left(\mathrm{CH}_{2}\right)_{3} \mathrm{CH}\left(\mathrm{CH}_{2} \mathrm{R}_{\mathrm{f} 8}\right)_{2}\right]_{3}$ (entry XI-21; $0.0368 \mathrm{~g}, 0.0121 \mathrm{mmol})$ and $\mathrm{CF}_{3} \mathrm{C}_{6} \mathrm{~F}_{11}(2.00 \mathrm{~mL})$. After complete dissolution, toluene $(2.00$ $\mathrm{mL}$ ) was added and the mixture was vigorously shaken $(20 \mathrm{~min})$. The flask was kept at $25{ }^{\circ} \mathrm{C}$ for $48 \mathrm{~h}$. Then aliquots (each of $0.500 \mathrm{~mL}$ ) were taken from both phases. The $\mathrm{CF}_{3} \mathrm{C}_{6} \mathrm{~F}_{11}$ aliquot was evaporated to dryness. A solution of the internal standard $\mathrm{C}_{6} \mathrm{~F}_{6}(0.0738 \mathrm{~g}$, $0.397 \mathrm{mmol}$ ) in $\mathrm{CF}_{3} \mathrm{C}_{6} \mathrm{H}_{5}$ (12.7016 g) was prepared. Portions of this standard solution were added gravimetrically to the above aliquots $\left(\mathrm{CF}_{3} \mathrm{C}_{6} \mathrm{~F}_{11}\right.$ : $0.6413 \mathrm{~g}$ solution, $0.0199 \mathrm{mmol}$ $\mathrm{C}_{6} \mathrm{~F}_{6}$; toluene: $0.0596 \mathrm{~g}$ solution, $\left.0.00185 \mathrm{mmol} \mathrm{C}_{6} \mathrm{~F}_{6}\right)$. Then $\mathrm{C}_{6} \mathrm{D}_{6}$ was added $(0.05 \mathrm{~mL}$ each $)$ and the samples were analyzed by ${ }^{19} \mathrm{~F}$ NMR (integration of $\mathrm{CF}_{3}$ signal against $\mathrm{C}_{6} \mathrm{~F}_{6}$ ). The procedure was repeated, giving an average partition coefficient of 96.6:3.4 \{0.00840 $\mathrm{g}$ of $\mathrm{H}_{3} \mathrm{~B} \cdot \mathrm{P}\left[p-\mathrm{C}_{6} \mathrm{H}_{4}\left(\mathrm{CH}_{2}\right)_{3} \mathrm{CH}\left(\mathrm{CH}_{2} \mathrm{R}_{\mathrm{f} 8}\right)_{2}\right]_{3}$ in $0.500 \mathrm{~mL}$ of $\mathrm{CF}_{3} \mathrm{C}_{6} \mathrm{~F}_{11} ; 0.000292 \mathrm{~g}$ of $\mathrm{H} 3 \cdot \mathrm{P}[p-$ $\left.\mathrm{C}_{6} \mathrm{H}_{4}\left(\mathrm{CH}_{2}\right)_{3} \mathrm{CH}\left(\mathrm{CH}_{2} \mathrm{R}_{\mathrm{f} 8}\right)_{2}\right]_{3}$ in $0.500 \mathrm{~mL}$ of toluene\}. A $2.00 / 0.500$ scale factor gives a total mass recovery of $0.0348 \mathrm{~g}(95 \%)$.

\section{References}

1 Leo, A.; Hansch, C.; Elkins, D. Chem. Rev. 1971, 71, 525.

2 Solé-Violan, L.; Devallez, B.; Postel, M.; Riess, J. G. New. J. Chem. 1993, 17, 581.

3 Barthel-Rosa, L. P.; Gladysz, J. A. Coord. Chem. Rev. 1999, 190-192, 587.

4 Kiss, L. E.; Kövesdi, I.; RÁbaI, J. J. Fluorine Chem. 2001, 108, 95.

5 Huque, F. T. T.; Jones, K.; Saunders, R. A.; Platts, J. A. J. Fluorine Chem. 2002, 115, 119.

6 (a) De Wolf, E.; Ruelle, P.; van deN Broeke, J.; Deelman, B.-J.; van Koten, G. manuscript in preparation. (b) DE Wolf, E. Doctoral Thesis, University of Utrecht, 2002, Chapter 7.

7 It should be emphasized in passing that an appreciable amount of the very polar solute methanol can be found in the fluorous phase of $\mathrm{CF}_{3} \mathrm{C}_{6} \mathrm{~F}_{11} /$ toluene mixtures. However, the authors are not aware of any quantitative measurements. This dramatically illustrates the effect of the sizes of non-fluorous solutes on solubilities and partition coefficients.
8 Curran, D. P.; Hadida, S.; Kim, S.-Y.; Luo, Z. J. Am. Chem. Soc. 1999, 121, 6607.

9 (a) Richter, B.; De Wolf, E.; van Koten, G.; Deelman, B.-J. J. Org. Chem. 2000, 65, 3885. (b) DE Wolf, E.; Richter, B.; Deelman, B.-J.; van Koten, G. J. Org. Chem. 2000, 65, 5424. (c) Richter, B.; Spek, A. L.; van Koten, G.; Deelman, B.-J. J. Am. Chem. Soc. 2000, 122, 3945. (d) De Wolf, E.; Speets, E. A.; Deflman, B.-J.; van Koten, G. Organometallics 2001, 20, 3686

10 For a parallel approach to fluorous benzyl ethers, see Curran, D. P.; Ferritto, R.; HuA, Y. Tetrahedron Lett. 1998, 39, 4937.

11 Maayan, G.; Fish, R. H.; Neumann, R. Org. Lett. 2003, 5, 3547.

12 Horváth, I. T. Acc. Chem. Res. 1998, 31, 641.

13 Palomo, C.; Aizpurua, J. M.; Loinaz, I.; FernandeZ-Berridi, M. J.; Irusta, L. Org. Lett. 2001, 3, 2361.

14 Beier, P.; Cole-Hamilton, D., unpublished data, University of St. Andrews. 
$100 \mid 6$ Partition Coefficients Involving Fluorous Solvents

15 (a) Ruelle, P.; Rey-Mermet, C.;

Buchmann, M.; Nam-Tran, H.;

Kesselring, U. W.; Huyskens, P. L.

Pharmaceut. Res. 1991, 8, 840. (b) Ruelle,

P.; Farina-Cuendet, A.; Kesselring,

U. W. Perspectives Drug Discov. Design 2000, 18, 61. (c) Ruelle, P. J. Chem. Inf. Comput. Sci. 2000, 18, 61.
16 Leeder, S. M.; Gagné, M. R. J. Am. Chem. Soc. 2003, 125, 9048.

17 Rocaboy, C.; Gladysz, J. A. Chem. Eur. J. 2003, 9, 88.

18 Rocaboy, C.; Gladysz, J. A. New J. Chem. 2003, 27, 39.

19 Wende, M.; Seidel, F.; Gladysz, J. A. J. Fluorine Chem. 2003, 124, 45. 